\title{
Aportes al conocimiento de los Laniatores (Arachnida: Opiliones: Grassato- res) del departamento de Loreto, Perú
}

\author{
Contributions to knowledge of Laniatores (Arachnida: Opiliones: Grassatores) of the de- \\ partment of Loreto, Peru
}

\section{Arli Ayala-Apaza* \\ https://orcid.org/0000-0001-8496-7769 \\ arlibertad@gmail.com \\ Diana Silva-Dávila \\ https://orcid.org/0000-0002-7371-099X \\ diana.silva@unmsm.edu.pe \\ *Corresponding author \\ Universidad Nacional Mayor de San Marcos, Museo de Historia Natural, Departamento de Entomología. Av. Arenales 1256, Jesús María 15072, Lima-Perú. \\ Citación \\ Ayala-Apaza A, Silva-Dávila D. 2021. Aportes al \\ conocimiento de los Laniatores (Arachnida: Opiliones: Grassatores) del departamento de Loreto, Perú. Revista peruana de biología 28(especial): e20695 001- 022 (Diciembre 2021). doi: http://dx.doi.org/10.15381/rpb v28iespecial.20695}

\section{Presentado: $\quad 09 / 02 / 2021$ \\ Aceptado: $\quad 10 / 08 / 2021$ \\ Publicado online: 30/12/2021}

Editor:

Leonardo Romero

\section{Resumen}

En este trabajo presentamos un análisis del estado del conocimiento y riqueza de los opiliones Laniatores del departamento de Loreto; en general, arácnidos poco conocidos en la Amazonia peruana. Para ello, se revisó la literatura relevante y se examinó la colección del Museo de Historia Natural de la Universidad Nacional Mayor de San Marcos, Lima, Perú. Los resultados muestran 72 especies distribuidas en 11 familias. Las familias más diversas fueron Cosmetidae (34 spp.), Cranaidae (11 spp.) y Stygnidae (8 spp.). Aunque no hay información suficiente, las muestras del río Samiria (23 especies, 11 familias) sugieren un grado de endemismo local muy alto, 10 especies están restringidas solo a esta localidad. Con este trabajo se suman 52 especies de Laniatores a las 19 previamente registradas en la literatura para el departamento de Loreto y se registran por primera vez en Perú las familias Biantidae, Samoidae y Stygnommatidae. Aquí se reportan 12 por primera vez para Perú: Cocholla simoni Roewer, Cynorta marginalis Banks, Discosomaticus cinctus (Perty), Meterginus serratus Roewer, Paecilaemula argentinoi Soares, Sibambea rotunda Roewer, Taito insperatus Kury \& Barros, Taito rorschachi Kury \& Barros, Ventripila marginata Roewer, Hutamaia plei Tourinho \& Mendes, Obidosus boibumba (Villarreal \& Pinto-da-Rocha), y Stenostygnus pusio Simon.

\section{Abstract}

In this paper we present an analysis of the state of knowledge and species richness of the Laniatores harvestmen from the department of Loreto, a group of little-known arachnids in the Peruvian Amazon. To do this, relevant literature was reviewed and the collection of the Natural History Museum of the Universidad Nacional Mayor de San Marcos, Lima, Peru was examined. Our results show 72 species distributed in 11 families. Cosmetidae (34 spp.), Cranaidae (11 spp.) and Stygnidae (8 spp.) were the most diverse families. Although there is insufficient information, samples from Samiria River (23 species, 11 families) suggest a very high degree of local endemism, 10 species are restricted only to this locality. With this work, 52 species of Laniatores are added to 19 previously recorded for the Loreto department. Families Biantidae, Samoidae and Stygnommatidae are recorded for the first time in Peru. In addition, 12 new species are reported here for the first time in Peru: Cocholla simoni Roewer, Cynorta marginalis Banks, Discosomaticus cinctus (Perty), Meterginus serratus Roewer, Paecilaemula argentinoi Soares, Sibambea rotunda Roewer, Taito insperatus Kury \& Barros, Taito rorschachi Kury \& Barros, Ventripila marginata Roewer, Hutamaia plei Tourinho \& Mendes, Obidosus boibumba (Villarreal \& Pintoda-Rocha), and Stenostygnus pusio Simon.

Palabras clave:

Biantidae; Samoidae; Stygnommatidae; río Samiria; Amazonia peruana.

Keywords:

Biantidae; Samoidae; Stygnommatidae; Samiria River; Peruvian Amazon. 


\section{Introducción}

La clase Arachnida son un grupo muy diverso de artrópodos que comprenden aproximadamente 570 familias, de las cuales el 25\% ocurren en la Amazonia (Adis 2002); dentro de este grupo, los opiliones constituyen el cuarto orden más diverso de la clase con 6637 especies válidas en todo el mundo (Kury et al. 2020). Excluyendo los fósiles, se reconocen cuatro subórdenes: Cyphophthalmi, Eupnoi, Dyspnoi y Laniatores, siendo este último el más diverso con 39 familias y 4186 especies (Kury et al. 2020).

Los opiliones se destacan por su gran diversidad morfológica y la variedad de su repertorío de comportamiento, principalmente en los trópicos y en particular en los bosques húmedos. La mayoría de las especies son depredadoras, pero también hay algunas que son omnívoras o especializadas en el consumo de restos de plantas, hongos, algas, etc. (Curtis \& Machado 2007, Acosta \& Machado 2007). Sus preferencias de hábitat son amplias y cubren diferentes estratos, desde el suelo, hojarasca y arbustos hasta el dosel arbóreo; algunas especies incluso pueden tener preferencias muy específicas como cuevas o nidos de hormigas (Curtis \& Machado 2007).

Brasil es el país con la mayor diversidad de opiliones en el mundo y de las, aproximadamente, 1000 especies conocidas, 855 corresponden a Laniatores (Villarreal et al. 2021, 2003a); por estudios recientes, Venezuela con 392 especies es el segundo país más diverso (Villarreal et al. 2021), esta diferencia en el número de especies descritas refleja el gran esfuerzo taxonómico que se requiere para conocer la opiliofauna de Sudamérica y esclarecer sus patrones de distribución. Por otro lado, los opiliones han sido utilizados como modelos para análisis de endemismo (v.g. DaSilva et al. 2015) debido a su escasa capacidad de dispersión (Giribet \& Kury 2007) resaltando el alto grado de endemismo regional encontrado.

Kury $(2020,2003 a)$ registra 178 especies para Perú, de las cuales 159 son Laniatores y se observa un alto grado de endemismo considerando que, de todas las especies registradas, 166 se conocen solo de Perú. Los trabajos más antiguos que reportan opiliones en este país corresponden a descripciones taxonómicas (e.g. Chamberlin 1916, Mello-Leitão 1949, Roewer 1957, Soares \& Bauab-Vianna 1973); en tanto que trabajos más contemporáneos están enfocados en esclarecer las relaciones filogenéticas de algunos grupos, como por ejemplo, Pinto-da-Rocha (1997) quien en su revisión sistemática de la familia Stygnidae, actualiza el estado taxonómico de algunas especies peruanas, y Pinto-da-Rocha y Bragagnolo (2017), quienes estudiaron las relaciones filogenéticas de la familia Nomoclastidae, y como resultado parcial describen una nueva especie: Callcosma cofan Pinto-da-Rocha \& Bragagnolo, 2017, conocida de Perú (Loreto) y Ecuador.

En la literatura solo se han registrado 19 especies de Laniatores para el departamento de Loreto, por lo que en este trabajo se proporcionan datos actualizados sobre la diversidad y riqueza de estos opiliones amazónicos basados en la revisión de material proveniente de la colección científica del Museo de Historia Natural de la Universidad Nacional Mayor de San Marcos (MUSM) y la compilación de toda la información existente en la literatura especializada. Se espera que esta información sirva de base y oriente la gestión y conservación de la biodiversidad en Loreto.

\section{Material y métodos}

Obtención de registros . - Los registros de Laniatores aquí presentes provienen de datos tomados directamente de las etiquetas de especímenes depositados en la Colección de Arácnidos del Museo de Historia Natural de la Universidad Nacional Mayor de San Marcos (MUSM). El material estudiado (Tabla 1, Anexo 1) proviene de diferentes tipos de muestreos, realizados con propósitos particulares como proyectos de investigación científica, evaluaciones de impacto ambiental (EIA), y recolectas al azar no enfocadas en opiliones.

Tabla 1. Localidades con registros de opiliones Laniatores en el departamento de Loreto en la literatura y este trabajo. Los números representan el total de especies entre este trabajo y los previamente publicados y aquello entre paréntesis, las identificadas con una especie descrita o de registros de especies en la literatura.

\begin{tabular}{llc}
\hline Provincia & Localidad & Especies \\
\hline & Aguas Negras & 3 \\
& Iquitos & 5 \\
\hline & Río Curaray & 20 \\
\hline Máo Itaya & 8 \\
\hline & Río Napo & 26 \\
\hline & Río Nashiño & 7 \\
\hline Río Tahuayo & 1 \\
\hline Loreto 27 (9) & Río Copalyacu & 2 \\
\hline Requena 15 (13) & Río Pacayacu & 1 \\
\hline Río Samiria & 23 \\
\hline Alto amazonas 3 (2) & Trompeteros & 2 \\
\hline Mariscal ramón castilla 8 (8) & Jenaro Herrera & 15 \\
\hline Dátem del Marañón 2 (2) & Pebas & 1 \\
\hline Ucayali 2 (2) & Valle del Marañón & 3 \\
\hline & Bajo Inahuaillo & 2 \\
\hline
\end{tabular}

Otra fuente de registros proviene de la revisión de las publicaciones donde se citan Laniatores para Loreto (Tabla 2) compendiadas en el proyecto OmniPaper (Kury 2003b) y cuya información fue actualizada siguiendo a Kury et al. (2020).

Para la identificación en el laboratorío, se emplearon solo los individuos adultos siguiendo los estándares para la identificación taxonómica (Acosta et al. 2007) ya que las estructuras sexuales secundarias, que se desarrollan por completo en este estadio, proporcionan caracteres 
taxonómicos, especialmente en los machos; sin embargo, para algunas especies, se puede utilizar también el patrón de ornamentación a nivel de género/especie, como en la familia Cosmetidae (v. g., Kury \& Barros 2014).

La identificación para la gran mayoría de taxones quedó a nivel de morfoespecies (Tabla 3), que aquí se definen como unidades con características morfológicas externas únicas para ellas pero que requieren un mayor análisis para corroborar su identidad como especie; a cada una de estas morfoespecies se le asignó un código MUSM único, según el evento de colecta (Anexo 1).

Georeferenciación. - La información de localidades se tomó de las etiquetas de los especímenes examinados, para etiquetas sin datos de coordenadas se usó Google Earth Pro@ v. 7.3.3.7786 para obtener datos aproximados de latitud y longitud; los mapas de distribución fueron elaborados usando grados decimales. Los lugares con registros de Laniatores en el departamento de Loreto se agruparon en 18 localidades tomando como referencia ríos o poblados más conocidos (Fig. 1); una de ellas, Bajo Inahuaillo no tenía registro de coordenadas, pero se sabe que estaba cerca al poblado de Orellana, en la provincia de Ucayali, departamento de Loreto (R. Fernández, com. pers., colector de la muestra), por lo cual la georreferenciación se dio al pueblo de Orellana (Guizado \& Girard 1966). La localidad de Río Pacayacu en la etiqueta se lee como "Pucayacu", pero sus coordenadas la ubican en realidad en el primero. La localidad de Colonia Callería, río Callería, citada previamente dentro de Loreto por Kury (2003b), no fue incluida en este análisis por tratarse en realidad del departamento de Ucayali.

Imágenes. - Para las imágenes, se tomaron fotografías utilizando una cámara integrada a un estereoscopio Nikon SMX745T y la cámara MC-140 acoplada a un estereomicroscopio Leica SAPO. Las secuencias de fotos fueron apiladas en el programa Zerene Stacker@ v. 1.04 y luego procesadas con Adobe Photoshop(C) CC v. 19.0. Los

Tabla 2. Registros de opiliones Laniatores para el departamento de Loreto en la literatura.

\begin{tabular}{|c|c|c|c|}
\hline Familia & Especie & Distribución & Fuente \\
\hline \multirow{8}{*}{ Cosmetidae } & Cynorta cancellata Roewer, 1947 & Perú (Loreto) & Roewer 1947, Kury 2003a \\
\hline & Metacynorta bimaculata Roewer, 1947 & Perú (Loreto) & Roewer 1947, Kury 2003a \\
\hline & Paecilaema guttigerum Sørensen, 1932 & Perú (Loreto) & Kury 2003a \\
\hline & Paecilaemella multimaculata (Wood, 1869) & $\begin{array}{l}\text { Brasil (Acre, Amazonas), } \\
\text { Perú (Loreto) }\end{array}$ & Medrano et al. 2020 \\
\hline & Paecilaema vittatum (Sørensen, 1932) & Perú (Loreto) & Kury 2003a \\
\hline & Pebasia singularis Roewer, 1947 & Perú (Loreto) & Kury 2003a \\
\hline & Poecilaemula peruviana Roewer, 1947 & Perú (Loreto) & Kury 2003a \\
\hline & Taito mayoruna Pinzón M., Damron \& Pinto-da-Rocha, 2021 & Perú (Loreto) & $\begin{array}{l}\text { Pinzón-M, Damron \& Pinto-da-Rocha, } \\
2021\end{array}$ \\
\hline \multirow{4}{*}{ Cranaidae } & Iquitosa poecilis (Roewer, 1943) & $\begin{array}{l}\text { Perú (Loreto, Cusco, San } \\
\text { Martín, Pasco, Huánuco) }\end{array}$ & Kury 2003a, Hara et al. 2014 \\
\hline & Phareicranaus albigyratus Roewer, 1932 & $\begin{array}{l}\text { Colombia (Cundinamar- } \\
\text { ca), Perú (Loreto) }\end{array}$ & $\begin{array}{l}\text { Kury 2003b, Pinto-da-Rocha \& Bonaldo } \\
2011\end{array}$ \\
\hline & Phareicranaus hermosa (Pinto-da-Rocha \& Kury, 2003) & $\begin{array}{l}\text { Ecuador (Napo), Perú } \\
\text { (Loreto) }\end{array}$ & $\begin{array}{l}\text { Pinto-da-Rocha \& Kury 2003, Pinto-da- } \\
\text {-Rocha \& Bonaldo } 2011\end{array}$ \\
\hline & Phareicranaus singularis (Pinto-da-Rocha \& Kury, 2003) & $\begin{array}{l}\text { Brasil (Amazonas), Co- } \\
\text { lombia (Putumayo), Perú } \\
\text { (Loreto) }\end{array}$ & Pinto-da-Rocha \& Kury 2003 \\
\hline Gonyleptidae & Sibollus margaritatus Roewer, 1929 & Perú (Loreto) & $\begin{array}{l}\text { Kury 2003a, Kury \& Alonso-Zarazaga } \\
2011\end{array}$ \\
\hline Manaosbiidae & Tegyra cinnamomea Sørensen, 1932 & Perú (Loreto) & Kury 2003a \\
\hline Nomoclastidae & Callcosma cofan Pinto-da-Rocha \& Bragagnolo, 2017 & $\begin{array}{l}\text { Ecuador (Sucumbíos), } \\
\text { Perú (Loreto) }\end{array}$ & Pinto-da-Rocha \& Bragagnolo 2017 \\
\hline \multirow{4}{*}{ Stygnidae } & Eutimesius simoni Roewer, 1913 & $\begin{array}{l}\text { Brasil (Amazonas), } \\
\text { Colombia (Putumayo), } \\
\text { Ecuador (Los Ríos, Napo), } \\
\text { Perú (Loreto) }\end{array}$ & Kury 2003a \\
\hline & Stygnus klugi (Goodnight \& Goodnight, 1943) & $\begin{array}{l}\text { Perú (Amazonas, Loreto, } \\
\text { Huánuco) }\end{array}$ & Kury 2003a \\
\hline & Stygnus simonis (Sørensen, 1932) & Perú (Loreto) & Kury 2003a \\
\hline & Obidosus albilineatus Roewer, 1957 & $\begin{array}{l}\text { Brasil (Amazonas), Ecua- } \\
\text { dor (Napo), Perú (Loreto, } \\
\text { Madre de Dios, Ucayali) }\end{array}$ & $\begin{array}{l}\text { Ochoa \& Pinto-da-Rocha 2013, Villa- } \\
\text { rreal et al. } 2019\end{array}$ \\
\hline
\end{tabular}




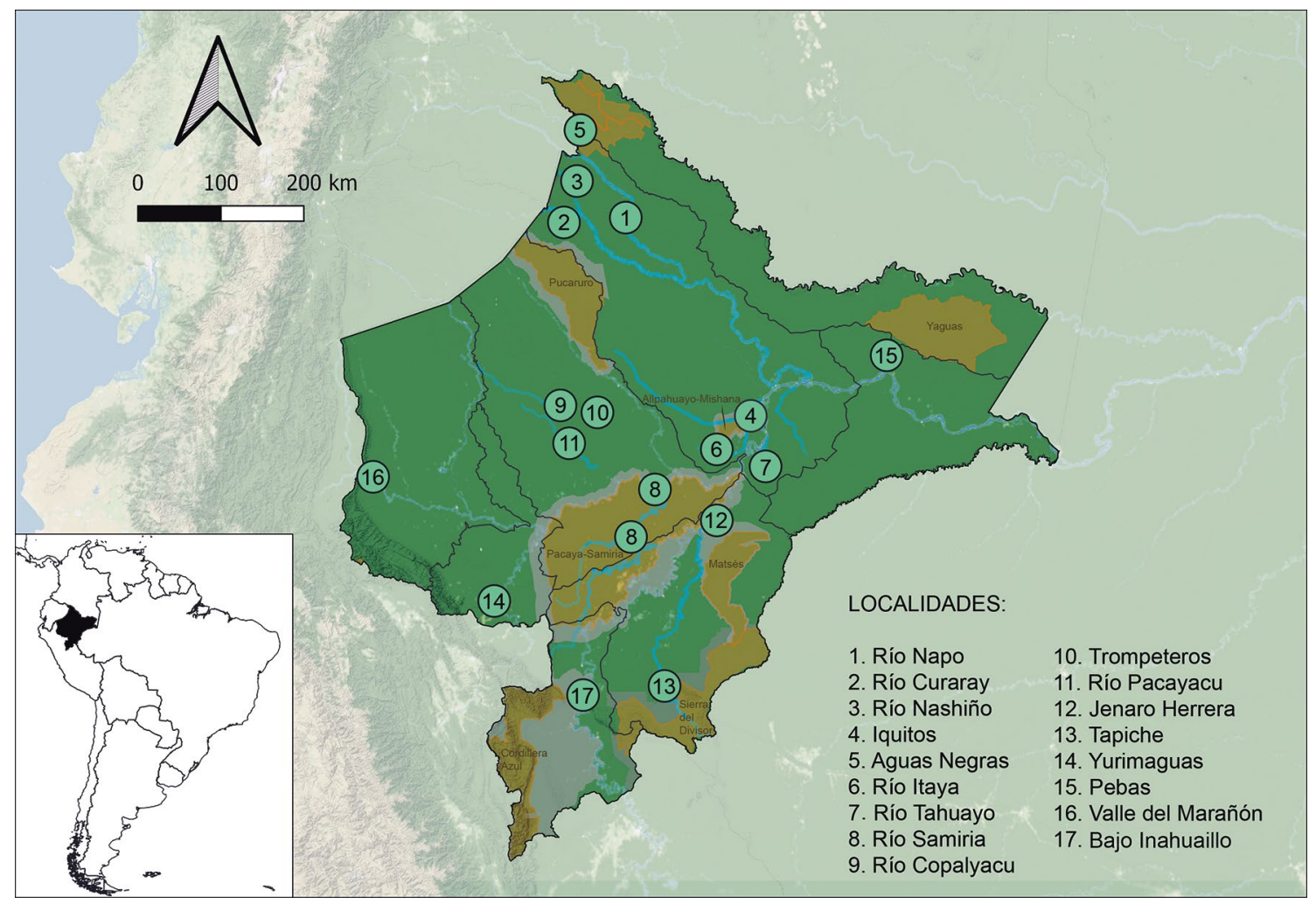

Figura. 1. Localidades con registros de opiliones Laniatores en el departamento de Loreto.

mapas de distribución de algunas de las especies identificadas se realizaron con el programa QGIS ProjectC v. 3.12.2.

Análisis de la riqueza y distribución de especies. - Tomando en cuenta la escasez de datos y la marcada diferencia en el esfuerzo de muestreo para la obtención de las muestras examinadas, la riqueza de especies fue determinada solo por el número de especies presentes en cada localidad (Southwood et al. 1982).

La distribución para la gran mayoría de especies refleja solo los puntos de ocurrencia con base en las etiquetas y los registros en la literatura.

Los especímenes procedentes del río Samiria fueron recolectados mediante búsqueda directa diurna y nocturna, golpeo de vegetación y, también, nebulización de especies arbóreas con diferentes arquitecturas, con copas aisladas o entremezcladas, pudiendo alcanzar los 50 - 55 m de altura (Silva 1996) por lo que usamos esta localidad como punto de referencia para extrapolaciones sobre la riqueza y distribución de especies.

\section{Resultados}

Riqueza y composición de especies. - Los resultados muestran un total de 72 especies para el departamento de Loreto, de las cuales 31 (43\%) están ya identificadas en la literatura (Tablas 2). Las especies examinadas en la colección MUSM (Tabla 3) pertenecen a 11 familias de Laniatores; sin embargo, tres especies no pudieron ser identificadas a nivel de familia por lo que dos de ellas fueron asignadas a Gonyleptoidea y una a Samooidea. Las familias Biantidae, Samoidae y Stygnommatidae son nuevos registros para Perú (Tabla 3, Fig. 2, Anexo 1). Para la familia Cranaidae, se registró un género nuevo para la ciencia proveniente de la localidad de $\mathrm{Cu}$ raray (provincia de Maynas), representado por un macho adulto (MUSM-ENT 0514506) (Tabla 3, Anexo 1). Se halló una especie nueva para la ciencia del género Taito (Cosmetidae) representada por 13 adultos provenientes del río Samiria (Anexo 1) y otra de Thaumatocranaus (Gonyleptidae) representada por seis ejemplares de las localidades de río Napo y río Curaray, ambos en la provincia de Maynas (Anexo 1). Aquí se reportan 12 nuevos registros de especies por primera vez para Perú (Tabla 3, Anexo 1): Cocholla simoni Roewer, 1928 (Fig.3f); Cynorta marginalis Banks, 1909; Discosomaticus cinctus (Perty, 1833); Meterginus serratus Roewer, 1912 (Fig.4c,d); Paecilaemula argentinoi Soares, 1970 (Fig.4e,f); Sibambea rotunda Roewer, 1917 (Fig.3a); Taito insperatus Kury \& Barros, 2014; Taito rorschachi Kury \& Barros, 2014; Ventripila marginata Roewer, 1917; Hutamaia plei Tourinho \& Mendes, 2014; Obidosus boibumba (Villarreal \& Pintoda-Rocha, 2006) y Stenostygnus pusio Simon, 1879.

Se encontraron ejemplares de Paecilaema bilunatum (Wood, 1869) (Cosmetidae) (Fig. 3i,) que tiene un registro incierto para Perú o Ecuador (Kury 2003a), aquí se confirma su presencia en Perú con dos machos adultos provenientes de la localidad de Aguas Negras (provincia de Maynas) (Tabla 3, Anexo 1). Asimismo, la ocurrencia de Paecilaemella multimaculata (Wood, 1869) (Cosme- 
Tabla 3. Registros de opiliones Laniatores en el departamento de Loreto examinados de la colección MUSM. AA: Alto Amazonas, R: Requena, U: Ucayali. Los números representan la cantidad de individuos registrados de cada especie por localidad. *Nuevos registros para Perú.

\begin{tabular}{|c|c|c|c|c|c|c|c|c|c|c|c|c|c|c|c|}
\hline & \multirow{2}{*}{ 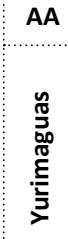 } & \multicolumn{5}{|c|}{ Loreto } & \multicolumn{6}{|c|}{ Maynas } & \multirow{2}{*}{ 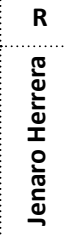 } & \multirow{2}{*}{ 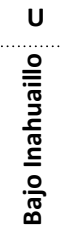 } & \multirow[b]{2}{*}{$\begin{array}{l}\bar{\pi} \\
\stackrel{0}{0}\end{array}$} \\
\hline & & 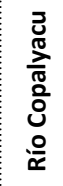 & $\begin{array}{l}\frac{0}{10} \\
\frac{0}{2} \\
\frac{0}{x}\end{array}$ & 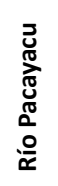 & 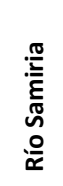 & 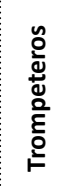 & 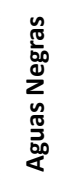 & $\begin{array}{l}\stackrel{n}{0} \\
\stackrel{+}{\overrightarrow{3}} \\
\underline{\sigma}\end{array}$ & $\begin{array}{l}\frac{\pi}{0} \\
\frac{\pi}{3} \\
\frac{0}{\alpha} \\
\frac{0}{\alpha}\end{array}$ & $\frac{\stackrel{0}{\pi}}{\frac{\pi}{0}}$ & $\begin{array}{l}\frac{0}{\pi} \\
\frac{1}{2} \\
\frac{0}{x}\end{array}$ & 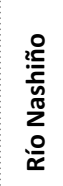 & & & \\
\hline \multicolumn{16}{|l|}{ GONYLEPTOIDEA } \\
\hline Gonyleptoidea 2 & & & & & & & & 3 & & & & & & & 3 \\
\hline Gonyleptoidea 4 & & & & & & & & & 1 & & & & & & 1 \\
\hline AGORISTENIDAE & & & & & 3 & & & & & & 2 & 1 & 1 & & 7 \\
\hline Avima 1 & & & & & 3 & & & & & & & & 1 & & 4 \\
\hline Avima 2 & & & & & & & & & & & & 1 & & & 1 \\
\hline Avima 3 & & & & & & & & & & & 1 & & & & 1 \\
\hline Leiobuninae 1 & & & & & & & & & & & 1 & & & & 1 \\
\hline COSMETIDAE & & 5 & 1 & & 214 & 1 & 2 & & 22 & 14 & 39 & 4 & 78 & 1 & 381 \\
\hline aff. Cynortoplus albimaculatus Roewer, 1925 & & 3 & & & & & & & & & & & & & 3 \\
\hline Cocholla 1 & & & & & & & & & 3 & & & & & & 3 \\
\hline Cocholla 2 & & & & & & & & & & & & 1 & & & 1 \\
\hline *Cocholla simoni Roewer, 1928 & & 2 & & & 18 & & & & & & 3 & & 1 & 1 & 25 \\
\hline Cosmetidae 2 & & & & & 3 & & & & & & & & 1 & & 4 \\
\hline Cosmetidae 9 & & & & & & & & & 11 & & 11 & & & & 22 \\
\hline Cosmetidae 10 & & & & & 83 & & & & & & 3 & & & & 86 \\
\hline Cosmetidae aff. 10 & & & & & & 1 & & & & & & & & & 1 \\
\hline Cosmetidae 11 & & & & & & & & & 1 & & 3 & & & & 4 \\
\hline Cosmetidae 13 & & & & & & & & & 1 & & 1 & & & & 2 \\
\hline Cosmetidae 14 & & & & & 7 & & & & & & & & & & 7 \\
\hline Cosmetidae 15 & & & & & & & & & & & 1 & & & & 1 \\
\hline Cosmetidae 21 & & & & & 9 & & & & & & & & & & 9 \\
\hline Cosmetidae 24 & & & 1 & & & & & & & & & & & & 1 \\
\hline *Cynorta marginalis Banks, 1909 & & & & & & & & & & 3 & & & 10 & & 13 \\
\hline *Discosomaticus cinctus (Perty, 1833) & & & & & & & & & & & & & 17 & & 17 \\
\hline Eulibitia aff. pollux Medrano \& Kury, 2017 & & & & & 32 & & & & & & & & & & 32 \\
\hline Meterginus cf. serratus Roewer, 1912 & & & & & 1 & & & & & & & & & & 1 \\
\hline *Meterginus serratus Roewer, 1912 & & & & & & & & & & 9 & 5 & 2 & 3 & & 19 \\
\hline Paecilaema bilunatum (Wood, 1869) & & & & & & & 2 & & & & & & & & 2 \\
\hline Paecilaemella multimaculata (Wood, 1869) & & & & & & & & & 1 & 2 & 2 & & & & 5 \\
\hline *Paecilaemula argentinoi Soares, 1970 & & & & & & & & & & & & & 43 & & 43 \\
\hline *Sibambea rotunda Roewer, 1917 & & & & & & & & & 1 & & 1 & 1 & & & 3 \\
\hline Taito aff. insperatus Kury \& Barros, 2014 & & & & & & & & & 1 & & 4 & & & & 5 \\
\hline *Taito insperatus Kury \& Barros, 2014 & & & & & & & & & 3 & & 5 & & & & 8 \\
\hline *Taito rorschachi Kury \& Barros, 2014 & & & & & 48 & & & & & & & & 3 & & 51 \\
\hline Taito sp.n. & & & & & 13 & & & & & & & & & & 13 \\
\hline \multicolumn{16}{|l|}{ CRANAIDAE } \\
\hline Cranaidae gen.n. & & & & & & & & & 1 & & & & & & 1 \\
\hline Phareicranaus 1 & & & & & & & & & & 3 & & & & & 3 \\
\hline Phareicranaus 2 & 1 & & & & & & & & 3 & & 1 & & & & 5 \\
\hline Phareicranaus 3 & & & & & & & & & 1 & & & & & & 1 \\
\hline Phareicranaus aff. calcarifer (Simon, 1879) & & & & & & & & & & 7 & & & & & 7 \\
\hline Phareicranaus cf. manauara (Pinto-da-Rocha, 1994) & & & & & & & & & & 1 & & & & & 1 \\
\hline Phareicranaus singularis (H. Soares, 1970) & & & & & & & & & & & 2 & & & & 2 \\
\hline *Ventripila marginata Roewer, 1917 & & & & & 1 & & & & 2 & & 7 & & & & 10 \\
\hline \multicolumn{16}{|l|}{ GONYLEPTIDAE } \\
\hline Gonyleptidae 1 & & & & & & & & & & & 3 & 2 & & & 5 \\
\hline Gonyleptidae 2 & & & & & & & & & 1 & & & & & & 1 \\
\hline *Hutamaia plei Tourinho \& Mendes, 2014 & & & & & 2 & & 1 & & & & 1 & 6 & 1 & & 11 \\
\hline Thaumatocranaus sp.n. & & & & & & & & & 3 & & 3 & & & & 6 \\
\hline \multicolumn{16}{|l|}{ MANAOSBIIDAE } \\
\hline Manaosbiidae 1 & & & & & 3 & & & & 3 & & 10 & & & & 16 \\
\hline NOMOCLASTIDAE & & & & & & & & & & & & & & & \\
\hline
\end{tabular}




\begin{tabular}{|c|c|c|c|c|c|c|c|c|c|c|c|c|c|c|c|}
\hline & \multirow{2}{*}{ 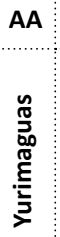 } & \multicolumn{5}{|c|}{ Loreto } & \multicolumn{6}{|c|}{ Maynas } & \multirow{2}{*}{ 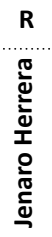 } & \multirow{2}{*}{$\begin{array}{c}\text { U } \\
\text { 음 } \\
\overline{\bar{N}} \\
\frac{D}{\pi} \\
\frac{\Xi}{0} \\
\stackrel{0}{\pi}\end{array}$} & \multirow[b]{2}{*}{ 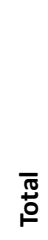 } \\
\hline & & 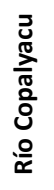 & $\begin{array}{l}\stackrel{\circ}{\circ} \\
\frac{\circ}{\pi} \\
\frac{0}{x}\end{array}$ & 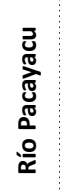 & 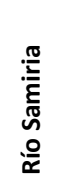 & 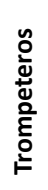 & 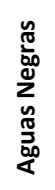 & 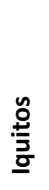 & 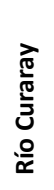 & 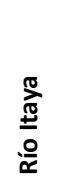 & $\begin{array}{l}\circ \\
\frac{0}{\pi 0} \\
\frac{0}{2} \\
\frac{0}{x}\end{array}$ & 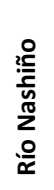 & & & \\
\hline Callcosma cofan Pinto-da-Rocha \& Bragagnolo, 2017 & & & & & 50 & & & & & & & & & & 50 \\
\hline \multicolumn{16}{|l|}{ STYGNIDAE } \\
\hline Eutimesius simoni Roewer, 1913 & & & & & & 1 & & & 2 & & 2 & & 7 & & 12 \\
\hline Obidosus 1 & & & & & & & & & 1 & & & & & & 1 \\
\hline Obidosus albilineatus Roewer, 1957 & & & & & & & & & & 5 & 5 & & & & 10 \\
\hline *Obidosus boibumba (Villarreal \& Pinto-da-Rocha, 2006) & 1 & & & & & & & & & & & & & & 1 \\
\hline Stygnus 1 & & & & & 2 & & & & & & 2 & 1 & & & 5 \\
\hline Stygnus klugi (Goodnight \& Goodnight, 1943) & 1 & & & 1 & 4 & & & & 1 & 1 & 4 & & 4 & 1 & 17 \\
\hline \multicolumn{16}{|l|}{ SAMOOIDEA } \\
\hline Samooidea 1 & & & & & & & & & & & 1 & & & & 1 \\
\hline \multicolumn{16}{|l|}{ BIANTIDAE } \\
\hline *Stenostygnus pusio Simon, 1879 & & & & & 1 & & & 1 & & & & & & & 2 \\
\hline \multicolumn{16}{|l|}{ SAMOIDAE } \\
\hline Samoidae 1 & & & & & 33 & & & & & & & & & & 33 \\
\hline \multicolumn{16}{|l|}{ STYNOMMATIDAE } \\
\hline Stygnomma 1 & & & & & 2 & & 2 & & & & & & & & 4 \\
\hline \multicolumn{16}{|l|}{ ZALMOXOIDEA } \\
\hline \multicolumn{16}{|l|}{ ZALMOXIDAE } \\
\hline Zalmoxidae 1 & & & & & 127 & & & & & & & & & & 127 \\
\hline Zalmoxidae 2 & & & & & 2 & & & & & & & & & & 2 \\
\hline Zalmoxidae 3 & & & & & & & & & 1 & & & & & & 1 \\
\hline Zalmoxidae 4 & & & & & 21 & & & & & & & & & & 21 \\
\hline Total & 3 & 5 & 1 & 1 & 465 & 2 & 5 & 4 & 42 & 31 & 82 & 14 & 91 & 2 & 748 \\
\hline
\end{tabular}

tidae) (Fig. 3g), reportada como incierta en Perú "Napo and Maravon [Napo and Marañon rivers]" (Medrano et al. 2020), es confirmada por la presencia de dos ejemplares provenientes del río Napo, uno de río Curaray y otros dos del río Itaya (Tabla 3, Anexo 1).

La familia más diversa (Tablas 2 y 3 ) es Cosmetidae (34 spp.), comprendiendo el $47 \%$ de todos los registros examinados, seguida por Cranaidae (11 spp.) y Stygnidae (8 spp.). La familia Cosmetidae también destacó por la gran variedad de formas que exhibe (Fig. 3), en algunas especies como resultado de un dimorfismo sexual muy marcado (Figs. 4c, d).

En relación con el río Samiria (Tabla 3, Anexo 1), de un total de 465 individuos examinados en la colección MUSM, más Phareicranaus hermosa (Pinto-da-Rocha \& Kury, 2003) reportada en base a esas muestras, se encontraron 23 especies de Laniatores distribuidas en 11 familias. En cuanto a la riqueza, destaca también la familia Cosmetidae con nueve especies (aprox. 41\%), seguida por Zalmoxidae con tres especies.

En términos de abundancia para el material examinado, nuevamente figura Cosmetidae con 214 individuos (aprox. 46\%), seguida por Zalmoxidae con 150 (aprox. $32 \%)$. Los Laniatores del río Samiria parecen exhibir un alto grado de endemismo local, de las especies encontradas, 10 de ellas (aprox. 43\%) ocurren solo en esta localidad; sin embargo, hasta el momento, con certeza, solo se puede afirmar que por lo menos una de ellas, Taito sp. n. (Cosmetidae) es nueva para ciencia.

Notas de distribución por provincias. - Dentro del departamento de Loreto, el mayor número de registros de Laniatores (Tabla 1, Fig. 5) se tiene en la provincia de Maynas (46 spp.) y en la provincia de Loreto (27 spp.). No hay registros de Laniatores para la provincia de Putumayo, y solo se tienen dos registros tanto para el Dátem del Marañón como para la provincia de Ucayali (Tabla 1, Fig. 5).

Con relación a las localidades (Tablas 1 y 3), tres de ellas exhibieron una mayor riqueza de Laniatores: río Napo (26 spp.), río Samiria (23 spp.) y río Curaray (20 spp.).

En casi todas las provincias, la familia Cosmetidae está ampliamente distribuida, aquí se registra en 12 de las 18 localidades estudiadas (Tabla 1, Tabla 3).

Algunas especies tienen registros en tres o más provincias (Tabla 3), por ejemplo, Eutimesius simoni, Stygnus klugi, Meterginus serratus y Cocholla simoni (v. g. Fig. 6). En contraste, otras especies se restringen a una sola localidad, como es el caso de ocho especies registradas solo para Pebas, entre ellas, Pebasia singularis, Tegyra cinnamomea y Poecilaemula peruviana; y 10 especies que ocurren solo en Cocha Shinguito, río Samiria (v.g. Fig. 7), como es el caso de Cosmetidae 14, Samoidae 1 y Zalmoxidae 1, entre otras. 


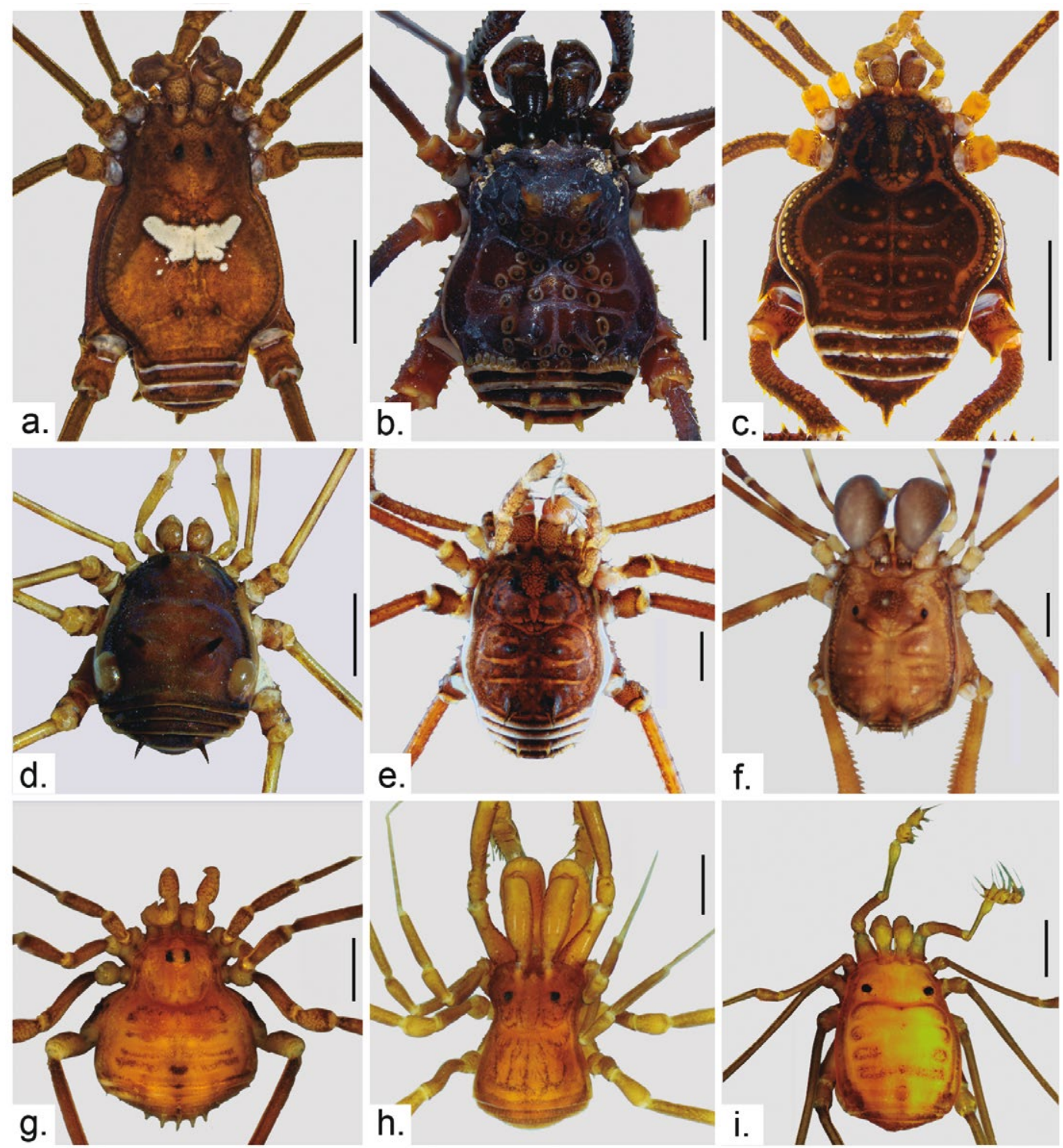

Figura 2. Familias de opiliones Laniatores presentes en Loreto. (a) Cosmetidae, Taito rorschachi, $q$ MUSM-ENT 0514108 (b) Cranaidae, Phareicranaus singularis, ô MUSM-ENT 0511981. (c) Gonyleptidae, Hutamaia plei, ô MUSM-ENT 0511946. (d) Nomoclastidae, Callcosma cofan, + MUSM-ENT 0512000. (e) Manaosbiidae 1, q MUSM-ENT 0512090 (f) Stygnidae, Stygnus klugi, ơ MUSM-ENT 0507500. (g) Zalmoxidae 1, ô MUSM-ENT 0514511. (h) Stygnommatidae, Stygnomma 1, đ̂ MUSM-ENT 0514513. (i) Biantidae, Stenostygnus pusio, o MUSM-ENT 0511812. Barras de escala: a-c $=3 \mathrm{~mm}, \mathrm{~d}-\mathrm{h}=1 \mathrm{~mm}, \mathrm{i}=0.5 \mathrm{~mm}$.

\section{Discusión}

Este trabajo registra en el departamento de Loreto 72 especies distribuidas en 11 familias del suborden Laniatores, de las cuales solo 19 han sido reportadas previamente en la literatura (Kury 2003a, Pinto-da-Rocha \& Kury 2003, Hara et al. 2014, Ochoa \& Pinto-da-Rocha 2014, Pinto-da-Rocha \& Bragagnolo 2017, Pinto-da-Rocha \& Bonaldo 2011, Villarreal et al. 2019; Medrano et al. 2020, Pinzón-M et al. 2021). Estos números reflejan una baja diversidad, pero queda claro que los pocos registros que se tienen son resultado de los escasos muestreos enfocados a la búsqueda de arácnidos en Loreto. Un claro ejemplo del efecto del muestreo que refleja la riqueza de la opiliofauna se tiene en el río Samiria con 23 especies distribuidas en 11 familias de Laniatores; estos resultados son en parte comparables con aquellos enfocados en Laniatores en la Reserva Natural Río Nambí en
Colombia (12 familias y aprox. 28 especies; García \& Medrano 2015) y algunos citados para la Amazonía brasileña como, por ejemplo, río Jurití en el estado de Pará (25 especies; Pinto-da-Rocha \& Bonaldo 2006) y el Parque da Onça Parda en São Paulo (25 especies; Resende et al. 2012). No obstante, hay otras localidades brasileñas mucho más ricas en opiliones, como el Parque Nacional Serra dos Orgãos en la Mata Atlántica, Río de Janeiro, donde se han registrado 54 especies de Laniatores (Bragagnolo \& Pinto-da-Rocha 2003). En general, estos altos valores por localidad son resultado de muestreos intensivos diseñados por aracnólogos para conocer la opiliofauna de dichas localidades, pero más importante, interesados en esclarecer las relaciones evolutivas de algunos de los linajes que allí ocurren.

Aunque las muestras no son comparables dado que las técnicas y el esfuerzo de colecta no son similares, los 

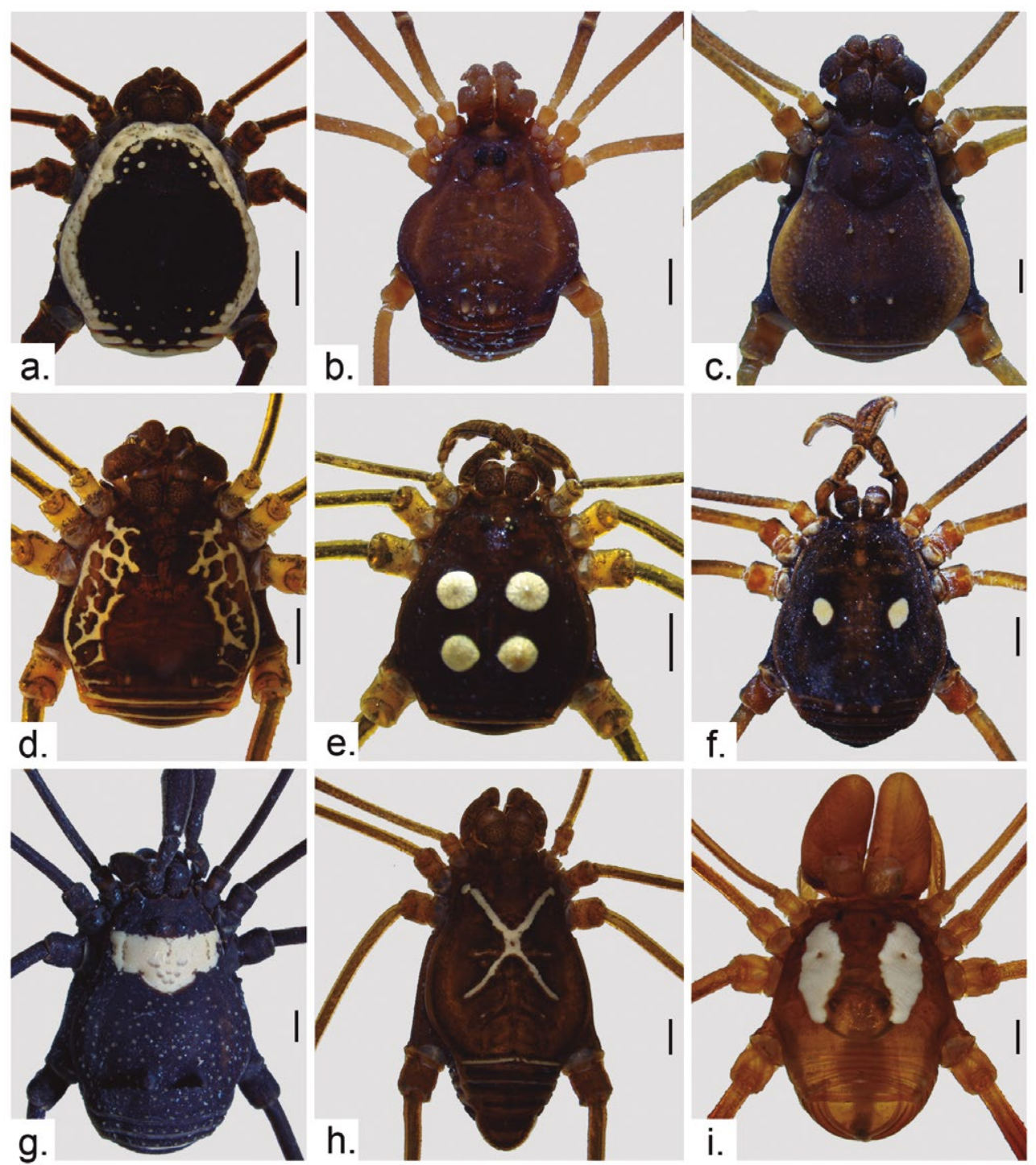

Figura 3. Diversidad de formas y patrón de ornamentación en Cosmetidae. (a) Sibambea rotunda, of MUSM-ENT 0514096. (b) Eulibitia cf. pollux, \& MUSM-ENT 0512586. (c) Cosmetidae 10, ô MUSM-ENT 0514510. (d) Cosmetidae 13, + MUSM-ENT 0512184. (e) Cosmetidae 15, ô MUSM-ENT 0514087. (f) Cocholla simoni, ô MUSM-ENT 0512575. (g) Paecilaemella multiloculata, o MUSM-ENT 0514512. (h) Cosmetidae 2, ô MUSMENT 0512431. (i) Paecilaema bilunatum, ơ MUSM-ENT 0512589. Barras de escala = $1 \mathrm{~mm}$.

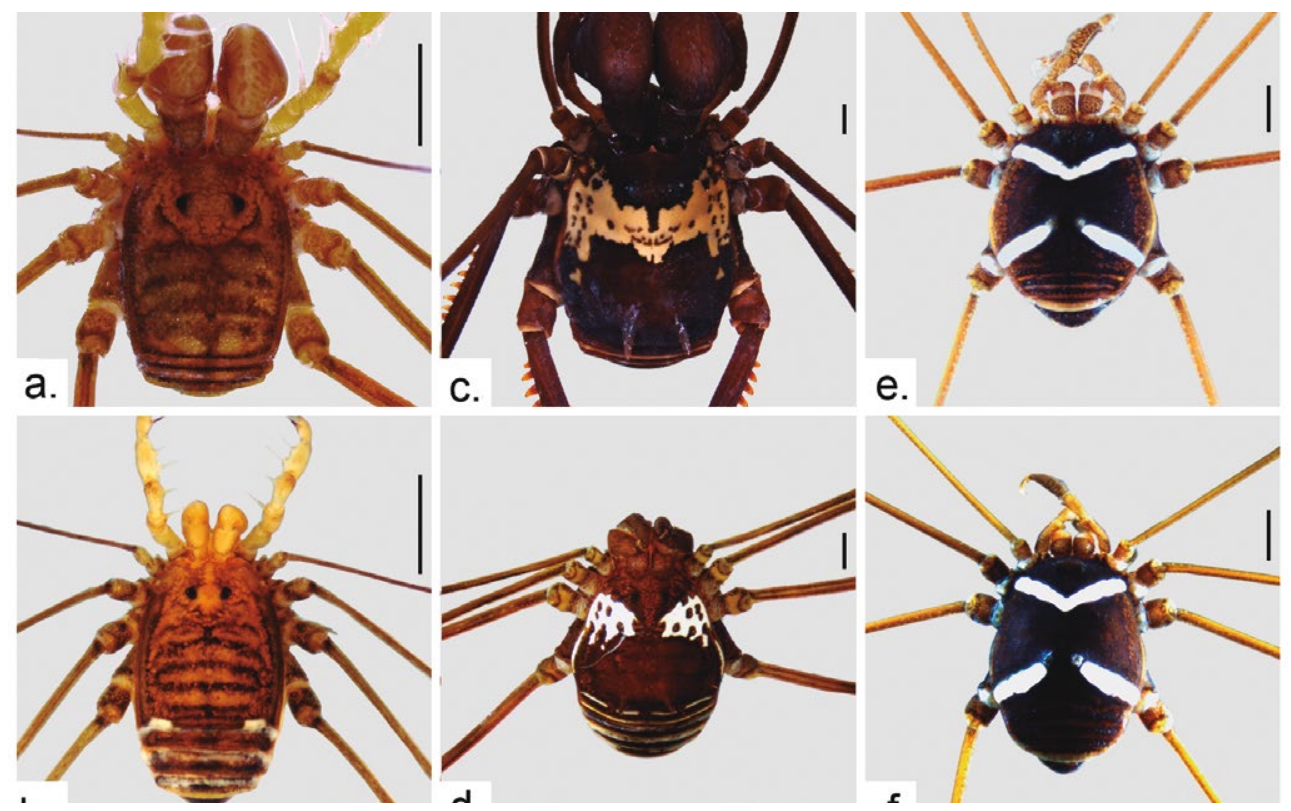

Figura 4. Dimorfismo sexual. (a y b) Avima 1, (a) macho con quelíceros engrosados, MUSM-ENT 0511900. (b) hembra, con quelíceros simples, MUSM-ENT 0511898. (c y d) Meterginus serratus, (c) macho con quelíceros hipertélicos y fémures III y IV con espinas, MUSM-ENT 0512593; (d) hembra con quelíceros y fémures normales, MUSM-ENT 0512449. (e y f) Paecilaema argentinoi, sin dimorfismo, (e) macho, MUSM-ENT 0514172; (f) hembra, MUSM-ENT 0512590. Barras de escala $=1 \mathrm{~mm}$. 


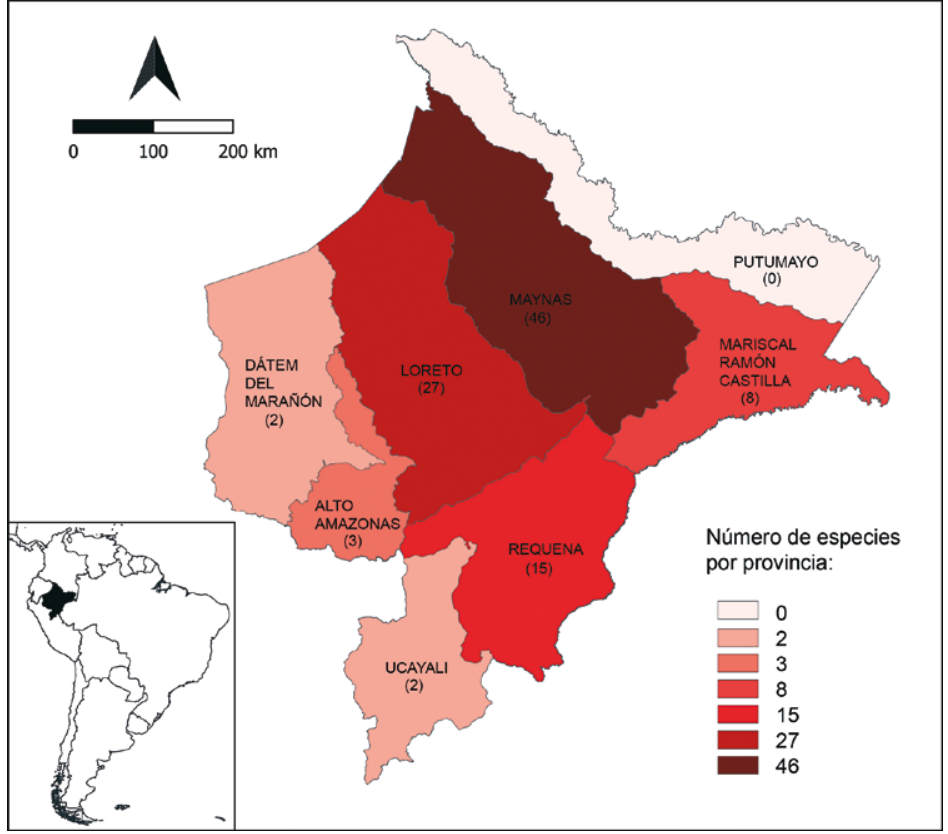

Figura 5. Registros de opiliones Laniatores en las provincias de Loreto.

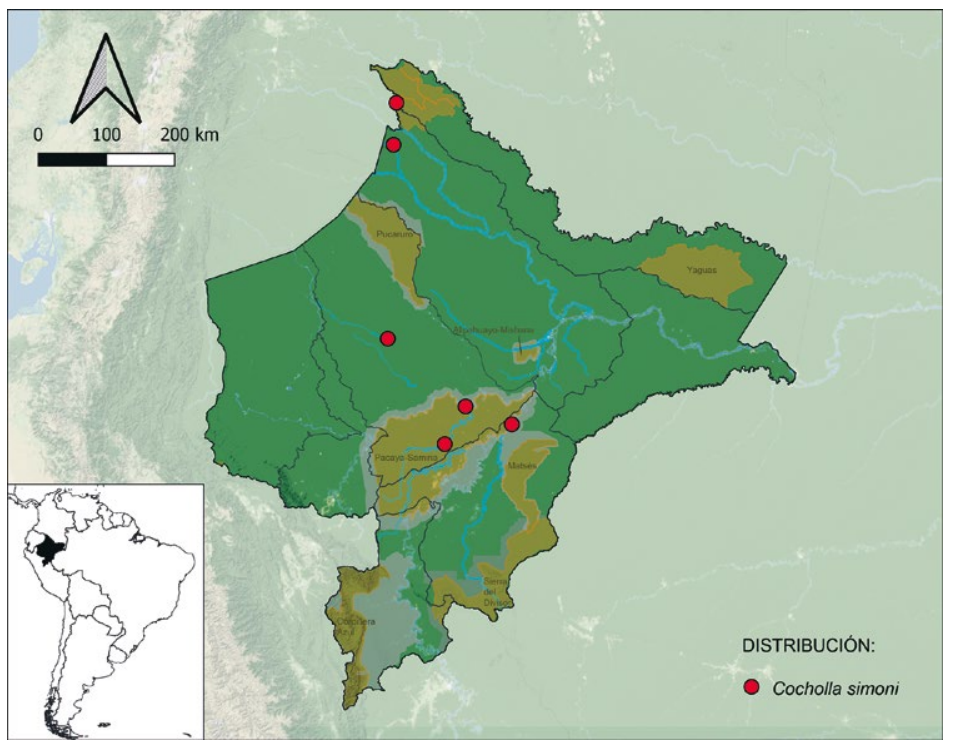

Figura 6. Registros de Cocholla simoni (Cosmetidae) en Loreto.

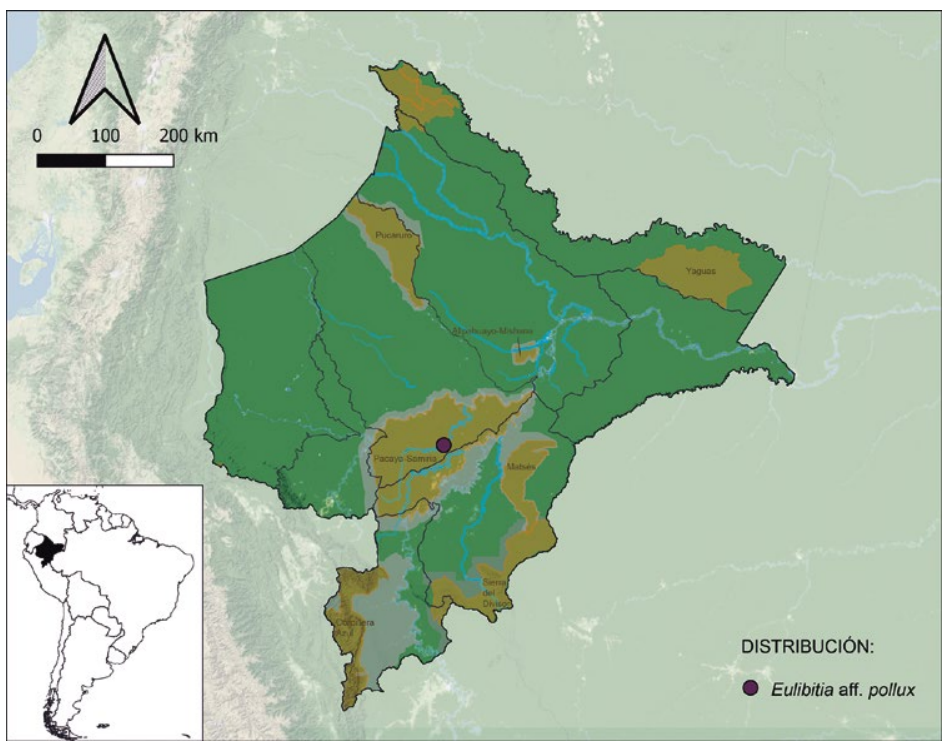

Figura 7. Registros de Eulibitia aff. pollux (Cosmetidae) en Loreto. 
datos de riqueza y abundancia de Laniatores obtenidos en el río Samiria permiten tener una idea de la composición de la comunidad en estudio, aunque se requiere más información para validar las conclusiones. Así se tiene que en Samiria hay 11 familias de Laniatores, siendo la más diversa Cosmetidae ( 9 spp.), concordando con el patrón observado de una mayor riqueza hacia el norte de Sudamérica (Giribet \& Kury 2007); los resultados en Jurití (Pinto-da-Rocha \& Bonaldo 2006) muestran ocho familias registradas y las más ricas son Cosmetidae ( 7 spp.) y Zalmoxidae (6 spp.) por lo que concuerdan en parte con lo encontrado en el río Samiria, donde Zalmoxidae está representada por tres especies.

Aún falta un mayor estudio, principalmente enfocado en revisiones taxonómicas, para determinar a nivel de especie las 43 morfoespecies asignadas, algunas de las cuales podrían ser nuevas para la ciencia (Anexo 1), como en el caso de los cosmétidos no identificados (Medrano, com. personal) o constituir nuevos registros para el país.

Los registros de Laniatores en la mayoría de las 18 localidades estudiadas han sido producto de colectas accidentales u oportunistas; sin embargo, en cuatro de ellas (río Samiria, río Napo, río Curaray y Jenaro Herrera) sí hubo un muestreo dirigido a la búsqueda de arácnidos, aunque los objetivos del diseño muestral fueron diferentes. Solo en río Samiria y Jenaro Herrera se trazó como meta inventariar la aracnofauna de dichas localidades, pero con un menor esfuerzo de colecta en el último. Asimismo, llama la atención que entre las especies dominantes en el río Samiria (aquellas con más de 50 individuos, Tabla 3) se encuentra una especie de Zalmoxidae, familia que por lo general se conoce de lotes muy pequeños (Pérez-Gonzáles, com. pers.); si bien es posible que se deba a la metodología utilizada, nebulización del dosel arbóreo, no hay referencia al uso de esta técnica en otros estudios sobre opiliones; más aún, los zalmoxoideos en general son reportados en hábitats de hojarasca (Acosta et al. 2007) por lo que su ocurrencia en doseles arbóreos podría tratarse de una novedad a tenerse en cuenta para la ecología de este grupo.

Aún falta mucho por estudiar para poder establecer los patrones de distribución de las especies de Laniatores que ocurren en Loreto; sin embargo, aquí se registran algunas especies con distribuciones aparentemente amplias pero que podrían corresponder a patrones particulares, por ejemplo, Obidosus albilineatus Roewer 1957, está registrada para Brasil, Ecuador y Perú, reportándose en este último en Ucayali y Madre de Dios (Villarreal et al. 2019); Eutimesius simoni Roewer, 1913, ocurre en Brazil, Colombia, Ecuador y Perú (Pinto-daRocha 1997), en lo que parece ser la cuenca del Napo; en el caso de Iquitosa poecilis Roewer, 1943 (Hara et al. 2014) los registros corresponden a Loreto, Cusco, Pasco y Huánuco, todos en bosques bajos de los Andes orientales de Perú; finalmente, aunque endémico de Perú, Stygnus klugi (Goodnight \& Goodnight 1943) está registrada para Amazonas, Huánuco y ampliamente distribuido en Loreto (Kury 2003a).
La información reunida hasta la fecha muestra que hay 42 especies de Laniatores que ocurren solo en el departamento de Loreto, algunas de ellas incluso exhiben distribuciones mucho más localizadas, como las 10 especies que se restringen solo a los bosques del Río Samiria, o las siete conocidas solo para Pebas. Es interesante contrastar estos resultados con lo hallado por Ruokolainen et al. (2002) para algunos grupos de plantas, entre ellas, Melastomatacea y Pteridophyta; ellos indican que el error en interpretar los patrones de distribución puede deberse 1) a que el foco se centra en especies de gran tamaño, donde si es posible ver un mayor número de especies con distribuciones muy amplias y 2) al número de especies identificadas vs. el número de morfoespecies, estas últimas tienden a exhibir distribuciones mucho más restringidas, por ejemplo, al comparar grupos como Melastomatacea (arbustos y herbáceas) y Pteridophyta (helechos) en tres regiones de Sudamérica, ellos encontraron que las especies identificadas tenían mayormente distribuciones más amplias, así, el 59\% de las melastomatáceas y el 69\% de pteridofitas ocurrían en Loreto, Madre de Dios y en Ecuador (Yasuní) mientras que el $84 \%$ de las morfoespecies de Melastomatacea y el 75\% de pteridofitas se registraron en una sola región. Finalmente, los autores afirman que estos dos sesgos en interpretación de rangos de distribución afectarían también a las comunidades de fauna y deben tomarse en cuenta para estudios de comunidades tropicales y una interpretación más precisa de sus patrones de distribución.

En conclusión, todavía hay vacíos muy grandes en el departamento de Loreto, en general, se requiere de esfuerzos de muestreo más exhaustivos y estandarizados para reunir información con datos comparables; igualmente, las técnicas de colecta deben dirigirse puntualmente a microhábitats que permitan descubrir/ monitorear diferentes grupos taxonómicos a través del tiempo y espacio de tal modo que se pueda conocer la dinámica de sus poblaciones y examinar en más detalle sus variaciones morfológicas, y finalmente establecer con mayor precisión su identidad taxonómica y rango de distribución.

\section{Literatura citada}

Acosta L, Machado G. 2007. Diet and Foraging. In: R. Pinto-daRocha, G Machado and G. Giribet, eds. Harvestmen: the biology of Opiliones. Harvard University Press. Pp. 309-338.

Acosta L, Pérez-González A, Tourinho A. 2007. Methods for taxonomic study. In: R. Pinto-da-Rocha, G Machado and G. Giribet, eds. Harvestmen: the biology of Opiliones. Harvard University Press. Pp. 494-505.

Adis J. 2002. Taxonomical classification and biodiversity. In: J. Adis ed. Amazonian Arachnida and Myriapoda. Pensoft Publisher. Pp: 13-15.

Bragagnolo C, Pinto-da-Rocha R. 2003. Diversidade de opiliões do parque nacional da Serra dos Orgãos, Río de Janeiro, Brasil (Arachnida: Opiliones). Biota Neotropica. 3(1): 1-20. https://doi.org/10.1590/S1676$\underline{06032003000100009 .}$. 
Chamberlin R. 1916. Results of the Yale Peruvian expedition of 1911. The Arachnida. Bulletin of the Museum of Comparative Zoology at Harvard University, 60(6): 175199. Pls. $1-25$

Curtis D, Machado G. 2007. Ecology. In: R. Pinto-da-Rocha, G Machado and G. Giribet, eds. Harvestmen: the biology of Opiliones. Harvard University Press. Pp. 280-308.

DaSilva M, Pinto-da-Rocha R, DeSouza A. 2015. A protocol for the delimitation of areas of endemism and the historical regionalization of the Brazilian Atlantic Rain Forest using harvestmen distribution data. Cladistics 31: 692-705. https://doi.org/10.1111/cla.12121.

García A, Medrano M. 2015. Orden Opiliones. In: E. Flórez, C Romero, DS López, eds. Los Artrópodos de la reserva Natural Río Ñambí. Serie de Guías de Campo del Instituto de Ciencias Naturales No. 15. Universidad Nacional de Colombia, Bogotá. Pp. 109-126.

Giribet G, Kury A. 2007. Phylogeny and Biogeography. In: R. Pinto-da-Rocha, G Machado and G. Giribet, eds. Harvestmen: the biology of Opiliones. Harvard University Press. Pp. 208-308.

Guizado J, Girard D. 1966. Reconocimiento por calizas en la región de Orellana - Cushabatay (Prov. de Ucayali, departamento de Loreto). INGEMMET. Boletín, Serie A: Carta Geológica Nacional; no 13: 259-272. https://hdl. handle.net/20.500.12544/129.

Hara M, Pinto-da-Rocha R, Villarreal O. 2014. Revision of the cranaid genera Phalangodus, Iquitos and Aguaytiella (Opiliones: Laniatores: Gonyleptoidea). Zootaxa 3814(4): 537-580. https://doi.org/10.11646/zootaxa.3814.4.8.

Kury A. 2003a. Annotated catalogue of the Laniatores of the New World (Arachnida, Opiliones). Revista Ibérica de Aracnología 7: 1-325.

Kury A. 2003b. OmniPaper Project-The ARACNOLAB internet resource on Opilionological Taxonomic Literature. $\mathrm{Mu}$ seu Nacional/UFRJ website. Online at: <http://www. museunacional.ufri.br/mndi/Aracnologia/pdfliteratura/pdfs\%20opiliones.htm>. Acceso 15/12/2020.

Kury A. 2013. Order Opiliones Sundevall, 1833. In: Zhang, Z.Q. (Ed.) Animal Biodiversity: An Outline of Higherlevel Classification and Survey of Taxonomic Richness (Addenda 2013). Zootaxa, 3703: 1-82. https://doi. org/10.11646/zootaxa.3703.1.7.

Kury A. 2020. (en línea) Checklist of valid genera of Opiliones of the World. Museu Nacional/UFRJ website. Online at: $<$ http://www.museunacional.ufrj.br/mndi/Aracnologia/checklaniator.htm $>$ _.Acceso 15/12/2020.

Kury A, Alonso-Zarazaga A. 2011. Addenda and corrigenda to the "Annotated catalogue of the Laniatores of the New World (Arachnida, Opiliones)". Zootaxa 3034: 47-68. https://doi.org/10.11646/zootaxa.3034.1.3.

Kury A, Barros C. 2014. A new genus and eight new species of Amazonian cosmetines (Opiliones, Laniatores, Cosmetidae). Zoological Studies 2014, 53:24. https://doi. org/10.1186/s40555-014-0024-4.

Kury A, Mendes A, Cardoso L, Kury M, Granado A. 2020. WCOLite: online world catalogue of harvestmen (Arachnida, Opiliones). Version 1.0 - Checklist of all valid nomina in Opiliones with authors and dates of publication up to 2018. Self published, Río de Janeiro, ii+237pp. https://doi.org/10.5281/zenodo.4025288.

Kury A, Pinto-da-Rocha R. 2007. Cosmetidae Koch, 1839. In: R. Pinto-da-Rocha, G Machado and G. Giribet, eds. Harvestmen: the biology of Opiliones. Harvard University
Press. Pp. 182-185.

Medrano M, De Ázara L, Kury A. 2020. The short-legged Andean cosmetids revisited: the genus Libita Simon, 1879 with description of two new species (Opiliones, Cosmetidae). European Journal of Taxonomy 634: 1-25. https://doi.org/10.5852/ejt.2020.634.

Mello-Leitão CF. 1949. Famílias, Subfamília, Espécies e Géneros novos de Opilioes e notas de sinonimia. Boletim do Museu Nacional do Río de Janeiro 94: 1-33.

Ochoa JA, Pinto-da-Rocha R. 2014. Three new Peruvian species of Protimesius (Opiliones: Laniatores; Stygnidae). Journal of Arachnology 41(2): 197-204.

Pinto-da-Rocha R. 1997. Systematic review of the neotropical family Stygnidae (Opiliones, Laniatores, Gonyleptoidea). Arquivos de Zoologia. 33(4):163-342.

Pinto-da-Rocha R, Bonaldo A. 2006. A structured inventory of harvestmen (Arachnida, Opiliones) at Juruti River plateau, State of Pará, Brazil. Revista Ibérica de Aracnología 13: 155-162. http://repositorío.museu-goeldi.br/ handle/mgoeldi/305.

Pinto-da-Rocha R, Bonaldo A. 2011. Species relationships in the Neotropical genus Phareicranaus Roewer 1913 (Opiliones: Cranaidae): two new species and new data from Penial morphology. Zootaxa 3134:1-34. https:// doi.org/10.11646/zootaxa.3135.1.1.

Pinto-da-Rocha R, Bragagnolo C. 2017. Cladistic analysis of the family Nomoclastidae with descriptions of a new genus and eight new species (Opiliones, Laniatores). Invertebrate Systematics 31(1): 91-123. https://doi. org/10.1071/IS15050.

Pinto-da-Rocha R, Kury A. 2003. Phylogenetic analysis of Santinezia with description of five new species (Opiliones, Laniatores, Cranaidae). The Journal of Arachnology 31:173-208. https://doi.org/10.1636/0161-8202(20 03)031[0173:PAOSWD]2.0.C0;2.

Pinzón-M C, Damron B, Pinto-da-Rocha R. 2021. Three new species of Cosmetidae C.L. Koch, 1839 from South America (Opiliones: Cosmetidae). Zootaxa 4989 (1): 203217. https://doi.org/10.11646/zootaxa.4984.1.16.

Resende L, Pinto-da-Rocha R, Bragagnolo C. 2012. Diversity of harvestmen (Arachnida, Opiliones) in Parque da Onça Parda, southeastern Brazil. Iheringia, Série Zoologia 102(1): 99-105. https://doi.org/10.1590/S007347212012000100014

Roewer CF. 1929. Über Phalangodiden I. (Subfam. Phalangodinae, Tricommatinae, Samoinae.) Weitere Weberknechte XIII. Senckenbergiana biologica 30(1/3): 11-61.

Roewer CF. 1957. Arachnida Arthrogastra aus Peru, III. Senckenbergiana biologica 38(1/2): 67-94.

Ruokolainen K, Tuomisto H, Vormisto J, Pitman N. 2002. Two biases in estimating range sizes of Amazonian plant species. Journal of Tropical Ecology 18(6): 935-942. https://doi.org/10.1017/S0266467402002614.

Silva D. 1996. Species composition and community structure of Peruvian rainforest spiders: a case study from a seasonally inundated forest along the Samiria river. Revue suisse de Zoologie 2: 597-610.

Soares H, Bauab-Vianna M. 1973. Contribuição ao estudo dos Opiliões do Peru (Opiliones: Gonyleptidae). Acta Zoologica Lilloana 29: 317-342.

Southwood TRE, Moran VC, Kennedy CEJ. 1982. The richness, abundance and biomass of the arthropod communities on trees. Journal of Animal Ecology 51: 635-649. https://doi.org/10.2307/3988. 
Villarreal O, Ázara LN, Kury AB. 2019. Revalidation of Obidosus Roewer, 1913 and description of two new cave-dwelling species of Protimesius Roewer, 1913 from Brazil (Opiliones: Stygnidae). Journal of Natural History 53(15-16): 965-989. https://doi.org/10.1080/00222 933.2019.1620893.

Villarreal O, García A, Kury A. 2021. Fine tuning the diversity in four families of Gonyleptoidea (Arachnida, Opiliones) in Venezuela. Revista Mexicana de Biodiversidad 92: $1-22$.

\section{Agradecimientos / Acknowledgments:}

AA-A agradece la colaboración de Miguel Medrano, Osvaldo Villarreal-Manzanilla, Marco Hara y Conchita Pinzón por su orientación en la identificación de material de este trabajo, también agradece a Abel Pérez-González por parte de aquellos "Zal-Sa" identificados parcialmente hasta el momento (Anexo 1). Las autoras agradecen a Gerardo Lamas por su apoyo en la ubicación de localidades y comentaríos en general; A Adriano Kury por el material bibliográfico proporcionado en OmniPaper Project. Se agradece a Wildlife Conservation Society y a Biósfera Consultores Ambientales S.A.C. por su contribución para el desarrollo del manuscrito. DS-D agradece también a Terry Erwin $\left({ }^{+}\right)$y a la Institución Smithsoniana, Washington DC, por todo el apoyo brindado para el trabajo de campo en el río Samiria. Quedamos en deuda con los revisores anónimos por sus valiosos comentaríos que mejoraron notablemente el producto de este trabajo.

\section{Conflicto de intereses / Competing interests:}

DS-D es editora de la RPB, no participó en el el proceso de edición. Los autores declaran que no incurren en conflictos de intereses.

\section{Rol de los autores / Authors Roles:}

DS-D y AA-A: conceptualización, análisis formal, escritura- preparación del borrador original, redacción: revisión y edición. AA-A: metodología, investigación y curación de datos. DS-D: recursos, administración de proyecto y adquisición de fondos.

\section{Fuentes de financiamiento / Funding:}

Las autoras declaran que este trabajo no recibió financiamiento específico de ninguna agencia, sector gubernamental o agencia sin fines de lucro.

Aspectos éticos / legales; Ethics / legals:

Las autoras declaran no haber incurrido en ningún aspecto antiético ni omitido norma legal nacional o internacional en la investigación y elaboración del artículo. 
Anexo 1. Material de opiliones Laniatores del departamento de Loreto examinados del MUSM. *Nuevos registros para Peru.

\begin{tabular}{|c|c|c|c|c|c|c|c|c|c|c|c|c|c|c|c|c|c|c|}
\hline \# & Código de ingreso & No. Ind & Especie/Morfoespecie & Provincia & Localidad & Lugar de colecta & $\stackrel{\text { Altitud }}{m}$ & Latitud & Longitud & Dia & Mes & Año & colectores & $\begin{array}{l}\text { Fuente para } \\
\text { georeferen- } \\
\text { ciación }\end{array}$ & Proyecto de colecta & Superfamilia & Familia & Identificación \\
\hline 1 & MUSM-ENT 0511891 & 1 & Avima 1 & Loreto & Río Samiria & Cocha Shinguito & 90 & $5^{\circ} 10^{\prime}$ & 74'39' & 27 & v & 1990 & T. Erwin & Etiqueta & $\begin{array}{l}\text { MHN-UNMSM/Smith- } \\
\text { sonian Inst. }\end{array}$ & Gonyleptoidea & Agoristenidae & A. Ayala-Apaza, 2021 \\
\hline 2 & MUSM-ENT 0511898 & 1 & Avima 1 & Loreto & Río Samiria & Pithecia & 111 & $5^{\circ} 10^{\prime}$ & 74'39' & 15 & viii : & 1989 & D. Silva & Etiqueta & $\begin{array}{l}\text { MHN-UNMSM/Smith- } \\
\text { sonian Inst. }\end{array}$ & Gonyleptoidea & Agoristenidae & A. Ayala-Apaza, 2021 \\
\hline 3 & MUSM-ENT 0511900 & 1 & Avima 1 & Requena & Jenaro Herrera & $\begin{array}{l}\text { Centro de investigación } \\
\text { Jenaror Herrera, en pal- } \\
\text { meras Astrocarium sp. }\end{array}$ & 100 & $04^{\circ} 55^{\prime}$ & $73^{\circ} 45^{\prime}$ & 23 & viii & 1988 & D. Silva & Etiqueta & MHN-UNMSM & Gonyleptoidea & Agoristenidae & A. Ayala-Apaza, 2021 \\
\hline 4 & MUSM-ENT 0511985 & 1 & Avima 1 & Loreto & Río Samiria & Cocha Shinguito & 90 & $5^{\circ} 10^{\prime}$ & $74^{\circ} 39^{\prime}$ & 22 & v & 1990 & D. Silva & Etiqueta & $\begin{array}{l}\text { MHN-UNMSM/Smith- } \\
\text { sonian Inst. }\end{array}$ & Gonyleptoidea & Agoristenidae & A. Ayala-Apaza, 2021 \\
\hline 5 & MUSM-ENT 0512121 & 1 & Avima 2 & Maynas & Rio Nashiño & & 190 & $1^{1023} 25.7^{\prime \prime}$ & $75^{\circ} 16^{\prime} 46.64 "$ & 25 & xii & 2008 & R. Yawarcani & Etiqueta & Consultoría EIA & Gonyleptoidea & Agoristenidae & A. Ayala-Apaza, 2021 \\
\hline 7 & MUSM-ENT 0512000 & 1 & Callcosma cofan Pinto-da-Rocha \& Bragagnolo, 2017 & Loreto & Río Samiria & Cocha Shinguito & 90 & $5^{\circ} 10^{\prime}$ & 74³9' & 30 & v & 1990 & D. Silva & Etiqueta & $\begin{array}{l}\text { MHN-UNMSM/Smith- } \\
\text { sonian Inst. }\end{array}$ & Gonyleptoidea & Nomoclastidae & $\begin{array}{l}\text { M. Medrano y A. Ayala-Apaza, } \\
2021\end{array}$ \\
\hline 8 & MUSM-ENT 0512004 & 10 & Callcosma cofan Pinto-da-Rocha \& Bragagnolo, 2017 & Loreto & Río Samiria & Cocha Shinguito & 90 & $5^{\circ} 10^{\prime}$ & 74³9' & 15 & v & 1990 & D. Silva & Etiqueta & $\begin{array}{l}\text { MHN-UNMSM/Smith- } \\
\text { sonian Inst. }\end{array}$ & Gonyleptoidea & Nomoclastidae & A. Ayala-Apaza, 2021 \\
\hline 9 & MUSM-ENT 0512492 & 10 & Callcosma cofan Pinto-da-Rocha \& Bragagnolo, 2017 & Loreto & Río Samiria & Cocha Shinguito & 90 & $5^{\circ} 10^{\prime}$ & 74'39' & & v & 1990 & T. Erwin & Etiqueta & $\begin{array}{l}\text { MHN-UNMSM/Smith- } \\
\text { sonian Inst. }\end{array}$ & Gonyleptoidea & Nomoclastidae & A. Ayala-Apaza, 2021 \\
\hline 10 & MUSM-ENT 0512526 & 22 & Callcosma cofan Pinto-da-Rocha \& Bragagnolo, 2017 & Loreto & Río Samiria & Cocha Shinguito & 90 & $5^{\circ} 10^{\prime}$ & 74³9' & 16 & v & 1990 & T. Erwin & Etiqueta & $\begin{array}{l}\text { MHN-UNMSM/Smith- } \\
\text { sonian Inst. }\end{array}$ & Gonyleptoidea & Nomoclastidae & A. Ayala-Apaza, 2021 \\
\hline 11 & MUSM-ENT 0512543 & 6 & Callcosma cofan Pinto-da-Rocha \& Bragagnolo, 2017 & Loreto & Río Samiria & Cocha Shinguito & 90 & $5^{\circ} 10^{\prime}$ & 74³9' & 15 & v & 1990 & D. Silva & Etiqueta & $\begin{array}{l}\text { MHN-UNMSM/Smith- } \\
\text { sonian Inst. }\end{array}$ & Gonyleptoidea & Nomoclastidae & A. Ayala-Apaza, 2021 \\
\hline 12 & MUSM-ENT 0514508 & 1 & Callcosma cofan Pinto-da-Rocha \& Bragagnolo, 2017 & Loreto & Río Samiria & Cocha Shinguito & 90 & $5^{\circ} 10^{\prime}$ & 74³9' & 30 & v & 1990 & D. Silva & Etiqueta & $\begin{array}{l}\text { MHN-UNMSM/Smith- } \\
\text { sonian Inst. }\end{array}$ & Gonyleptoidea & Nomoclastidae & A. Ayala-Apaza, 2021 \\
\hline 14 & MUSM-ENT 0514112 & 3 & cocholla 1 & Maynas & Rio Curaray & & 162 & $1^{13} 31^{1} 46.2^{\prime \prime}$ & $75^{\circ} 25^{\prime} 30.4 "$ & & & & W. Paredes & Etiqueta & Consultoría EIA & Gonyleptoidea & Cosmetidae & $\begin{array}{l}\text { M. Medrano y A. Ayala-Apaza, } \\
2021\end{array}$ \\
\hline 15 & MUSM-ENT 0512476 & 1 & Cocholla 2 & Maynas & Río Nashiño & & 190 & $1^{12} 23^{\prime} 25.7^{\prime \prime}$ & $75^{\circ} 16^{\prime} 46.64 "$ & 25 & xii & 2008 & R. Yawarcani & Etiqueta & Consultoría EIA & Gonyleptoidea & Cosmetidae & $\begin{array}{l}\text { M. Medrano y A. Ayala-Apaza, } \\
2021\end{array}$ \\
\hline 16 & MUSM-ENT 0512016 & 1 & Cocholla simoni Roewer, $1928^{*}$ & Loreto & Río Samiria & Cocha Shinguito & 90 & $5^{\circ} 10^{\prime}$ & $7^{7} 39^{\prime}$ & & $\mathrm{vi}$ & 1990 & T. Erwin & Etiqueta & $\begin{array}{l}\text { MHN-UNMSM/Smith- } \\
\text { sonian Inst. }\end{array}$ & Gonyleptoidea & Cosmetidae & A. Ayala-Apaza, 2021 \\
\hline 17 & MUSM-ENT 0512198 & 1 & Cocholla simoni Roewer, $1928^{*}$ & Loreto & Río Samiria & Cocha Shinguito & 90 & $5^{\circ} 10^{\prime}$ & 74'39' & & v & 1990 & T. Erwin & Etiqueta & $\begin{array}{l}\text { MHN-UNMSM/Smith- } \\
\text { sonian Inst. }\end{array}$ & Gonyleptoidea & Cosmetidae & A. Ayala-Apaza, 2021 \\
\hline 18 & MUSM-ENT 0512381 & 1 & Cocholla simoni Roewer, 1928* & Loreto & Río Samiria & Pithecia & 111 & $5^{\circ} 10^{\prime}$ & $7^{7 \circ} 39^{\prime}$ & 15 & viii & 1989 & D. Silva & Etiqueta & $\begin{array}{l}\text { MHN-UNMSM/Smith- } \\
\text { sonian Inst. }\end{array}$ & Gonyleptoidea & Cosmetidae & A. Ayala-Apaza, 2021 \\
\hline 19 & MUSM-ENT 0512383 & 1 & Cocholla simoni Roewer, 1928* & Ucayali & Bajo Inahuaillo & Campamento Venado & 500 & $06^{\circ} 51$ & $75^{\circ} 06^{\prime}$ & 14 & vii & 1988 & R. Fernandez & $\begin{array}{l}\text { Guizado \& } \\
\text { Girard } 1966 \\
\end{array}$ & MHN-UNMSM & Gonyleptoidea & Cosmetidae & A. Ayala-Apaza, 2021 \\
\hline 20 & MUSM-ENT 0512394 & 2 & Cocholla simoni Roewer, 1928* & Loreto & Río Samiria & P.V. Boca del Samiria & & $4^{\circ} 41^{\prime}$ & $74^{\circ} 20^{\prime}$ & & viii & 1989 & D. Silva & Etiqueta & $\begin{array}{l}\text { MHN-UNMSM/Smith- } \\
\text { sonian Inst. }\end{array}$ & Gonyleptoidea & Cosmetidae & A. Ayala-Apaza, 2021 \\
\hline 21 & MUSM-ENT 0512396 & 1 & Cocholla simoni Roewer, 1928* & Requena & Jenaro Herrera & $\begin{array}{l}\text { Centro de Investigación } \\
\text { Jenaro Herrera }\end{array}$ & 100 & $04^{\circ} 55^{\prime}$ & $73^{\circ} 45^{\prime}$ & 25 & viii & 1988 & D. Silva & Etiqueta & MHN-UNMSM & Gonyleptoidea & Cosmetidae & A. Ayala-Apaza, 2021 \\
\hline 22 & MUSM-ENT 0512429 & 2 & Cocholla simoni Roewer, 1928* & Loreto & Río Samiria & Cocha Shinguito & 90 & $5^{\circ} 10^{\prime}$ & 74³9' & & iv-v & 1990 & T. Erwin & Etiqueta & $\begin{array}{l}\text { MHN-UNMSM/Smith- } \\
\text { sonian Inst. }\end{array}$ & Gonyleptoidea & Cosmetidae & A. Ayala-Apaza, 2021 \\
\hline 23 & MUSM-ENT 0512440 & 1 & Cocholla simoni Roewer, $1928^{*}$ & Maynas & Río Napo & Terraza media plana & 190 & $1^{\circ} 23^{\prime} 39.36 "$ & $75^{\circ} 13^{\prime} 51.86^{\prime \prime}$ & & $\mathrm{i}$ & 2009 & R. Yawarcani & Etiqueta & Consultoría EIA & Gonyleptoidea & Cosmetidae & A. Ayala-Apaza, 2021 \\
\hline 24 & MUSM-ENT 0512499 & 1 & Cocholla simoni Roewer, 1928* & Loreto & Río Samiria & Cocha Shinguito & 90 & $5^{\circ} 10^{\prime}$ & 74³9' & 30 & v & 1990 & T. Erwin & Etiqueta & $\begin{array}{l}\text { MHN-UNMSM/Smith- } \\
\text { sonian Inst. }\end{array}$ & Gonyleptoidea & Cosmetidae & A. Ayala-Apaza, 2021 \\
\hline 25 & MUSM-ENT 0512564 & 1 & Cocholla simoni Roewer, 1928* & Loreto & Río Samiria & P.V. Boca del Samiria & & $4^{\circ} 41^{\prime}$ & $74^{\circ} 20^{\prime}$ & 11 & viii & 1989 & D. Silva & Etiqueta & $\begin{array}{l}\text { MHN-UNMSM/Smith- } \\
\text { sonian Inst. }\end{array}$ & Gonyleptoidea & Cosmetidae & A. Ayala-Apaza, 2021 \\
\hline 28 & MUSM-ENT 0512579 & 1 & Cocholla simoni Roewer, 1928* & Loreto & Río Samiria & PV. Boca del Samiria & & $4^{\circ} 41^{\prime}$ & $74^{\circ} 20^{\prime}$ & 11 & viii : & 1989 & D. Silva & Etiqueta & $\begin{array}{l}\text { MHN-UNMSM/Smith- } \\
\text { sonian Inst. }\end{array}$ & Gonyleptoidea & Cosmetidae & A. Ayala-Apaza, 2021 \\
\hline
\end{tabular}




\begin{tabular}{|c|c|c|c|c|c|c|c|c|c|c|c|c|c|c|c|c|c|c|}
\hline$\#$ & Código de ingreso & No. Ind. & 1. Especie/Morfoespecie & Provincia & Localidad & Lugar de colecta & Altitud & Latitud & Longitud & Dia & Mes & Año & colectores & $\begin{array}{l}\text { Fuente para } \\
\text { geooreferen- } \\
\text { ciación }\end{array}$ & Proyecto de colecta & Superfamilia & Familia & Identificación \\
\hline 29 & MUSM-ENT 0514086 & 1 & Cocholla simoni Roewer, $1928^{*}$ & Loreto & Rio Samiria & Pithecia & 111 & $5^{\circ} 10^{\prime}$ & $74^{\circ} 39^{\prime}$ & 16 & viii & 1989 & D. Silva & Etiqueta & $\begin{array}{l}\text { MHN-UNMSM/Smith- } \\
\text { sonian Inst. }\end{array}$ & Gonyleptoidea & Cosmetidae & A. Ayala-Apaza, 2021 \\
\hline 30 & MUSM-ENT 0514102 & 2 & Cocholla simoni Roewer, $1928^{*}$ & Loreto & Río Copalyacu & HBH Varillal & 136 & $03^{\circ} 45^{\prime} 15.36^{\prime \prime}$ & $75^{\circ} 22^{\prime} 14.9^{\prime \prime}$ & 24 & iii & 2008 & W. Paredes & Etiqueta & Consultoría EIA & Gonyleptoidea & Cosmetidae & A. Ayala-Apaza, 2021 \\
\hline 31 & MUSM-ENT 0514104 & 2 & Cocholla simoni Roewer, $1928^{*}$ & Maynas & Río Napo & & 190 & $0^{\circ} 58^{\prime} 48.80 "$ & $75^{\circ} 13^{2} 27.69^{\prime \prime}$ & 11 & i & 2009 & R. Yawarcani & Etiqueta & Consultoría EIA & Gonyleptoidea & Cosmetidae & A. Ayala-Apaza, 2021 \\
\hline 32 & MUSM-ENT 0514109 & 5 & Cocholla simoni Roewer, $1928^{*}$ & Loreto & Río Samiria & Cocha Shinguito & 90 & $5^{\circ} 10^{\prime}$ & $74^{\circ} 39^{\prime}$ & & $i v-v$ & 1990 & Erwin, Silva et al. & Etiqueta & $\begin{array}{l}\text { MHN-UNMSM/Smith- } \\
\text { sonian Inst. }\end{array}$ & Gonyleptoidea & Cosmetidae & A. Ayala-Apaza, 2021 \\
\hline 33 & MUSM-ENT 0512010 & 1 & Cosmetidae 10 & Loreto & Río Samiria & Cocha Shinguito & 90 & $5^{\circ} 10^{\prime}$ & $74^{\circ} 39^{\prime}$ & & v-vi & 1990 & T. Erwin & Etiqueta & $\begin{array}{l}\text { MHN-UNMSM/Smith- } \\
\text { sonian Inst. }\end{array}$ & Gonyleptoidea & Cosmetidae & A. Ayala-Apaza, 2021 \\
\hline 34 & MUSM-ENT 0512015 & 6 & Cosmetidae 10 & Loreto & Rio Samiria & Cocha Shinguito & 90 & $5^{\circ} 10^{\prime}$ & $74^{\circ} 39^{\prime}$ & & v-vi & 1990 & T. Erwin & Etiqueta & $\begin{array}{l}\text { MHN-UNMSM/Smith- } \\
\text { sonian Inst. }\end{array}$ & Gonyleptoidea & Cosmetidae & A. Ayala-Apaza, 2021 \\
\hline 35 & MUSM-ENT 0512202 & 4 & Cosmetidae 10 & Loreto & Río Samiria & Cocha Shinguito & 90 & $5^{\circ} 10^{\prime}$ & 74"39' & 13 & v & 1990 & T. Erwin & Etiqueta & $\begin{array}{l}\text { MHN-UNMSM/Smith- } \\
\text { sonian Inst. }\end{array}$ & Gonyleptoidea & Cosmetidae & A. Ayala-Apaza, 2021 \\
\hline 36 & MUSM-ENT 0512242 & 1 & Cosmetidae 10 & Loreto & Río Samiria & Cocha Shinguito & 90 & $5^{\circ} 10^{\prime}$ & $74^{\circ} 39^{\prime}$ & 12 & v & 1990 & T. Erwin & Etiqueta & $\begin{array}{l}\text { MHN-UNMSM/Smith- } \\
\text { sonian Inst. }\end{array}$ & Gonyleptoidea & Cosmetidae & A. Ayala-Apaza, 2021 \\
\hline 37 & MUSM-ENT 0512364 & 2 & Cosmetidae 10 & Loreto & Río Samiria & Cocha Shinguito & 90 & $5^{\circ} 10^{\prime}$ & $74^{\circ} 39^{\prime}$ & & v & 1990 & D. Silva & Etiqueta & $\begin{array}{l}\text { MHN-UNMSM/Smith- } \\
\text { sonian Inst. }\end{array}$ & Gonyleptoidea & Cosmetidae & A. Ayala-Apaza, 2021 \\
\hline 38 & MUSM-ENT 0512430 & 10 & Cosmetidae 10 & Loreto & Río Samiria & Cocha Shinguito & 90 & $5^{\circ} 10^{\prime}$ & $74^{\circ} 39^{\prime}$ & & $i v-v$ & 1990 & T. Erwin & Etiqueta & $\begin{array}{l}\text { MHN-UNMSM/Smith- } \\
\text { sonian Inst. }\end{array}$ & Gonyleptoidea & Cosmetidae & A. Ayala-Apaza, 2021 \\
\hline 39 & MUSM-ENT 0512488 & 2 & Cosmetidae 10 & Maynas & Río Napo & & 190 & $0^{\circ} 58^{\prime} 48.80^{\prime \prime}$ & $75^{\circ} 13^{\prime} 27.69^{\prime \prime}$ & 11 & $\mathrm{i}$ & 2009 & R. Yawarcani & Etiqueta & Consultoría EIA & Gonyleptoidea & Cosmetidae & A. Ayala-Apaza, 2021 \\
\hline 40 & MUSM-ENT 0512489 & 1 & Cosmetidae 10 & Loreto & Río Samiria & Cocha Shinguito & 90 & $5^{\circ} 10^{\prime}$ & $74^{\circ} 39^{\prime}$ & 23 & v & 1990 & T. Erwin & Etiqueta & $\begin{array}{l}\text { MHN-UNMSM/Smith- } \\
\text { sonian Inst. }\end{array}$ & Gonyleptoidea & Cosmetidae & A. Ayala-Apaza, 2021 \\
\hline 41 & MUSM-ENT 0512541 & 2 & Cosmetidae 10 & Loreto & Río Samiria & Cocha Shinguito & 90 & $5^{\circ} 10^{\prime}$ & $74^{\circ} 39^{\prime}$ & 30 & v & 1990 & T. Erwin & Etiqueta & $\begin{array}{l}\text { MHN-UNMSM/Smith- } \\
\text { sonian IIst. }\end{array}$ & Gonyleptoidea & Cosmetidae & A. Ayala-Apaza, 2021 \\
\hline 42 & MUSM-ENT 0512546 & 16 & Cosmetidae 10 & Loreto & Río Samiria & Cocha Shinguito & 90 & $5^{\circ} 10^{\prime}$ & $74^{\circ} 39^{\prime}$ & & v-vi & 1990 & T. Erwin & Etiqueta & $\begin{array}{l}\text { MHN-UNMSM/Smith- } \\
\text { sonian Inst. }\end{array}$ & Gonyleptoidea & Cosmetidae & A. Ayala-Apaza, 2021 \\
\hline 43 & MUSM-ENT 0512558 & 10 & Cosmetidae 10 & Loreto & Río Samiria & Cocha Shinguito & 90 & $5^{\circ} 10^{\prime}$ & $74^{\circ} 39^{\prime}$ & 20 & v & 1990 & D. Silva & Etiqueta & $\begin{array}{l}\text { MHN-UNMSM/Smith- } \\
\text { sonian IIst. }\end{array}$ & Gonyleptoidea & Cosmetidae & A. Ayala-Apaza, 2021 \\
\hline 44 & MUSM-ENT 0512576 & 1 & Cosmetidae 10 & Loreto & Río Samiria & Cocha Shinguito & 90 & $5^{\circ} 10^{\prime}$ & $74^{\circ} 39^{\prime}$ & & $i v-v$ & 1990 & T. Erwin & Etiqueta & $\begin{array}{l}\text { MHN-UNMSM/Smith- } \\
\text { sonian Inst. }\end{array}$ & Gonyleptoidea & Cosmetidae & A. Ayala-Apaza, 2021 \\
\hline 45 & MUSM-ENT 0514092 & 2 & Cosmetidae 10 & Loreto & Río Samiria & Cocha Shinguito & 90 & $5^{\circ} 10^{\prime}$ & $74^{\circ} 39^{\prime}$ & & v & 1990 & D. Silva & Etiqueta & $\begin{array}{l}\text { MHN-UNMSM/Smith- } \\
\text { sonian Inst. }\end{array}$ & Gonyleptoidea & Cosmetidae & A. Ayala-Apaza, 2021 \\
\hline 46 & MUSM-ENT 0514095 & 14 & Cosmetidae 10 & Loreto & Río Samiria & Cocha Shinguito & 90 & $5^{\circ} 10^{\prime}$ & $74^{\circ} 39^{\prime}$ & 24 & vi & 1990 & T. Erwin & Etiqueta & $\begin{array}{l}\text { MHN-UNMSM/Smith- } \\
\text { sonian Inst. }\end{array}$ & Gonyleptoidea & Cosmetidae & A. Ayala-Apaza, 2021 \\
\hline 47 & MUSM-ENT 0514105 & 1 & Cosmetidae 10 & Maynas & Rio Napo & & 190 & $0^{\circ} 58^{\prime} 48.80 "$ & $75^{\circ} 13^{\prime 2} 27.69^{\prime \prime}$ & 11 & $\mathrm{i}$ & 2009 & R. Yawarcani & Etiqueta & Consultoría EIA & Gonyleptoidea & Cosmetidae & A. Ayala-Apaza, 2021 \\
\hline 48 & MUSM-ENT 0514510 & 1 & Cosmetidae 10 & Loreto & Río Samiria & Cocha Shinguito & 90 & $5^{\circ} 10^{\prime}$ & $74^{\circ} 39^{\prime}$ & 24 & vi & 1990 & T. Erwin & Etiqueta & $\begin{array}{l}\text { MHN-UNMSM/Smith- } \\
\text { sonian Inst. }\end{array}$ & Gonyleptoidea & Cosmetidae & $\begin{array}{l}\text { A. Ayala-Apaza y M. Medrano } \\
2021\end{array}$ \\
\hline 49 & MUSM-ENT 0514501 & 12 & Cosmetidae 10 & Loreto & Río Samiria & Cocha Shinguito & 90 & $5^{\circ} 10^{\prime}$ & $74^{\circ} 39^{\prime}$ & & vi & 1990 & T. Erwin & Etiqueta & $\begin{array}{l}\text { MHN-UNMSM/Smith- } \\
\text { sonian Inst. }\end{array}$ & Gonyleptoidea & Cosmetidae & A. Ayala-Apaza, 2021 \\
\hline 50 & MUSM-ENT 0512196 & 1 & Cosmetidae 11 & Maynas & Río Curaray & Napo & & $1^{\circ} 35^{\prime} 16.1^{\prime \prime}$ & $75^{\circ} 24^{\prime} 19.7^{\prime \prime}$ & 12 & $\mathrm{xi}$ & 2007 & . Ramírez & Etiqueta & Consultoría EIA & Gonyleptoidea & Cosmetidae & A. Ayala-Apaza, 2021 \\
\hline 51 & MUSM-ENT 0512347 & 2 & Cosmetidae 11 & Maynas & Río Napo & & 210 & $1^{\circ} 45^{\prime} 23.44^{\prime \prime}$ & $34^{\circ} 43^{\prime 22.93 "}$ & 22 & xii & 2008 & W. Yawarcani & Etiqueta & Consultoría EIA & Gonyleptoidea & Cosmetidae & A. Ayala-Apaza, 2021 \\
\hline 52 & MUSM-ENT 0514099 & 1 & Cosmetidae 11 & Maynas & Río Napo & & 190 & $0^{\circ} 58^{\prime} 48.80^{\prime \prime}$ & $75^{\circ} 13^{\prime} 27.69^{\prime \prime}$ & 11 & $\mathrm{i}$ & 2009 & R. Yawarcani & Etiqueta & Consultoría EIA & Gonyleptoidea & Cosmetidae & A. Ayala-Apaza, 2021 \\
\hline 53 & MUSM-ENT 0512184 & 1 & Cosmetidae 13 & Maynas & Río Napo & & 194 & $1^{\circ} 51^{\prime \prime 42.5 "}$ & $75^{\circ} 24^{\prime} 06.1^{\prime \prime}$ & $23-24$ & ii & 2008 & W. Paredes & Etiqueta & Consultoría EIA & Gonyleptoidea & Cosmetidae & A. Ayala-Apaza, 2021 \\
\hline 54 & MUSM-ENT 0514097 & 1 & Cosmetidae 13 & Maynas & Río Curaray & & 209 & $1^{\circ} 56^{\prime} 5.7 "$ & $75^{\circ} 22^{\prime} 20.8^{\prime \prime}$ & 10 & ii & 2008 & W. Paredes & Etiqueta & Consultoría EIA & Gonyleptoidea & Cosmetidae & A. Ayala-Apaza, 2021 \\
\hline 55 & MUSM-ENT 0512371 & 2 & Cosmetidae 14 & Loreto & Río Samiria & Pithecia & 111 & $5^{\circ} 10^{\prime}$ & $74^{\circ} 39^{\prime}$ & 16 & viii & 1989 & D. Silva & Etiqueta & $\begin{array}{l}\text { MHN-UNMSM/Smith- } \\
\text { sonian Inst. }\end{array}$ & Gonyleptoidea & Cosmetidae & A. Ayala-Apaza, 2021 \\
\hline 56 & MUSM-ENT 0512435 & 5 & Cosmetidae 14 & Loreto & Rio Samiria & Cocha Shinguito & 90 & $5^{\circ} 10^{\prime}$ & $74^{\circ} 39^{\prime}$ & 11 & v & 1990 & T. Erwin & Etiqueta & $\begin{array}{l}\text { MHN-UNMSM/Smith- } \\
\text { sonian Inst. }\end{array}$ & Gonyleptoidea & Cosmetidae & A. Ayala-Apaza, 2021 \\
\hline 57 & MUSM-ENT 0514087 & 1 & Cosmetidae 15 & Maynas & Rio Napo & & 200 & $1^{\circ} 16^{\prime} 40.62^{\prime \prime}$ & $75^{\circ} 17^{\prime 1} 18.96^{\prime \prime}$ & 28 & xii & 2008 & R. Yawarcani & Etiqueta & Consultoría EIA & Gonyleptoidea & Cosmetidae & A. Ayala-Apaza, 2021 \\
\hline 58 & MUSM-ENT 0512431 & 1 & Cosmetidae 2 & Loreto & Rio Samiria & $\begin{array}{l}\text { Cocha Shinguito, bajo } \\
\text { tronco caido }\end{array}$ & 90 & $5^{\circ} 10^{\prime}$ & $74^{\circ} 39^{\prime}$ & 27 & v & 1990 & T. Erwin & Etiqueta & $\begin{array}{l}\text { MHN-UNMSM/Smith- } \\
\text { sonian Inst. }\end{array}$ & Gonyleptoidea & Cosmetidae & A. Ayala-Apaza, 2021 \\
\hline
\end{tabular}




\begin{tabular}{|c|c|c|c|c|c|c|c|c|c|c|c|c|c|c|c|c|c|c|}
\hline$\#$ & Código de ingreso & No. Ind. & 4. Especie/Morfoespecie & Provincia & Localidad & Lugar de colecta & Altitu & d Latitud & Longitud & Dia & Mes & Año & colectores & $\begin{array}{l}\text { Fuente para } \\
\text { georeferen- } \\
\text { ciación }\end{array}$ & Proyecto de colecta & Superfamilia & Familia & Identificación \\
\hline 59 & MUSM-ENT 0512436 & 1 & Cosmetidae 2 & Loreto & Río Samiria & Cocha Shinguito & 90 & $5^{\circ} 10^{\prime}$ & $74^{\circ} 39^{\prime}$ & & $i v-v$ & 1990 & T. Erwin & Etiqueta & $\begin{array}{l}\text { MHN-UNMSM/Smith- } \\
\text { sonian Inst. }\end{array}$ & Gonyleptoidea & Cosmetidae & A. Ayala-Apaza, 2021 \\
\hline 60 & MUSM-ENT 0512565 & 1 & Cosmetidae 2 & Requena & Jenaro Herrera & $\begin{array}{l}\text { Centro de Investigación } \\
\text { Jenaro terrera, en pal- } \\
\text { meras Astrocarium sp. }\end{array}$ & 100 & $04^{\circ} 55^{\prime}$ & $73^{\circ} 45^{\prime}$ & 23 & viii & 1988 & D. Silva & Etiqueta & MHN-UNMSM & Gonyleptoidea & Cosmetidae & A. Ayala-Apaza, 2021 \\
\hline 61 & MUSM-ENT 0514498 & 1 & Cosmetidae 2 & Loreto & Río Samiria & Cocha Shinguito & 90 & $5^{\circ} 10^{\prime}$ & $74^{\circ} 39^{\prime}$ & 12 & v & 1990 & T. Erwin & Etiqueta & $\begin{array}{l}\text { MHN-UNMSM/Smith- } \\
\text { sonian Inst. }\end{array}$ & Gonyleptoidea & Cosmetidae & A. Ayala-Apaza, 2021 \\
\hline 62 & MUSM-ENT 0512142 & 1 & Cosmetidae 21 & Loreto & Rio Samiria & P.V. Boca del Samiria & & $4^{\circ} 41^{\prime}$ & $74^{\circ} 20^{\prime}$ & 11 & viii & 1989 & D. Silva & Etiqueta & $\begin{array}{l}\text { MHN-UNMSM/Smith- } \\
\text { sonian IIst. }\end{array}$ & Gonyleptoidea & Cosmetidae & $\begin{array}{l}\text { A. Avala-Apaza y M. Medrano, } \\
2021\end{array}$ \\
\hline 63 & MUSM-ENT 0512426 & 1 & Cosmetidae 21 & Loreto & Río Samiria & Cocha Shinguito & 90 & $5^{\circ} 10^{\prime}$ & $74^{\circ} 39^{\prime}$ & 10 & $v$ & 1990 & T. Erwin & Etiqueta & $\begin{array}{l}\text { MHN-UNMSM/Smith- } \\
\text { sonian IIst. }\end{array}$ & Gonyleptoidea & Cosmetidae & A. Ayala-Apaza, 2021 \\
\hline 64 & MUSM-ENT 0512443 & 3 & Cosmetidae 21 & Loreto & Rio Samiria & $\begin{array}{l}\text { Cocha Shinguito, bajo } \\
\text { tronco caido }\end{array}$ & 90 & $5^{\circ} 10^{\prime}$ & $74^{\circ} 39^{\prime}$ & 27 & $\mathrm{v}$ & 1990 & T. Erwin & Etiqueta & $\begin{array}{l}\text { MHN-UNMSM/Smith- } \\
\text { sonian Inst. }\end{array}$ & Gonyleptoidea & Cosmetidae & A. Ayala-Apaza, 2021 \\
\hline 65 & MUSM-ENT 0512577 & 2 & Cosmetidae 21 & Loreto & Rio Samiria & Pithecia & 111 & $5^{\circ} 10^{\prime}$ & $74^{\circ} 39^{\prime}$ & 16 & viii & 1989 & D. Silva & Etiqueta & $\begin{array}{l}\text { MHN-UNMSM/Smith- } \\
\text { sonian Inst. }\end{array}$ & Gonyleptoidea & Cosmetidae & A. Ayala-Apaza, 2021 \\
\hline 66 & MUSM-ENT 0512597 & 1 & Cosmetidae 21 & Loreto & Rio Samiria & Cocha Shinguito & 90 & $5^{\circ} 10^{\prime}$ & $74^{\circ} 39^{\prime}$ & 10 & v & 1990 & T. Erwin & Etiqueta & $\begin{array}{l}\text { MHN-UNMSM/Smith- } \\
\text { sonian Inst. }\end{array}$ & Gonyleptoidea & Cosmetidae & A. Ayala-Apaza, 2021 \\
\hline 67 & MUSM-ENT 0514117 & 1 & Cosmetidae 21 & Loreto & Rio Samiria & Cocha Shinguito & 90 & $5^{\circ} 10^{\prime}$ & $74^{\circ} 39^{\prime}$ & 14 & v & 1990 & Erwin, Silva et al. & Etiqueta & $\begin{array}{l}\text { MHN-UNMSM/Smith- } \\
\text { sonian Inst. }\end{array}$ & Gonyleptoidea & Cosmetidae & A. Ayala-Apaza, 2021 \\
\hline 68 & MUSM-ENT 0512033 & 1 & Cosmetidae 24 & Loreto & Río Napo & Napo & 176 & $1^{\circ 5} 1^{4} 42.5^{\prime \prime}$ & $75^{\circ} 24^{\prime} 06.1^{\prime \prime}$ & $24-25$ & ii & 2008 & W. Paredes & Etiqueta & Consultoría EIA & Gonyleptoidea & Cosmetidae & $\begin{array}{l}\text { A. Ayala-Apaza y M. Medrano, } \\
2021\end{array}$ \\
\hline 69 & MUSM-ENT 0512088 & 8 & Cosmetidae 9 & Maynas & Rio Curaray & & 209 & $1^{\circ} 56^{\prime} 5.7 "$ & $75^{\circ} 22^{\prime} 20.8^{\prime \prime}$ & 10 & ii & 2008 & W. Paredes & Etiqueta & Consultoría EIA & Gonyleptoidea & Cosmetidae & A. Ayala-Apaza, 2021 \\
\hline 70 & MUSM-ENT 0512185 & 1 & Cosmetidae 9 & Maynas & Río Napo & & 194 & $1^{\circ} 51^{\prime} 42.5^{\prime \prime}$ & $75^{\circ} 24^{\prime} 06.1^{\prime \prime}$ & $23-24$ & ii & 2008 & W. Paredes & Etiqueta & Consultoría EIA & Gonyleptoidea & Cosmetidae & A. Ayala-Apaza, 2021 \\
\hline 71 & MUSM-ENT 0512362 & 3 & Cosmetidae 9 & Maynas & Río Napo & & 210 & $1^{\circ} 45^{\prime 23.44 "}$ & $34^{\circ} 43^{\prime} 22.93^{\prime \prime}$ & 22 & xii & 2008 & w. Yawarcani & Etiqueta & Consultoría EIA & Gonyleptoidea & Cosmetidae & A. Ayala-Apaza, 2021 \\
\hline 72 & MUSM-ENT 0512486 & 2 & Cosmetidae 9 & Maynas & Río Napo & & 195 & ${ }^{\circ} 52^{2} 34.277^{\prime \prime}$ & $74^{\circ} 47^{\prime} 33.27^{\prime \prime}$ & 10 & $\mathrm{i}$ & 2009 & R. Yawarcani & Etiqueta & Consultoría EIA & Gonyleptoidea & Cosmetidae & A. Ayala-Apaza, 2021 \\
\hline 73 & MUSM-ENT 0512487 & 2 & Cosmetidae 9 & Maynas & Río Napo & & 190 & $0^{\circ} 58^{\prime} 48.80^{\prime \prime}$ & $75^{\circ} 13^{\prime} 27.69^{\prime \prime}$ & 11 & $\mathrm{i}$ & 2009 & R. Yawarcani & Etiqueta & Consultoría EIA & Gonyleptoidea & Cosmetidae & A. Ayala-Apaza, 2021 \\
\hline 74 & MUSM-ENT 0512615 & 1 & Cosmetidae 9 & Maynas & Río Curaray & Napo, colinas medias & 178 & $1^{\circ} 53^{\prime} 15.5^{\prime \prime}$ & $75^{\circ} 21^{\prime} 51.0^{\prime \prime}$ & 19 & ii & 2008 & W. Paredes & Etiqueta & Consultoría EIA & Gonyleptoidea & Cosmetidae & A. Ayala-Apaza, 2021 \\
\hline 75 & MUSM-ENT 0514098 & 2 & Cosmetidae 9 & Maynas & Río Curaray & & 209 & $1^{\circ} 56^{\prime} 5.7 "$ & $75^{\circ} 22^{\prime} 20.8^{\prime \prime}$ & 10 & ii & 2008 & W. Paredes & Etiqueta & Consultoría EIA & Gonyleptoidea & Cosmetidae & A. Ayala-Apaza, 2021 \\
\hline 76 & MUSM-ENT 0514107 & 3 & Cosmetidae 9 & Maynas & Río Napo & & 190 & $1^{\circ} 12 ' 33.8^{\prime \prime}$ & $75^{\circ} 22^{\prime} 32^{\prime \prime}$ & 2 & $\mathrm{i}$ & 2009 & R. Yawarcani & Etiqueta & Consultoría EIA & Gonyleptoidea & Cosmetidae & A. Ayala-Apaza, 2021 \\
\hline 77 & MUSM-ENT 0513834 & 1 & Cosmetidae aff. 10 & Loreto & Trompeteros & & 143 & $3^{\circ} 49^{\prime} 37.14^{\prime \prime}$ & $75^{\circ} 3^{\prime} 10.82^{\prime \prime}$ & $6-7$ & iv & 2018 & T. Neyra & Etiqueta & Consultoría EIA & Gonyleptoidea & Cosmetidae & A. Ayala-Apaza, 2021 \\
\hline 78 & MUSM-ENT 0514506 & 1 & Cranaidae gen.n. & Maynas & Río Curaray & & 209 & $1^{\circ} 56^{\prime} 5.7 "$ & $75^{\circ} 22^{\prime} 20.8^{\prime \prime}$ & 10 & ii & 2008 & W. Paredes & Etiqueta & Consultoría EIA & Gonyleptoidea & Cranaidae & $\begin{array}{l}\text { O. Villarreal-Manzanilla y A. } \\
\text { Avalla-Apaza, } 2021\end{array}$ \\
\hline 79 & MUSM-ENT 0512382 & 3 & Cynorta marginalis Banks, 1909* & Requena & Jenaro Herrera & $\begin{array}{l}\text { Centro de Investigación } \\
\text { Jenaro terrera, en pal- } \\
\text { meras Astrocarium sp. }\end{array}$ & 400 & $04^{\circ} 55^{\prime}$ & $73^{\circ} 45^{\prime}$ & 24 & viii & 1988 & D. Silva & Etiqueta & MHN-UNMSM & Gonyleptoidea & Cosmetidae & A. Ayala-Apaza, 2021 \\
\hline 80 & MUSM-ENT 0512455 & 7 & Cynorta marginalis Banks, 1909* & Requena & Jenaro Herrera & $\begin{array}{l}\text { Centro de Investigación } \\
\text { Jenaro Herrera }\end{array}$ & 500 & $04^{\circ} 55^{\prime}$ & $73^{\circ} 45^{\prime}$ & 26 & viii & 1988 & D. Silva & Etiqueta & MHN-UNMSM & Gonyleptoidea & Cosmetidae & A. Ayala-Apaza, 2021 \\
\hline 81 & MUSM-ENT 0514474 & 3 & Cynorta marginalis Banks, 1909* & Maynas & Rio Itaya & Quebrada Yanayacu & 102 & $04^{\circ} 16^{\prime} 49.9^{\prime \prime}$ & $73^{\circ} 40^{\prime} 52.2^{\prime \prime}$ & $12-20$ & vii & 2012 & D. Silva & Etiqueta & MHN-UNMSM & Gonyleptoidea & Cosmetidae & A. Ayala-Apaza, 2021 \\
\hline 82 & MUSM-ENT 0512140 & 5 & Discosomaticus cinctus (Perty, 1833)* & Requena & Jenaro Herrera & $\begin{array}{l}\text { Centro de Investigación } \\
\text { Elenaro Herrera }\end{array}$ & 100 & $04^{\circ} 55^{\prime}$ & $73^{\circ} 45^{\prime}$ & 25 & viii & 1988 & D. Silva & Etiqueta & MHN-UNMSM & Gonyleptoidea & Cosmetidae & A. Ayala-Apaza, 2021 \\
\hline 83 & MUSM-ENT 0512469 & 11 & Discosomaticus cinctus (Perty, 1833)* & Requena & Jenaro Herrera & $\begin{array}{l}\text { Centro de Investigación } \\
\text { Jenaro Herrera }\end{array}$ & 500 & $04^{\circ} 55^{\prime}$ & $73^{\circ} 45^{\prime}$ & 25 & viii & 1988 & D. Silva & Etiqueta & MHN-UNMSM & Gonyleptoidea & Cosmetidae & M. Medrano, 2021 \\
\hline 84 & MUSM-ENT 0512573 & 1 & Discosomaticus cinctus (Perty, 1833)* & Requena & Jenaro Herrera & $\mathrm{km} 4.5 \mathrm{JH}$-Pto Angamos & & $0^{\circ} 55^{\prime}$ & $73^{\circ} 45^{\prime}$ & 21 & viii & 1990 & P. Lozada & Google Earth & TMHN-UNMSM & Gonyleptoidea & Cosmetidae & A. Ayala-Apaza, 2021 \\
\hline 85 & MUSM-ENT 0512014 & 4 & Eulibitia aff. pollux Medrano \& Kury, 2017 & Loreto & Rio Samiria & Cocha Shinguito & 90 & $5^{\circ} 10^{\prime}$ & $74^{\circ} 39^{\prime}$ & & $v-v i$ & 1990 & T. Erwin & Etiqueta & $\begin{array}{l}\text { MHN-UNMSM/Smith- } \\
\text { sonian Inst. }\end{array}$ & Gonyleptoidea & Cosmetidae & A. Ayala-Apaza, 2021 \\
\hline 86 & MUSM-ENT 0512360 & 9 & Eulibitia aff. pollux Medrano \& Kury, 2017 & Loreto & Rio Samiria & Cocha Shinguito & 90 & $5^{\circ} 10^{\prime}$ & $74^{\circ} 39^{\prime}$ & & v & 1990 & T. Erwin & Etiqueta & $\begin{array}{l}\text { MHN-UNMSM/Smith- } \\
\text { sonian Inst. }\end{array}$ & Gonyleptoidea & Cosmetidae & A. Ayala-Apaza, 2021 \\
\hline 87 & MUSM-ENT 0512524 & 4 & Eulibitia aff. pollux Medrano \& Kury, 2017 & Loreto & Rio Samiria & Cocha Shinguito & 90 & $5^{\circ} 10^{\prime}$ & $74^{\circ} 39^{\prime}$ & 17 & v & 1990 & T. Erwin & Etiqueta & $\begin{array}{l}\text { MHN-UNMSM/Smith- } \\
\text { sonian Inst. }\end{array}$ & Gonyleptoidea & Cosmetidae & A. Ayala-Apaza, 2021 \\
\hline 88 & MUSM-ENT 0512551 & 2 & Eulibitia aff. pollux Medrano \& Kury, 2017 & Loreto & Rio Samiria & Cocha Shinguito & 90 & $5^{\circ} 10^{\prime}$ & $74^{\circ} 39^{\prime}$ & & & & T. Erwin & Etiqueta & $\begin{array}{l}\text { MHN-UNMSM/Smith- } \\
\text { sonian Inst. }\end{array}$ & Gonyleptoidea & Cosmetidae & A. Ayala-Apaza, 2021 \\
\hline
\end{tabular}




\begin{tabular}{|c|c|c|c|c|c|c|c|c|c|c|c|c|c|c|c|c|c|c|}
\hline$\#$ & Código de ingreso & No. Ind. & Especie/Morfoespecie & Provincia & Localidad & Lugar de colecta & $\begin{array}{c}\text { Altitud } \\
\mathrm{m}\end{array}$ & Latitud & Longitud & Dia & Mes & Año & colectores & $\begin{array}{l}\text { Fuente para } \\
\text { georeferen- } \\
\text { ciación }\end{array}$ & Proyecto de colecta & Superfamilia & Familia & Identificación \\
\hline 89 & MUSM-ENT 0512586 & 1 & Eulibitia aff. pollux Medrano \& Kury, 2017 & Loreto & Río Samiria & Cocha Shinguito & 90 & $5^{\circ} 10^{\prime}$ & $74^{\circ} 39^{\prime}$ & & $i v-v$ & 1990 & T. Erwin & Etiqueta & $\begin{array}{l}\text { MHN-UNMSM/Smith- } \\
\text { sonian Inst. }\end{array}$ & Gonyleptoidea & Cosmetidae & A. Ayala-Apaza, 2021 \\
\hline 90 & MUSM-ENT 0512779 & 3 & Eulibitia aff. pollux Medrano \& Kury, 2017 & Loreto & Río Samiria & Cocha Shinguito & 90 & $5^{\circ} 10^{\prime}$ & $74^{\circ} 39^{\prime}$ & 13 & v & 1990 & T. Erwin & Etiqueta & $\begin{array}{l}\text { MHN-UNMSM/Smith- } \\
\text { sonian Inst. }\end{array}$ & Gonyleptoidea & Cosmetidae & A. Ayala-Apaza, 2021 \\
\hline 91 & MUSM-ENT 0514088 & 4 & Eulibitia aff. pollux Medrano \& Kury, 2017 & Loreto & Río Samiria & Cocha Shinguito & 90 & $5^{\circ} 10^{\prime}$ & $74^{\circ} 39^{\prime}$ & 20 & v & 1990 & D. Silva & Etiqueta & $\begin{array}{l}\text { MHN-UNMSM/Smith- } \\
\text { sonian Inst. }\end{array}$ & Gonyleptoidea & cosmetidae & A. Ayala-Apaza, 2021 \\
\hline 92 & MUSM-ENT 0514094 & 4 & Eulibitia aff. pollux Medrano \& Kury, 2017 & Loreto & Río Samiria & Cocha Shinguito & 90 & $5^{\circ} 10^{\prime}$ & $74^{\circ} 39^{\prime}$ & 23 & v & 1990 & T. Erwin & Etiqueta & $\begin{array}{l}\text { MHN-UNMSM/Smith- } \\
\text { sonian Inst. }\end{array}$ & Gonyleptoidea & Cosmetidae & A. Ayala-Apaza, 2021 \\
\hline 93 & MUSM-ENT 0514500 & 1 & Eulibitia aff. pollux Medrano \& Kury, 2017 & Loreto & Río Samiria & Cocha Shinguito & 90 & $5^{\circ} 10^{\prime}$ & 74"39' & & vi & 1990 & T. Erwin & Etiqueta & $\begin{array}{l}\text { MHN-UNMSM/Smith- } \\
\text { sonian Inst. }\end{array}$ & Gonyleptoidea & Cosmetidae & A. Ayala-Apaza, 2021 \\
\hline 94 & MUSM-ENT 0507501 & 4 & Eutimesius simoni Roewer, 1913 & Requena & Jenaro Herrera & $\begin{array}{l}\text { Centro de Investigación } \\
\text { Jenaro Herrera }\end{array}$ & 100 & $04^{\circ} 55^{\prime}$ & $73^{\circ} 45^{\prime}$ & 24 & viii & 1988 & D. Silva & Etiqueta & MHN-UNMSM & Gonyleptoidea & Stygnidae & R. Pinto-da-Rocha, 1993 \\
\hline 95 & MUSM-ENT 0507502 & 1 & Eutimesius simoni Roewer, 1913 & Requena & Jenaro Herrera & $\begin{array}{l}\text { Centro de Investigación } \\
\text { Jenaro Herrera }\end{array}$ & 125 & $04^{\circ} 54^{\prime}$ & $73^{\circ} 40^{\prime}$ & 24 & viii & 1988 & D. Silva & Etiqueta & MHN-UNMSM & Gonyleptoidea & Stygnidae & A. Ayala-Apaza, 2018 \\
\hline 96 & MUSM-ENT 0507503 & 1 & Eutimesius simoni Roewer, 1913 & Requena & Jenaro Herrera & $\begin{array}{l}\text { Centro de Investigación } \\
\text { Jenaro Herrera }\end{array}$ & 100 & $04^{\circ} 55^{\prime}$ & $73^{\circ} 45^{\prime}$ & 24 & viii & 1988 & D. Silva & Etiqueta & MHN-UNMSM & Gonyleptoidea & Stygnidae & A. Ayala-Apaza, 2018 \\
\hline 97 & MUSM-ENT 0511847 & 1 & Eutimesius simoni Roewer, 1913 & Requena & Jenaro Herrera & $\begin{array}{l}\text { Purmat } 2 \text { años. km } 4.5 \\
\text { HH-Pto Angamos }\end{array}$ & & $04^{\circ} 55^{\prime}$ & $73^{\circ} 45^{\prime}$ & 28 & viii & 1990 & P. Lozada & Google Earth & & Gonyleptoidea & Stygnidae & A. Ayala-Apaza, 2018 \\
\hline 98 & MUSM-ENT 0512036 & 2 & Eutimesius simoni Roewer, 1913 & Maynas & Rio Napo & Rio Napo, colinas medias & 176 & $1^{\circ} 51^{\prime 42.5 "}$ & $75^{\circ} 24^{\prime} 06.1^{\prime \prime}$ & $24-27$ & ii & 2008 & W. Paredes & Etiqueta & Consultoría EIA & Gonyleptoidea & Stygnidae & A. Ayala-Apaza, 2018 \\
\hline 99 & MUSM-ENT 0512092 & 2 & Eutimesius simoni Roewer, 1913 & Maynas & Rio Curaray & & 209 & $1^{\circ} 56^{\prime} 5.7 "$ & $75^{\circ} 22^{\prime} 20.8^{\prime \prime}$ & 10 & ii & 2008 & W. Paredes & Etiqueta & Consultoría EIA & Gonyleptoidea & Stygnidae & A. Ayala-Apaza, 2018 \\
\hline 100 & MUSM-ENT 0513836 & 1 & Eutimesius simoni Roewer, 1913 & Loreto & Trompeteros & & 143 & $3^{\circ} 49^{\prime} 37.14^{\prime \prime}$ & $75^{\circ} 3^{\prime} 10.82^{\prime \prime}$ & $6-7$ & iv & 2018 & T. Neyra & Etiqueta & Consultoría EIA & Gonyleptoidea & Stygnidae & A. Ayala-Apaza, 2021 \\
\hline 101 & MUSM-ENT 0512003 & 2 & Gonyleptidae 1 & Maynas & Rio Nashiño & & 190 & $6^{\circ} 23^{3} 39.36^{\prime \prime}$ & $75^{\circ} 13^{\prime} 51.86^{\prime \prime}$ & 23 & xii & 2008 & R. Yawarcani & Etiqueta & Consultoría EIA & Gonyleptoidea & Gonyleptidae & A. Ayala-Apaza, 2021 \\
\hline 102 & MUSM-ENT 0512114 & 2 & Gonyleptidae 1 & Maynas & Río Napo & & 190 & 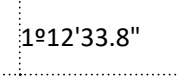 & 752''ㄹ" & 2 & $\mathrm{i}$ & 2009 & R. Yawarcani & Etiqueta & Consultoría EIA & Gonyleptoidea & Gonyleptidae & A. Ayala-Apaza, 2021 \\
\hline 103 & MUSM-ENT 0514503 & 1 & Gonyleptidae 1 & Maynas & Río Napo & Napo & 176 & $1^{\circ} 51^{\prime} 42.5^{\prime \prime}$ & $75^{\circ} 24^{\prime} 06.1^{\prime \prime}$ & $24-25$ & ii & 2008 & W. Paredes & Etiqueta & Consultoría EIA & Gonyleptoidea & Gonyleptidae & A. Ayala-Apaza, 2021 \\
\hline 104 & MUSM-ENT 0514505 & 1 & Gonyleptidae 2 & Maynas & Rio Curaray & & 209 & $1^{\circ} 56^{\prime} 5.7^{\prime \prime}$ & $75^{\circ} 22^{\prime} 20.8^{\prime \prime}$ & 10 & ii & 2008 & W. Paredes & Etiqueta & Consultoría EIA & Gonyleptoidea & Gonyleptidae & A. Ayala-Apaza, 2021 \\
\hline 105 & MUSM-ENT 0512157 & 3 & Gonyleptoidea 2 & Maynas & quitos & Laguna Quistococha & & $3^{\circ} 49^{4} 44.4^{\prime \prime}$ & $73^{\circ} 19^{\prime} 8.4^{\prime \prime}$ & 20 & vii & 1989 & D. Silva & Google Earth! & hMHN-UNMSM & Gonyleptoidea & & A. Ayala-Apaza, 2021 \\
\hline 106 & MUSM-ENT 0514113 & 1 & Gonyleptoidea 4 & Maynas & Río Curaray & & 162 & $1^{\circ} 31^{\prime} 46.2^{\prime \prime}$ & $75^{\circ} 25^{\prime} 30.4^{\prime \prime}$ & & & & W. Paredes & Etiqueta & Consultoría EIA & Gonyleptoidea & & A. Ayala-Apaza, 2021 \\
\hline 107 & MUSM-ENT 0511924 & 1 & Hutamaia plei Tourinho \& Mendes, 2014* & Requena & Jenaro Herrera & $\begin{array}{l}\text { Centro de Investigación } \\
\text { Jenaro Herrera }\end{array}$ & 500 & $04^{\circ} 55^{\prime}$ & $73^{\circ} 45^{\prime}$ & 25 & viii & 1988 & D. Silva & Etiqueta & MHN-UNMSM & Gonyleptoidea & Gonyleptidae & A. Ayala-Apaza, 2021 \\
\hline 108 & MUSM-ENT 0511932 & 1 & Hutamaia plei Tourinho \& Mendes, 2014* & Loreto & Río Samiria & Cocha Shinguito & 90 & $5^{\circ} 10^{\prime}$ & $74^{\circ} 39^{\prime}$ & 10 & v & 1990 & T. Erwin & Etiqueta & $\begin{array}{l}\text { MHN-UNMSM/Smith- } \\
\text { sonian Inst. }\end{array}$ & Gonyleptoidea & Gonyleptidae & A. Ayala-Apaza, 2021 \\
\hline 109 & MUSM-ENT 0511933 & 1 & Hutamaia plei Tourinho \& Mendes, 2014* & Loreto & Rio Samiria & Cocha Shinguito & 90 & $5^{\circ} 10^{\prime}$ & $74^{\circ} 39^{\prime}$ & 9 & v & 1990 & T. Erwin & Etiqueta & $\begin{array}{l}\text { MHN-UNMSM/Smith- } \\
\text { sonian Inst. }\end{array}$ & Gonyleptoidea & Gonyleptidae & A. Ayala-Apaza, 2021 \\
\hline 110 & MUSM-ENT 0511946 & 1 & Hutamaia plei Tourinho \& Mendes, 2014* & Maynas & Río Napo & $\begin{array}{l}\text { Río Napo, terraza media } \\
\text { plana }\end{array}$ & 206 & $1^{\circ} 42^{\prime} 09.94 "$ & $74^{\circ} 47^{\prime} 09.03^{\prime \prime}$ & & $\mathrm{i}$ & 2009 & R. Yawarcani & Etiqueta & Consultoría EIA & Gonyleptoidea & Gonyleptidae & A. Ayala-Apaza, 2021 \\
\hline 111 & MUSM-ENT 0511976 & 1 & Hutamaia plei Tourinho \& Mendes, 2014* & Maynas & Aguas Negras & Camp. Catarindo & 199 & $0^{\circ} 39^{\prime} 34.1^{\prime \prime}$ & $75^{\circ} 15^{\prime} 38.4^{\prime \prime}$ & 17 & iii & 1994 & R. Ramirez & Etiqueta & $\begin{array}{l}\text { Exp. Bioamazonas- } \\
\text { MHN,UNMSM }\end{array}$ & Gonyleptoidea & Gonyleptidae & A. Ayala-Apaza, 2021 \\
\hline 112 & MUSM-ENT 0512120 & 6 & Hutamaia plei Tourinho \& Mendes, 2014* & Maynas & Río Nashiño & & 190 & $1923^{\prime 2} 25.7^{\prime \prime}$ & $75016^{\prime} 46.64^{\prime \prime}$ & 25 & xii & 2008 & R. Yawarcani & Etiqueta & Consultoría EIA & Gonyleptoidea & Gonyleptidae & A. Ayala-Apaza, 2021 \\
\hline 113 & MUSM-ENT 0512182 & 1 & Leiobuninae 1 & Maynas & Río Napo & & 194 & $1^{\circ} 51^{\prime} 42.5^{\prime \prime}$ & $75^{\circ} 24^{\prime} 06.1^{\prime \prime}$ & $23-24$ & ii & 2008 & W. Paredes & Etiqueta & Consultoría EIA & Gonyleptoidea & Agoristenidae & A. Ayala-Apaza, 2021 \\
\hline 114 & MUSM-ENT 0511986 & 1 & Manaosbiidae 1 & Maynas & Río Napo & & 210 & $1^{\circ} 45^{\prime} 23.44^{\prime \prime}$ & $74^{\circ} 43^{\prime} 22.93^{\prime \prime}$ & 11 & $\mathrm{i}$ & 2009 & W. Yawarcani & Etiqueta & Consultoría EIA & Gonyleptoidea & Manaosbiidae & A. Ayala-Apaza, 2021 \\
\hline 115 & MUSM-ENT 0511987 & 3 & Manaosbiidae 1 & Loreto & Río Samiria & Cocha Shinguito & 90 & $5^{\circ} 10^{\prime}$ & $74^{\circ} 39^{\prime}$ & 15 & v & 1990 & D. Silva & Etiqueta & $\begin{array}{l}\text { MHN-UNMSM/Smith- } \\
\text { sonian Inst. }\end{array}$ & Gonyleptoidea & Manaosbiidae & A. Ayala-Apaza, 2021 \\
\hline 116 & MUSM-ENT 0511996 & 1 & Manaosbiidae 1 & Maynas & Rio Napo & & 210 & $1^{\circ} 45^{\prime 23.44 "}$ & 7443'22.93" & 22 & xii & 2008 & W. Yawarcani & Etiqueta & Consultoría EIA & Gonyleptoidea & Manaosbiidae & A. Ayala-Apaza, 2021 \\
\hline 117 & MUSM-ENT 0511997 & 2 & Manaosbiidae 1 & Maynas & Rio Napo & & 190 & $0^{\circ} 58^{\prime} 48.80 "$ & $75^{\circ} 53^{2} 27.69 "$ & 11 & $\mathrm{i}$ & 2009 & R. Yawarcani & Etiqueta & Consultoría EIA & Gonyleptoidea & Manaosbiidae & A. Ayala-Apaza, 2021 \\
\hline 118 & MUSM-ENT 0512001 & 1 & Manaosbiidae 1 & Maynas & Río Napo & & 193 & $00^{\circ} 58^{\prime} 48.80^{\prime \prime}$ & $75^{\circ} 13^{\prime 2} 27.69^{\prime \prime}$ & 11 & $\mathrm{i}$ & 2009 & R. Yawarcani & Etiqueta & Consultoría EIA & Gonyleptoidea & Manaosbiidae & M. Medrano y A. Ayala, 2021 \\
\hline
\end{tabular}


Los LANiAToRes (ARACHNIDA: OPILIONES: GRASSATORES) DEL DEPARTAMENTO DE LORETO

\begin{tabular}{|c|c|c|c|c|c|c|c|c|c|c|c|c|c|c|c|c|c|}
\hline$\#$ & Código de ingreso & No. Ind & Especie/Morfoespecie & Provincia & Localidad & Lugar de colecta & Altitud & Latitud & Longitud & Dia & Mes & Año colectores & $\begin{array}{l}\text { Fuente para } \\
\text { georeferen- } \\
\text { ciación }\end{array}$ & Proyecto de colecta & Superfamilia & Familia & Identificación \\
\hline 119 & MUSM-ENT 0512090 & 3 & Manaosbiidae 1 & Maynas & Río Curaray & & 209 & $1{ }^{\circ} 56^{\prime} 5.7 "$ & $75^{\circ} 22^{\prime} 20.8^{\prime \prime}$ & 10 & ii & 2008 W. Paredes & Etiqueta & Consultoría EIA & Gonyleptoidea & Manaosbiidae & A. Ayala-Apaza, 2021 \\
\hline 120 & MUSM-ENT 0512186 & 4 & Manaosbiidae 1 & Maynas & Río Napo & & 194 & $1^{\circ} 51^{\prime} 42.5^{\prime \prime}$ & $75^{\circ} 24^{\prime} 06.1^{\prime \prime}$ & $23-24$ & ii & 2008 W. Paredes & Etiqueta & Consultoría EIA & Gonyleptoidea & Manaosbiidae & A. Ayala-Apaza, 2021 \\
\hline 121 & MUSM-ENT 0514085 & 1 & Manaosbiidae 1 & Maynas & Río Napo & Napo & 176 & $1^{\circ} 51^{\prime} 42.5 "$ & $75^{\circ} 24^{\prime} 06.1^{\prime \prime}$ & $24-25$ & ii & 2008 W. Paredes & Etiqueta & Consultoría EIA & Gonyleptoidea & Manaosbiidae & A. Ayala-Apaza, 2021 \\
\hline 122 & MUSM-ENT 0512141 & 1 & Meterginus aff. serratus Roewer, 1912 & Loreto & Río Samiria & Pithecia & 111 & $5^{\circ} 10^{\prime}$ & $74^{\circ} 39^{\prime}$ & 14 & viii & 1989 D. Silva & Etiqueta & $\begin{array}{l}\text { MHN-UNMSM/Smith- } \\
\text { sonian Inst. }\end{array}$ & ${ }^{h-}$ Gonyleptoidea & Cosmetidae & M. Medrano y A. Ayala, 2027 \\
\hline 123 & MUSM-ENT 0512361 & 1 & Meterginus serratus Roewer, 1912* & Maynas & Río Napo & & 210 & $1^{\circ} 45^{\prime 2} 23.44^{\prime \prime}$ & $34^{\circ} 43^{\prime} 22.93^{\prime \prime}$ & 22 & xii & 2008 W. Yawarcani & Etiqueta & Consultoría EIA & Gonyleptoidea & Cosmetidae & A. Ayala-Apaza, 2021 \\
\hline 124 & MUSM-ENT 0512377 & 1 & Meterginus serratus Roewer, 1912* & Requena & Jenaro Herrera & $\begin{array}{l}\text { Centro de Investigación } \\
\text { Jenaro Herrera }\end{array}$ & 500 & $04^{\circ} 55^{\prime}$ & $73^{\circ} 45^{\prime}$ & 25 & viii & 1988 D. Silva & Etiqueta & MHN-UNMSM & Gonyleptoidea & Cosmetidae & A. Ayala-Apaza, 2021 \\
\hline 125 & MUSM-ENT 0512449 & 1 & Meterginus serratus Roewer, 1912* & Requena & Jenaro Herrera & $\begin{array}{l}\text { Centro de Investigación } \\
\text { Jenaro Herrera }\end{array}$ & 500 & $04^{\circ} 55^{\prime}$ & $73^{\circ} 45^{\prime}$ & & viii & 1988 D. Silva & Etiqueta & MHN-UNMSM & Gonyleptoidea & Cosmetidae & M. Medrano y A. Ayala, 2027 \\
\hline 126 & MUSM-ENT 0512484 & 2 & Meterginus serratus Roewer, 1912* & Maynas & Río Napo & & 200 & $1^{\circ} 52^{2} 34.27 "$ & $74^{\circ} 47^{\prime} 33.27^{\prime \prime}$ & 1 & $\mathrm{i}$ & 2009 R, Yawarcani & Etiqueta & Consultoría EIA & Gonyleptoidea & Cosmetidae & A. Ayala-Apaza, 2021 \\
\hline 127 & MUSM-ENT 0512534 & 2 & Meterginus serratus Roewer, 1912* & Maynas & Río Nashiño & & 200 & $1^{\circ} 23^{\prime 2} 25.7^{\prime \prime}$ & $75^{\circ} 16^{\prime} 46.64 "$ & 25 & xii & 2008 R. Yawarcani & Etiqueta & Consultoría EIA & Gonyleptoidea & Cosmetidae & A. Ayala-Apaza, 2021 \\
\hline 128 & MUSM-ENT 0512540 & 1 & Meterginus serratus Roewer, $1912^{*}$ & Maynas & Río Napo & & 190 & $005848.80 "$ & $75^{\circ} 13^{\prime} 27.69 "$ & 11 & $i$ & 2009 R. Yawarcani & Etiqueta & Consultoría EIA & Gonyleptoidea & Cosmetidae & A. Ayala-Apaza, 2021 \\
\hline 129 & MUSM-ENT 0512593 & 1 & Meterginus serratus Roewer, 1912* & Requena & Jenaro Herrera & $\begin{array}{l}\text { Centro de Investigación } \\
\text { Jenaro Herrera }\end{array}$ & 500 & $04^{\circ} 55^{\prime}$ & $73^{\circ} 45^{\prime}$ & 24 & viii & 1988 D. Silva & Etiqueta & MHN-UNMSM & Gonyleptoidea & Cosmetidae & M. Medrano, 2021 \\
\hline 130 & MUSM-ENT 0514100 & 1 & Meterginus serratus Roewer, $1912^{*}$ & Maynas & Río Napo & & 190 & $0^{\circ} 58^{\prime} 48.80^{\prime \prime}$ & $75^{\circ} 13^{\prime 2} 27.69 "$ & 11 & $\mathrm{i}$ & 2009 R. Yawarcani & Etiqueta & Consultoría EIA & Gonyleptoidea & Cosmetidae & A. Ayala-Apaza, 2021 \\
\hline 131 & MUSM-ENT 0514473 & 6 & Meterginus serratus Roewer, 1912* & Maynas & Rio ltaya & Quebrada Yanayacu & 102 & $04^{\circ} 16^{\prime} 49.9^{\prime \prime}$ & $73^{\circ} 40^{\prime} 52.2^{\circ}$ & $12-20$ & vii & 2012 D. Silva & Etiqueta & MHN-UNMSM & Gonyleptoidea & Cosmetidae & A. Ayala-Apaza, 2021 \\
\hline 132 & MUSM-ENT 0514477 & 3 & Meterginus serratus Roewer, $1912^{*}$ & Maynas & Rio Itaya & Quebrada Yanayacu & 102 & $04^{\circ} 16^{\prime} 49.9^{\prime \prime}$ & $73^{\circ} 40^{\prime} 52.2^{\circ}$ & $12-20$ & vii & 2012 D. Silva & Etiqueta & MHN-UNMSM & Gonyleptoidea & Cosmetidae & A. Ayala-Apaza, 2021 \\
\hline 133 & MUSM-ENT 0512091 & 1 & obidosus 1 & Maynas & Río Curaray & & 209 & $1^{\circ} 56^{\prime} 5.7 "$ & $75^{\circ} 22^{\prime} 20.8^{\prime \prime}$ & 10 & ii & 2008 W. Paredes & Etiqueta & Consultoría EIA & Gonyleptoidea & Stygnidae & A. Ayala-Apaza, 2021 \\
\hline 134 & MUSM-ENT 0511842 & 2 & Obidosus albilineatus Roewer, 1957 & Maynas & Río Napo & $\begin{array}{l}\text { Rio Napo, terraza media } \\
\text { plana }\end{array}$ & 206 & $1^{\circ} 42^{\prime} 09.94 "$ & $74^{\circ} 47^{\prime} 09.03^{\prime \prime}$ & & $\mathrm{i}$ & 2009 R. Yawarcani & Etiqueta & Consultoría EIA & Gonyleptoidea & Stygnidae & A. Ayala-Apaza, 2021 \\
\hline 135 & MUSM-ENT 0511843 & 2 & Obidosus albilineatus Roewer, 1957 & Maynas & Río Napo & & 210 & ${ }^{\circ} 45^{2} 23.44^{\prime \prime}$ & $74^{\circ} 43^{\prime} 22.93^{\prime \prime}$ & 22 & xii & 2008 W. Yawarcani & Etiqueta & Consultoría EIA & Gonyleptoidea & Stygnidae & A. Ayala-Apaza, 2021 \\
\hline 136 & MUSM-ENT 0511845 & 1 & Obidosus albilineatus Roewer, 1957 & Maynas & Río Napo & & 210 & $1^{\circ} 95^{\prime 23.44^{\prime}}$ & $74^{\circ} 43^{\prime} 22.93^{\prime \prime}$ & 22 & xii & 2008 W. Yawarcani & Etiqueta & Consultoría EIA & Gonyleptoidea & Stygnidae & A. Ayala-Apaza, 2021 \\
\hline 137 & MUSM-ENT 0513733 & 2 & obidosus albilineatus Roewer, 1957 & Maynas & Rio Itaya & Quebrada Yanayacu & 102 & $04^{\circ} 16^{\prime} 49.99^{\prime \prime}$ & $73^{\circ} 40^{\prime} 52.2^{\prime \prime}$ & 25 & $x$ & 2018 D. Silva & Etiqueta & MHN-UNMSM & Gonyleptoidea & Stygnidae & A. Ayala-Apaza, 2021 \\
\hline 138 & MUSM-ENT 0514472 & 3 & Obidosus albilineatus Roewer, 1957 & Maynas & Rio Itaya & Quebrada Yanayacu & 102 & $04^{\circ} 16^{\prime} 49.9^{\prime \prime}$ & $73^{\circ} 40^{\prime} 52.2^{\prime \prime}$ & $12-20$ & vii & 2012 D. Silva & Etiqueta & MHN-UNMSM & Gonyleptoidea & Stygnidae & A. Ayala-Apaza, 2021 \\
\hline 139 & MUSM-ENT 0505855 & 1 & $\begin{array}{l}\text { Obidosus boibumba (Villarreal \& Pinto-da-Rocha, } \\
\text { 2006)* }\end{array}$ & Alto Amazonas & Yurimaguas & Iquitos & & $4^{\circ} 30^{\prime 2} 21.6^{\prime \prime}$ & $75^{\circ} 26^{\prime} 27.6^{\prime \prime}$ & 5 & v & $\begin{array}{l}2012 \text { C. Espinoza, C. } \\
\text { Ampudia }\end{array}$ & Google Earth & thiconsultoría EIA & Gonyleptoidea & Stygnidae & A. Ayala-Apaza, 2021 \\
\hline 140 & MUSM-ENT 0512560 & 1 & Paecilaema bilunatum (Wood, 1869) & Maynas & Aguas negras & Camp. Catarindo & 199 & $0^{\circ} 39^{\prime 34.1 " 1 "}$ & $75^{\circ} 15^{\prime} 38.4^{\prime \prime}$ & 18 & iii & 1994 R. Ramirez & Etiqueta & $\begin{array}{l}\text { Exp. Bioamazonas- } \\
\text { MHN, UNMSM }\end{array}$ & Gonyleptoidea & Cosmetidae & A. Ayala-Apaza, 2021 \\
\hline 141 & MUSM-ENT 0512589 & 1 & Paecilaema bilunatum (Wood, 1869) & Maynas & Aguas negras & Camp. Catarindo & 199 & $0^{\circ} 39^{\prime 34.1 " 1 "}$ & $75^{\circ} 15^{\prime} 38.4^{\prime \prime}$ & 17 & iii & 1994 R. Ramirez & Etiqueta & $\begin{array}{l}\text { Exp. Bioamazonas- } \\
\text { MHN,UNMSM }\end{array}$ & Gonyleptoidea & Cosmetidae & A. Ayala-Apaza, 2021 \\
\hline 142 & MUSM-ENT 0512363 & 1 & Paecilaemella multimaculata (Wood, 1869) & Maynas & Rio Napo & & 210 & $1^{\circ} 45^{2} 23.44^{\prime \prime}$ & $34^{\circ} 43^{\prime} 22.93^{\prime \prime}$ & 22 & xii & 2008 W. Yawarcani & Etiqueta & Consultoría EIA & Gonyleptoidea & Cosmetidae & A. Ayala-Apaza, 2021 \\
\hline 143 & MUSM-ENT 0514090 & 1 & Paecilaemella multimaculata (Wood, 1869) & Maynas & Río Curaray & Napo, colinas medias & 178 & $1^{\circ} 53^{\prime} 15.5^{\prime \prime}$ & $75^{\circ} 21^{\prime} 51.0^{\prime \prime}$ & 19 & ii & 2008 W. Paredes & Etiqueta & Consultoría EIA & Gonyleptoidea & Cosmetidae & A. Ayala-Apaza, 2021 \\
\hline 144 & MUSM-ENT 0514468 & 2 & Paecilaemella multimaculata (Wood, 1869) & Maynas & Río ltaya & Quebrada Yanayacu & 102 & $04^{\circ} 16^{\prime} 49.99^{\prime \prime}$ & $73^{\circ} 40^{\prime} 52.2^{\prime \prime}$ & $12-20$ & vii & 2012 D. Silva & Etiqueta & MHN-UNMSM & Gonyleptoidea & Cosmetidae & A. Ayala-Apaza, 2021 \\
\hline 145 & MUSM-ENT 0512363 & 1 & Paecilaemella multimaculata (Wood, 1869) & Maynas & Río Napo & & 210 & $1^{\circ} 45^{\prime} 23.44^{\prime \prime}$ & $34^{\circ} 43^{\prime} 22.93$ & 22 & xii & 2008 W. Yawarcani & Etiqueta & Consultoría EIA & Gonyleptoidea & Cosmetidae & A. Ayala-Apaza, 2021 \\
\hline 146 & MUSM-ENT 0512344 & 28 & Paecilaemula argentinoi Soares, 1970* & Requena & Jenaro Herrera & $\begin{array}{l}\text { Centro de Investigación } \\
\text { Jenaro Herrera }\end{array}$ & 500 & $04^{\circ} 55^{\prime}$ & $73^{\circ} 45^{\prime}$ & & viii & 1988 D. Silva & Etiqueta & MHN-UNMSM & Gonyleptoidea & Cosmetidae & A. Ayala-Apaza, 2021 \\
\hline 147 & MUSM-ENT 0512345 & 2 & Paecilaemula argentinoi Soares, 1970* & Requena & Jenaro Herrera & $\begin{array}{l}\text { Maniti, en palmeras } \\
\text { Astrocarium sp. }\end{array}$ & & $04^{\circ} 55^{\prime}$ & $73^{\circ} 45^{\prime}$ & 23 & $\mathrm{x}$ & 1988 J. Llosa & Google Earth & $\begin{array}{l}\text { ORSTOM/IIAP, ORS- } \\
\text { th:OM/MHN-UNMSM }\end{array}$ & Gonyleptoidea & Cosmetidae & A. Ayala-Apaza, 2021 \\
\hline 148 & MUSM-ENT 0512542 & 2 & Paecilaemula argentinoi Soares, $1970^{*}$ & Requena & Jenaro Herrera & $\begin{array}{l}\text { Centro de Investigación } \\
\text { Jenaro Herrera }\end{array}$ & & $04^{\circ} 55^{\prime}$ & $73^{\circ} 45^{\prime}$ & 1 & ix & 1990 P. Lozada & Google Earth & th:MHN-UNMSM & Gonyleptoidea & Cosmetidae & A. Ayala-Apaza, 2021 \\
\hline
\end{tabular}




\begin{tabular}{|c|c|c|c|c|c|c|c|c|c|c|c|c|c|c|c|c|c|c|}
\hline$\#$ & Código de ingreso & No. Ind. & Aspecie/Morfoespecie & Provincia & Localidad & Lugar de colecta & Altitud & Latitud & Longitud & Dia & Mes & Año & Colectores & $\begin{array}{l}\text { Fuente para } \\
\text { georeferen- } \\
\text { ciación }\end{array}$ & Proyecto de colecta & Superfamilia & Familia & Identificación \\
\hline 149 & MUSM-ENT 0512545 & 2 & Paecilaemula argentinoi Soares, $1970^{*}$ & Requena & Jenaro Herrera & $\mathrm{km} 4.5 \mathrm{JH}$-Pto Angamos & & $04^{\circ} 55^{\prime}$ & $73^{\circ} 45^{\prime}$ & 30 & v & 1990 & P. Lozada & Google Earth & ihMHN-UNMSM & Gonyleptoidea & Cosmetidae & A. Ayala-Apaza, 2021 \\
\hline 150 & MUSM-ENT 0512584 & 5 & Paecilaemula argentinoi Soares, 1970* & Requena & Jenaro Herrera & $\mathrm{km} 4.5 \mathrm{JH}$-Pto Angamos & & $04^{\circ} 55^{\prime}$ & $73^{\circ} 45^{\prime}$ & 31 & v & 1990 & P. Lozada & Etiqueta & MHN-UNMSM & Gonyleptoidea & Cosmetidae & M. Medrano, 2021 \\
\hline 151 & MUSM-ENT 0512590 & 1 & Paecilaemula argentinoi Soares, 1970* & Requena & Jenaro Herrera & $\begin{array}{l}\text { Purma } 2 \text { años. } \mathrm{km} 4.5 \\
\text { JH-Pto Angamos }\end{array}$ & & $04^{\circ} 55^{\prime}$ & $73^{\circ} 45^{\prime}$ & 27 & viii & 1990 & P. Lozada & Google Earth & inMHN-UNMSM & Gonyleptoidea & Cosmetidae & A. Ayala-Apaza, 2021 \\
\hline 152 & MUSM-ENT 0512591 & 2 & Paecilaemula argentino Soares, $1970^{*}$ & Requena & Jenaro Herrera & $\begin{array}{l}\text { Purma } 2 \text { años. } \mathrm{km} 4.5 \\
\text { JH-Pto Angamos }\end{array}$ & & $04^{\circ} 55^{\prime}$ & $73^{\circ} 45^{\prime}$ & 30 & viii & 1990 & P. Lozada & Google Earth & inMHN-UNMSM & Gonyleptoidea & Cosmetidae & M. Medrano, 2021 \\
\hline 153 & MUSM-ENT 0514172 & 1 & Paecilaemula argentino Soares, $1970^{*}$ & Requena & Jenaro Herrera & $\begin{array}{l}\text { Centro de Investigación } \\
\text { Jenaro Herrera }\end{array}$ & 500 & $04^{\circ} 55^{\prime}$ & $73^{\circ} 45^{\prime}$ & & viii & 1988 & D. Silva & Etiqueta & MHN-UNMSM & Gonyleptoidea & Cosmetidae & $\begin{array}{l}\text { O. Villarreal-Manzanilla y A. } \\
\text { Ayala-Apaza, } 2021 \text {. }\end{array}$ \\
\hline 154 & MUSM-ENT 0514493 & 3 & Phareicranaus 1 & Maynas & Río Itaya & Quebrada Yanayacu & 102 & $04^{\circ} 16^{\prime} 49.9^{\prime \prime}$ & $73^{\circ} 40^{\prime} 52.2^{\prime \prime}$ & 25 & $x$ & 2018 & D. Silva & Etiqueta & MHN-UNMSM & Gonyleptoidea & Cranaidae & $\begin{array}{l}\text { O. Villarreal-Manzanilla y A. } \\
\text { Ayala-Apaza, } 2021 \text {. }\end{array}$ \\
\hline 155 & MUSM-ENT 0507355 & 1 & Phareicranaus 2 & Alto Amazonas & Yurimaguas & quitos. Miraflores & 69 & $4^{\circ} 30^{\prime}$ & $75^{\circ} 26^{\prime}$ & 10 & v & 2012 & & Etiqueta & Consultoría EIA & Gonyleptoidea & Cranaidae & $\begin{array}{l}\text { O. Villarreal-Manzanilla y A. } \\
\text { Ayala-Apaza, } 2021\end{array}$ \\
\hline 156 & MUSM-ENT 0511969 & 1 & Phareicranaus 2 & Maynas & Río Napo & & 210 & $1^{\circ} 45^{2} 23.44^{\prime \prime}$ & $74^{\circ} 43^{\prime} 22.93^{\prime \prime}$ & 22 & xii & 2008 & W. Yawarcani & Etiqueta & Consultoría EIA & Gonyleptoidea & Cranaidae & $\begin{array}{l}\text { O. Villarreal-Manzanilla y A. } \\
\text { Avall-Apaza, } 2021\end{array}$ \\
\hline 157 & MUSM-ENT 0511973 & 1 & Phareicranaus 2 & Maynas & Río Curaray & & & $1^{\circ} 47^{\prime} 59.6^{\prime}$ & $75^{\circ} 28^{\prime} 18.0^{\prime \prime}$ & 8 & ii & 2008 & W. Yawarcani & Etiqueta & Consultoría EIA & Gonyleptoidea & Cranaidae & $\begin{array}{l}\text { O. Villarreal-Manzanilla y A. } \\
\text { Ayala-Apaza, } 2021\end{array}$ \\
\hline 158 & MUSM-ENT 0512087 & 2 & Phareicranaus 2 & Maynas & Río Curaray & & 209 & $1{ }^{\circ} 56^{\prime} 5.7 "$ & $75^{\circ} 22^{\prime} 20.8^{\prime \prime}$ & 10 & ii & 2008 & W. Paredes & Etiqueta & Consultoría EIA & Gonyleptoidea & Cranaidae & $\begin{array}{l}\text { O. Villarreal-Manzanilla y A. } \\
\text { Avall-Apaza, } 2021 \text {. }\end{array}$ \\
\hline 159 & MUSM-ENT 0514116 & 1 & Phareicranaus 3 & Maynas & Río Curaray & & 162 & $1^{\circ} 31^{\prime 4} 46.2 "$ & $75^{\circ} 25^{\prime} 30.4^{\prime \prime}$ & 10 & ii & 2000 & W. Paredes & Etiqueta & Consultoría EIA & Gonyleptoidea & Cranaidae & $\begin{array}{l}\text { O. Villarreal-Manzanilla y A. } \\
\text { Ayala-Apaza, } 2021 \text {. }\end{array}$ \\
\hline 160 & MUSM-ENT 0514470 & 7 & Phareicranaus aff. calcarifer (simon, 1879) & Maynas & Rio ltaya & Quebrada Yanayacu & 102 & $04^{\circ} 16^{\prime} 49.9^{\prime \prime}$ & $73^{\circ} 40^{\prime} 52.2^{\prime \prime}$ & $\mid 12-20$ & vii & 2012 & D. Silva & Etiqueta & MHN-UNMSM & Gonyleptoidea & Cranaidae & $\begin{array}{l}\text { O. Villarreal-Manzanilla y A. } \\
\text { Avala-Apaza, 2021 }\end{array}$ \\
\hline 161 & MUSM-ENT 0514469 & 1 & Phareicranaus cf. manauara (Pinto-da-Rocha, 1994) & Maynas & Rio Itaya & Quebrada Yanayacu & 102 & $04^{\circ} 16^{\prime} 49.9^{\prime \prime}$ & $73^{\circ} 40^{\prime} 52.2^{\prime \prime}$ & 25 & $x$ & 2018 & D. Silva & Etiqueta & MHN-UNMSM & Gonyleptoidea & Cranaidae & A. Ayala-Apaza, 2021 \\
\hline 162 & MUSM-ENT 0501283 & 1 & Phareicranaus singularis (H. Soares, 1970) & Maynas & Río Napo & & 200 & ${ }^{\circ} 51^{1} 13.16 "$ & $74^{\circ} 47^{\prime} 26.38^{\prime \prime}$ & 13 & $\mathrm{i}$ & 2009 & W. Yawarcani & Etiqueta & Consultoría EIA & Gonyleptoidea & Cranaidae & A. Ayala-Apaza, 2021 \\
\hline 163 & MUSM-ENT 0511981 & 1 & Phareicranaus singularis (H. Soares, 1970) & Maynas & Río Napo & & & $0^{\circ} 58^{\prime} 48.81^{\prime \prime}$ & $75^{\circ} 13^{\prime 27.69 "}$ & 11 & $\mathrm{i}$ & 2009 & R. Yawarcani & Etiqueta & Consultoría EIA & Gonyleptoidea & Cranaidae & $\begin{array}{l}\text { A. Ayala-Apaza y A. Pérez-Gonzá } \\
\text { lez, } 2020 \text {. }\end{array}$ \\
\hline 164 & MUSM-ENT 0511802 & 4 & Samoidae 1 & Loreto & Río Samiria & Cocha Shinguito & 90 & $5^{\circ} 10^{\prime}$ & $74^{\circ} 39^{\prime}$ & 16 & $\mathrm{v}$ & 1990 & T. Erwin & Etiqueta & $\begin{array}{l}\text { MHN-UNMSM/Smith } \\
\text { sonian Inst. }\end{array}$ & hamooidea & Samoidae & $\begin{array}{l}\text { A. Pérez-González y A. Ayala- } \\
\text { Apaza, } 2020\end{array}$ \\
\hline 165 & MUSM-ENT 0511993 & 3 & Samoidae 1 & Loreto & Río Samiria & Cocha Shinguito & 90 & $5^{\circ} 10^{\prime}$ & $7^{70^{\circ} 39^{\prime}}$ & 15 & v & 1990 & T. Erwin & Etiqueta & $\begin{array}{l}\text { MHN-UNMSM/Smith } \\
\text { sonian Inst. }\end{array}$ & -Samooidea & Samoidae & $\begin{array}{l}\text { A. Ayala-Apaza y A. Pérez-Gonzá } \\
\text { lez, 2020 }\end{array}$ \\
\hline 166 & MUSM-ENT 0512182 & 1 & Samoidae 1 & Loreto & Rio Samiria & Cocha Shinguito & 90 & $5^{\circ} 10^{\prime}$ & $74^{\circ} 39^{\prime}$ & & v-vi & 1990 & T. Erwin & Etiqueta & $\begin{array}{l}\text { MHN-UNMSM/Smith } \\
\text { sonian Inst. }\end{array}$ & hamooidea & Samoidae & $\begin{array}{l}\text { A. Pérez-González y A. Ayala- } \\
\text { Apaza, } 2020\end{array}$ \\
\hline 167 & MUSM-ENT 0512241 & 1 & Samoidae 1 & Loreto & Río Samiria & Cocha Shinguito & 90 & $5^{\circ} 10^{\prime}$ & $74^{\circ} 39^{\prime}$ & 12 & v & 1990 & T. Erwin & Etiqueta & $\begin{array}{l}\text { MHN-UNMSM/Smith } \\
\text { sonian Inst. }\end{array}$ & h-Samooidea & Samoidae & $\begin{array}{l}\text { A. Ayala-Apaza y A. Pérez-Gonzá } \\
\text { lez, } 2020\end{array}$ \\
\hline 168 & MUSM-ENT 0512496 & 9 & Samoidae 1 & Loreto & Rio Samiria & Cocha Shinguito & 90 & $5^{\circ} 10^{\prime}$ & $74^{\circ} 39^{\prime}$ & 30 & v & 1990 & T. Erwin & Etiqueta & $\begin{array}{l}\text { MHN-UNMSM/Smith } \\
\text { sonian Inst. }\end{array}$ & h- Samooidea & Samoidae & $\begin{array}{l}\text { A. Pérez-González y A. Ayala- } \\
\text { Apaza, } 2020\end{array}$ \\
\hline 169 & MUSM-ENT 0512556 & 4 & Samoidae 1 & Loreto & Rio Samiria & Cocha Shinguito & 90 & $5^{\circ} 10^{\prime}$ & $74^{\circ} 39^{\prime}$ & & v & 1990 & D. Silva & Etiqueta & $\begin{array}{l}\text { MHN-UNMSM/Smith } \\
\text { sonian Inst. }\end{array}$ & h- Samooidea & Samoidae & $\begin{array}{l}\text { A. Ayala-Apaza y A. Pérez-Gonzá } \\
\text { lez, } 2020\end{array}$ \\
\hline 170 & MUSM-ENT 0512559 & 2 & Samoidae 1 & Loreto & Río Samiria & Cocha Shinguito & 90 & $5^{\circ} 10^{\prime}$ & $74^{\circ} 39^{\prime}$ & 20 & v & 1990 & & Etiqueta & $\begin{array}{l}\text { MHN-UNMSM/Smith } \\
\text { sonian Inst. }\end{array}$ & h-samooidea & Samoidae & $\begin{array}{l}\text { A. Pérez-González y A. Ayala- } \\
\text { Apaza, } 2020\end{array}$ \\
\hline 171 & MUSM-ENT 0514170 & 3 & Samoidae 1 & Loreto & Río Samiria & Cocha Shinguito & 90 & $5^{\circ} 10^{\prime}$ & $74^{\circ} 39^{\prime}$ & 16 & v & 1990 & T. Erwin & Etiqueta & $\begin{array}{l}\text { MHN-UNMSM/Smith } \\
\text { sonian Inst. }\end{array}$ & h-samooidea & Samoidae & $\begin{array}{l}\text { A. Ayyla-Apaza y A. Pérez-Gonzá } \\
\text { lez, 2020 }\end{array}$ \\
\hline 172 & MUSM-ENT 0514174 & 4 & Samoidae 1 & Loreto & Río Samiria & Cocha Shinguito & 90 & $5^{\circ} 10^{\prime}$ & $74^{\circ} 39^{\prime}$ & 23 & v & 1990 & T. Erwin & Etiqueta & NLRP/Carabidae & Samooidea & Samoidae & $\begin{array}{l}\text { A. Pérez-González y A. Ayala- } \\
\text { Apaza, } 2020\end{array}$ \\
\hline 173 & MUSM-ENT 0514176 & 2 & Samoidae 1 & Loreto & Río Samiria & Cocha Shinguito & 90 & $5^{\circ} 10^{\prime}$ & $74^{\circ} 39^{\prime}$ & 22 & v & 1990 & T. Erwin & Etiqueta & NLRP/Carabidae & Samooidea & Samoidae & A. Ayala-Apaza, 2020 \\
\hline 174 & MUSM-ENT 0514504 & 1 & Samooidea 1 & Maynas & Río Napo & Napo & 176 & $1^{\circ} 51^{4} 42.5^{\prime \prime}$ & $75^{\circ} 24^{\prime} 06.1^{\prime \prime}$ & $24-25$ & ii & 2008 & W. Paredes & Etiqueta & Consultoría EIA & Samooidea & & A. Ayala-Apaza, 2021 \\
\hline 175 & MUSM-ENT 0512531 & 1 & Sibambea rotunda Roewer, $1917^{*}$ & Maynas & Río Napo & Terraza media plana & 206 & $1^{\circ} 42^{\prime} 09.94 "$ & $74^{\circ} 47^{\prime} 09.03^{\prime \prime}$ & & $\mathrm{i}$ & 2009 & R. Yawarcani & Etiqueta & Consultoría EIA & Gonyleptoidea & Cosmetidae & A. Ayala-Apaza, 2021 \\
\hline 176 & MUSM-ENT 0512533 & 1 & Sibambea rotunda Roewer, 1917* & Maynas & Rio Nashiño & & 200 & $1^{\circ 22325.7 " 1 "}$ & $75^{\circ} 16^{\prime} 46.64^{\prime \prime}$ & 25 & xii & 2008 & R. Yawarcani & Etiqueta & Consultoría EIA & Gonyleptoidea & Cosmetidae & A. Ayala-Apaza, 2021 \\
\hline 177 & MUSM-ENT 0514096 & 1 & Sibambea rotunda Roewer, $1917^{*}$ & Maynas & Rio Curaray & & 209 & $1^{1} 56^{\prime} 5.7 "$ & $75^{\circ} 22^{\prime} 20.8^{\prime \prime}$ & 10 & ii & 2008 & W. Paredes & Etiqueta & Consultoría EIA & Gonyleptoidea & Cosmetidae & A. Ayala-Apaza, 2021 \\
\hline 178 & MUSM-ENT 0500810 & 1 & Stenostygnus pusio Simon, 1879* & Maynas & quitos & R.N. Allpahuayo-Mishana, & & $4^{\circ} 30^{\prime} 14.4^{\prime \prime}$ & $73^{\circ} 34^{\prime} 44.4^{\prime \prime}$ & & v & 2005 & A. Catenazzi & Google Earth & hMHN-UNMSM/IIAP & Samooidea & Biantidae & $\begin{array}{l}\text { A. Pérez-González y A. Ayala- } \\
\text { Apaza, } 2020\end{array}$ \\
\hline
\end{tabular}




\begin{tabular}{|c|c|c|c|c|c|c|c|c|c|c|c|c|c|c|c|c|c|c|}
\hline$\#$ & Código de ingreso & No. In & 1. Especie/Morfoespecie & Provincia & Localidad & Lugar de colecta & $\begin{array}{c}\text { Altitud } \\
\mathrm{m}\end{array}$ & Latitud & Longitud & Dia & Mes & Año & Colectores & $\begin{array}{l}\text { Fuente para } \\
\text { georeferen- } \\
\text { ciación }\end{array}$ & Proyecto de colecta & Superfamilia & Familia & Identificación \\
\hline 179 & MUSM-ENT 0511812 & 1 & Stenostyynus pusio Simon, 1879* & Loreto & Río Samiria & Cocha Shinguito & 90 & $5^{\circ} 10^{\prime}$ & $74^{\circ} 39^{\prime}$ & & $i v-v$ & 1990 & T. Erwin & Etiqueta & $\begin{array}{l}\text { MHN-UNMSM/Smith- } \\
\text { sonian Inst. }\end{array}$ & Samooidea & Biantidae & $\begin{array}{l}\text { A. Pérez-González y A. Ayala- } \\
\text { Apaza, 2020 }\end{array}$ \\
\hline 180 & MUSM-ENT 0511810 & 1 & Stygnomma 1 & Loreto & Río Samiria & Cocha Shinguito & 90 & $5^{\circ} 10^{\prime}$ & $74^{\circ} 39^{\prime}$ & & $i v-v$ & 1990 & T. Erwin & Etiqueta & $\begin{array}{l}\text { MHN-UNMSM/Smith- } \\
\text { sonian Inst. }\end{array}$ & Samooidea & Stygnommatidae & A. Ayala-Apaza, 2021 \\
\hline 181 & MUSM-ENT 0511811 & 2 & Stygnomma 1 & Maynas & Aguas Negras & Camp. Catarindo & 199 & $0^{\circ} 39^{\prime 344.1 "}$ & $75^{\circ} 15^{\prime} 38.4^{\prime \prime}$ & 17 & iii & 1994 & R. Ramírez & Etiqueta & $\begin{array}{l}\text { Exp. Bioamazonas- } \\
\text { MHN,UNMSM }\end{array}$ & Samooidea & Stygnommatidae & A. Ayala-Apaza, 2021 \\
\hline 182 & MUSM-ENT 0514513 & 1 & Stygnomma 1 & Loreto & Río Samiria & Cocha Shinguito & 90 & $5^{\circ} 10^{\prime}$ & $74^{\circ} 39^{\prime}$ & & $i v-v$ & 1990 & T. Erwin & Etiqueta & $\begin{array}{l}\text { MHN-UNMSM/Smith- } \\
\text { sonian Inst. }\end{array}$ & Samooidea & Stygnommatidae & A. Ayala-Apaza, 2021 \\
\hline 183 & MUSM-ENT 0512200 & 1 & Stygnus 1 & Loreto & Río Samiria & Cocha Shinguito & 90 & $5^{\circ} 10^{\prime}$ & $74^{\circ} 39^{\prime}$ & 26 & $\mathrm{v}$ & 1990 & T. Erwin & Etiqueta & $\begin{array}{l}\text { MHN-UNMSM/Smith- } \\
\text { sonian Inst. }\end{array}$ & Gonyleptoidea & Stygnidae & A. Ayala-Apaza, 2021 \\
\hline 184 & MUSM-ENT 0512497 & 1 & Stygnus 1 & Loreto & Río Samiria & Cocha Shinguito & 90 & $5^{\circ} 10^{\prime}$ & $74^{\circ} 39^{\prime}$ & 30 & $\mathrm{v}$ & 1990 & T. Erwin & Etiqueta & $\begin{array}{l}\text { MHN-UNMSM/Smith- } \\
\text { sonian Inst. }\end{array}$ & Gonyleptoidea & Stygnidae & A. Ayala-Apaza, 2021 \\
\hline 185 & MUSM-ENT 0505273 & 1 & Stygnus klugi (Goodnight \& Goodnight, 1943) & Maynas & Rio ltaya & Quebrada Yanayacu & 102 & $04^{\circ} 16^{\prime} 49.9^{\prime \prime}$ & $73^{\circ} 40^{\circ} 52.2^{\prime \prime}$ & 19 & vii & 2012 & D. Silva & Etiqueta & Consultoría EIA & Gonyleptoidea & Stygnidae & R. Pinto-da-Rocha, 1993 \\
\hline 186 & MUSM-ENT 0507193 & 1 & Stygnus klugi (Goodnight \& Goodnight, 1943) & Alto Amazonas & Yurimaguas & Iquitos & & $4^{\circ} 30^{\prime} 21.6 "$ & $75^{\circ} 26^{\prime} 27.6^{\prime \prime}$ & 5 & $v$ & 2012 & C. Espinoza, C. & Google Earthi & hiconsultoría EIA & Gonyleptoidea & Stygnidae & A. Ayala, 2021 \\
\hline 187 & MUSM-ENT 0507500 & 1 & Stygnus klugi (Goodnight \& Goodnight, 1943) & Requena & Jenaro Herrera & $\begin{array}{l}\text { Centro de Investigación } \\
\text { Jenaro Herrera }\end{array}$ & 100 & $04^{\circ} 55^{\prime}$ & $73^{\circ} 45^{\prime}$ & 24 & viii & 1988 & D. Silva & Etiqueta & MHN-UNMSM & Gonyleptoidea & Stygnidae & R. Pinto-da-Rocha, 1993 \\
\hline 188 & MUSM-ENT 0511829 & 1 & Stygnus klugi (Goodnight \& Goodnight, 1943) & Ucayali & Bajo Inahuaillo & Campamento Venado & 300 & $06^{\circ} 51$ & $75^{\circ} 06^{\prime}$ & 14 & vii & 1988 & R. Fernandez & Etiqueta & MHN-UNMSM & Gonyleptoidea & Stygnidae & R. Pinto-da-Rocha, 1993 \\
\hline 189 & MUSM-ENT 0511834 & 1 & Stygnus klugi (Goodnight \& Goodnight, 1943) & Requena & Jenaro Herrera & $\begin{array}{l}\text { Centro de investigación } \\
\text { Jenaro Herrera, en pal- } \\
\text { meras Astrocarium sp. }\end{array}$ & 100 & $04^{\circ} 55^{\prime}$ & $73^{\circ} 45^{\prime}$ & 23 & viii & 1988 & B. Dilva & Etiqueta & MHN-UNMSM & Gonyleptoidea & Stygnidae & R. Pinto-da-Rocha, 1993 \\
\hline 190 & MUSM-ENT 0511835 & 1 & Stygnus Klugi (Goodnight \& Goodnight, 1943) & Requena & Jenaro Herrera & $\begin{array}{l}\text { Centro de investigación } \\
\text { Jenaro Herrera, en pal- } \\
\text { meras Astrocarium sp. }\end{array}$ & 100 & $04^{\circ} 55^{\prime}$ & $73^{\circ} 45^{\prime}$ & 23 & viii & 1988 & D. Silva & Etiqueta & MHN-UNMSM & Gonyleptoidea & Stygnidae & A. Ayala-Apaza, 2021 \\
\hline 191 & MUSM-ENT 0511837 & 1 & Stygnus Klugi (Goodnight \& Goodnight, 1943) & Loreto & Río Samiria & $\begin{array}{l}\text { Puesto de vigilancia Boca } \\
\text { del Samiria, bosque }\end{array}$ & & $4^{\circ} 41^{\prime}$ & $74^{\circ} 20^{\prime}$ & 11 & viii & 1989 & D. Silva & Etiqueta & $\begin{array}{l}\text { MHN-UNMSM/Smith- } \\
\text { sonian Inst. }\end{array}$ & Gonyleptoidea & Stygnidae & A. Ayala-Apaza, 2021 \\
\hline 192 & MUSM-ENT 0511854 & 1 & Stygnus Klugi (Goodnight \& Goodnight, 1943) & Loreto & Río Samiria & Cocha Shinguito & 90 & $5^{\circ} 10^{\prime}$ & $74^{\circ} 39^{\prime}$ & 17 & $\mathrm{v}$ & 1990 & T. Erwin & Etiqueta & $\begin{array}{l}\text { MHN-UNMSM/Smith- } \\
\text { sonian Inst. }\end{array}$ & Gonyleptoidea & Stygnidae & A. Ayala-Apaza, 2021 \\
\hline 193 & MUSM-ENT 0511855 & 1 & Stygnus Klugi (Goodnight \& Goodnight, 1943) & Loreto & Río Samiria & $\begin{array}{l}\text { Cocha Shinguito, en } \\
\text { bromeliacea }\end{array}$ & 90 & $5^{\circ} 10^{\prime}$ & $74^{\circ} 39^{\prime}$ & 7 & $\mathrm{v}$ & 1990 & T. Erwin & Etiqueta & $\begin{array}{l}\text { MHN-UNMSM/Smith- } \\
\text { sonian Inst. }\end{array}$ & Gonyleptoidea & Stygnidae & A. Ayala-Apaza, 2021 \\
\hline 194 & MUSM-ENT 0511859 & 1 & Stygnus Klugi (Goodnight \& Goodnight, 1943) & Loreto & Rí Pacayacu & $\begin{array}{l}2 \mathrm{~km} \text { from Comunidad } \\
\text { San Miguel }\end{array}$ & 134 & $04^{\circ} 05^{\prime} 59.2^{\prime \prime}$ & $75^{\circ 22} 37.6^{\prime \prime}$ & 30 & iii & 2008 & W. Paredes & Etiqueta & Consultoría EIA & Gonyleptoidea & Stygnidae & A. Ayala-Apaza, 2021 \\
\hline 195 & MUSM-ENT 0512034 & 4 & Stygnus klugi (Goodnight \& Goodnight, 1943) & Maynas & Río Napo & Rio Napo, colinas medias & 176 & ${ }^{\circ} 51^{\prime} 42.5^{\prime \prime}$ & $75^{\circ} 24^{\prime} 06.1 "$ & $24-26$ & ii & 2008 & W. Paredes & Etiqueta & Consultoría EIA & Gonyleptoidea & Stygnidae & A. Ayala-Apaza, 2021 \\
\hline 196 & MUSM-ENT 0512093 & 1 & Stygnus klugi (Goodnight \& Goodnight, 1943) & Maynas & Río Curaray & & 162 & $1^{\circ} 31^{\prime} 46.2^{\prime \prime}$ & $75^{\circ} 25^{\prime} 30.4^{\prime \prime}$ & $28-29$ & ii & 2008 & W. Paredes & Etiqueta & Consultoría EIA & Gonyleptoidea & Stygnidae & A. Ayala-Apaza, 2021 \\
\hline 197 & MUSM-ENT 0512498 & 1 & Stygnus klugi (Goodnight \& Goodnight, 1943) & Loreto & Río Samiria & Cocha Shinguito & 90 & $5^{\circ} 10^{\prime}$ & $74^{\circ} 39^{\prime}$ & 30 & $v$ & 1990 & T. Erwin & Etiqueta & $\begin{array}{l}\text { MHN-UNMSM/Smith- } \\
\text { sonian Inst. }\end{array}$ & Gonyleptoidea & Stygnidae & A. Ayala-Apaza, 2021 \\
\hline 198 & MUSM-ENT 0514110 & 1 & Stygnus klugi (Goodnight \& Goodnight, 1943) & Requena & Jenaro Herrera & $\begin{array}{l}\text { Maniti, en palmeras } \\
\text { Astrocarium sp. }\end{array}$ & & $04^{\circ} 55^{\prime}$ & $73^{\circ} 45^{\prime}$ & 23 & $\mathrm{x}$ & 1988 & 3 I. Llosa & Google Earth & $\begin{array}{l}\text { ORSTOM/IIAP, ORS- } \\
\text { TOM/MHN-UNMSM }\end{array}$ & Gonyleptoidea & Stygnidae & A. Ayala-Apaza, 2021 \\
\hline 199 & MUSM-ENT 0511839 & 1 & Stygnus 1 & Maynas & Rio Nashiño & & 190 & $1^{\circ} 23 ' 39.36^{\prime \prime}$ & $75^{\circ} 13^{\prime} 51.86^{\prime \prime}$ & 23 & xii & 2008 & R. Yawarcani & Etiqueta & Consultoría EIA & Gonyleptoidea & Stygnidae & A. Ayala-Apaza, 2021 \\
\hline 200 & MUSM-ENT 0511840 & 2 & Stygnus 1 & Maynas & Río Napo & & 193 & $0^{\circ} 58^{\prime} 48.80^{\prime \prime}$ & $75^{\circ} 13^{\prime 2} 27.69^{\prime \prime}$ & 11 & i & 2009 & R. Yawarcani & Etiqueta & Consultoría EIA & Gonyleptoidea & Stygnidae & A. Ayala-Apaza, 2021 \\
\hline 201 & MUSM-ENT 0512434 & 1 & Taito aff. insperatus Kury \& Barros, 2014 & Maynas & Río Curaray & & & $1^{\circ} 35^{\prime 16.1 "}$ & $75^{\circ} 24^{\prime} 19.77^{\prime \prime}$ & 15 & ii & 2008 & W. Yawarcani & Etiqueta & Consultoría EIA & Gonyleptoidea & Cosmetidae & A. Ayala-Apaza, 2021 \\
\hline 202 & MUSM-ENT 0512530 & 4 & Taito aff. insperatus Kury \& Barros, 2014 & Maynas & Río Napo & Terraza media plana & 206 & $1^{\circ} 42^{\prime} 09.94^{\prime \prime}$ & $74^{\circ} 47^{\prime} 09.03^{\prime \prime}$ & & $i$ & 2009 & R. Yawarcani & Etiqueta & Consultoría EIA & Gonyleptoidea & Cosmetidae & A. Ayala-Apaza, 2021 \\
\hline 203 & MUSM-ENT 0512180 & 2 & Taito insperatus Kury \& Barros, 2014* & Maynas & Río Curaray & & 216 & ${ }^{\circ} 35^{\prime} 16.1 "$ & $75^{\circ} 24^{\prime} 19.7^{\prime \prime}$ & 29 & ii & 2008 & W. Paredes & Etiqueta & Consultoría EIA & Gonyleptoidea & Cosmetidae & A. Ayala-Apaza, 2021 \\
\hline 204 & MUSM-ENT 0512481 & 1 & Taito insperatus Kury \& Barros, 2014* & Maynas & Río Napo & & 200 & $1^{\circ} 16^{\prime} 40.62 "$ & $7^{\circ} 17^{\prime} 18.96^{\prime \prime}$ & 28 & xii & 2008 & R. Yawarcani & Etiqueta & Consultoría EIA & Gonyleptoidea & Cosmetidae & A. Ayala-Apaza, 2021 \\
\hline 205 & MUSM-ENT 0512539 & 1 & Taito insperatus Kury \& Barros, 2014* & Maynas & Rio Napo & & 190 & $1^{\circ} 12^{\prime} 33.8^{\prime \prime}$ & $75^{\circ} 22^{\prime} 32^{\prime \prime}$ & 2 & $\mathrm{i}$ & 2009 & R. Yawarcani & Etiqueta & Consultoría EIA & Gonyleptoidea & Cosmetidae & A. Ayala-Apaza, 2021 \\
\hline 206 & MUSM-ENT 0514106 & 3 & Taito insperatus Kury \& Barros, 2014* & Maynas & Río Napo & & 190 & $0^{\circ} 58^{\prime} 48.80 "$ & $75^{\circ} 13^{\prime} 27.69 "$ & 11 & $i$ & 2009 & R. Yawarcani & Etiqueta & Consultoría EIA & Gonyleptoidea & Cosmetidae & A. Ayala-Apaza, 2021 \\
\hline 207 & MUSM-ENT 0514114 & 1 & Taito insperatus Kury \& Barros, 2014* & Maynas & Río Curaray & & 162 & ${ }^{\circ} 31^{\prime} 46.2^{\prime \prime}$ & $75^{\circ} 25^{\prime} 30.4^{\prime \prime}$ & & & & W. Paredes & Etiqueta & Consultoría EIA & Gonyleptoidea & Cosmetidae & $\begin{array}{l}\text { A. Ayala-Apaza y C. Pinzón-M., } \\
2021\end{array}$ \\
\hline 208 & MUSM-ENT 0512437 & 1 & Taito rorschachi Kury \& Barros, 2014* & Loreto & Río Samiria & Cocha Shinguito & 90 & $5^{\circ} 10^{\prime}$ & $74^{\circ} 39^{\prime}$ & & $i v-v$ & 1990 & T. Erwin & Etiqueta & $\begin{array}{l}\text { MHN-UNMSM/Smith- } \\
\text { sonian Inst. }\end{array}$ & Gonyleptoidea & Cosmetidae & A. Ayala-Apaza, 2021 \\
\hline
\end{tabular}




\begin{tabular}{|c|c|c|c|c|c|c|c|c|c|c|c|c|c|c|c|c|c|c|}
\hline \# & Código de ingreso & No. Inc & 1.Especie/Morfoespecie & Provincia & Localidad & Lugar de colecta & Altitud & d Latitud & Longitud & Dia & Mes & Año & Colectores & $\begin{array}{l}\text { Fuente para } \\
\text { georeferen- } \\
\text { ciación }\end{array}$ & Proyecto de colecta & Superfamilia & Familia & Identificación \\
\hline 209 & MUSM-ENT 0512463 & 1 & Taito rorschachi Kury \& Barros, 2014* & Requena & Jenaro Herrera & $\begin{array}{l}\text { Maniti, en palmeras } \\
\text { Astrocarium sp. }\end{array}$ & & $04^{\circ} 55^{\prime}$ & $73^{\circ} 45^{\prime}$ & 23 & $x$ & 1988 & I. Llosa & Google Earth & $\begin{array}{l}\text { OORSTOM/IIAP, ORS- } \\
\text { TOM/MHN-UNMSM }\end{array}$ & Gonyleptoidea & Cosmetidae & A. Ayala-Apaza, 2021 \\
\hline 210 & MUSM-ENT 0512544 & 6 & Taito rorschachi Kury \& Barros, 2014* & Loreto & Rio Samiria & Cocha Shinguito & 90 & $5^{\circ} 10^{\prime}$ & $74^{\circ} 39^{\prime}$ & 15 & $\mathrm{v}$ & 1990 & & Etiqueta & $\begin{array}{l}\text { MHN-UNMSM/Smith- } \\
\text { sonian Inst. }\end{array}$ & Gonyleptoidea & Cosmetidae & A. Ayala-Apaza, 2021 \\
\hline 211 & MUSM-ENT 0512554 & 14 & Taito rorschachi Kury \& Barros, 2014* & Loreto & Rio Samiria & Cocha Shinguito & 90 & $5^{\circ} 10^{\prime}$ & $74^{\circ} 39^{\prime}$ & 15 & v & 1990 & D. Silva & Etiqueta & $\begin{array}{l}\text { MHN-UNMSM/Smith- } \\
\text { sonian Inst. }\end{array}$ & Gonyleptoidea & Cosmetidae & A. Ayala-Apaza, 2021 \\
\hline 212 & MUSM-ENT 0512555 & 9 & Taito rorschachi Kury \& Barros, 2014* & Loreto & Rio Samiria & Cocha Shinguito & 90 & $5^{\circ} 10^{\prime}$ & $74^{\circ} 39^{\prime}$ & & v & 1990 & D. Silva & Etiqueta & $\begin{array}{l}\text { MHN-UNMSM/Smith- } \\
\text { sonian Inst. }\end{array}$ & Gonyleptoidea & Cosmetidae & A. Ayala-Apaza, 2021 \\
\hline 213 & MUSM-ENT 0512578 & 1 & Taito rorschachi Kury \& Barros, 2014* & Requena & Jenaro Herrera & $\begin{array}{l}\text { Centro de Investigación } \\
\text { jenaro Herrera,e pa pal- } \\
\text { meras Astrocarium sp. }\end{array}$ & 100 & $04^{\circ} 55^{\prime}$ & $73^{\circ} 45^{\prime}$ & 23 & viii & 1988 & D. Silva & Etiqueta & MHN-UNMSM & Gonyleptoidea & Cosmetidae & A. Ayala-Apaza, 2021 \\
\hline 214 & MUSM-ENT 0512601 & 5 & Taito rorschachi Kury \& Barros, 2014* & Loreto & Rio Samiria & Cocha Shinguito & 90 & $5^{\circ} 10^{\prime}$ & $74^{\circ} 39^{\prime}$ & & $i v-v$ & 1990 & T. Erwin & Etiqueta & $\begin{array}{l}\text { MHN-UNMSM/Smith- } \\
\text { sonian Inst. }\end{array}$ & Gonyleptoidea & Cosmetidae & A. Ayala-Apaza, 2021 \\
\hline 215 & MUSM-ENT 0514091 & 3 & Taito rorschachi Kury \& Barros, 2014* & Loreto & Rio Samiria & Cocha Shinguito & 90 & $5^{\circ} 10^{\prime}$ & $74^{\circ} 39^{\prime}$ & 12 & $\mathrm{v}$ & 1990 & T. Erwin & Etiqueta & $\begin{array}{l}\text { MHN-UNMSM/Smith- } \\
\text { sonian Inst. }\end{array}$ & Gonyleptoidea & Cosmetidae & M. Medrano, 2021 \\
\hline 216 & MUSM-ENT 0514103 & 5 & Taito rorschachi Kury \& Barros, 2014* & Loreto & Rio Samiria & Cocha Shinguito & 90 & $5^{\circ} 10^{\prime}$ & $74^{\circ} 39^{\prime}$ & 30 & v & 1990 & T. Erwin & Etiqueta & $\begin{array}{l}\text { MHN-UNMSM/Smith- } \\
\text { sonian Inst. }\end{array}$ & Gonyleptoidea & Cosmetidae & A. Ayala-Apaza, 2021 \\
\hline 217 & MUSM-ENT 0514108 & 1 & Taito rorschachi Kury \& Barros, 2014* & Loreto & Rio Samiria & Cocha Shinguito & 90 & $5^{\circ} 10^{\prime}$ & $74^{\circ} 39^{\prime}$ & 30 & v & 1990 & D. Silva & Etiqueta & $\begin{array}{l}\text { MHN-UNMSM/Smith- } \\
\text { sonian Inst. }\end{array}$ & Gonyleptoidea & Cosmetidae & A. Ayala-Apaza, 2021 \\
\hline 218 & MUSM-ENT 0514111 & 3 & Taito rorschachi Kury \& Barros, 2014* & Loreto & Rio Samiria & Cocha Shinguito & 90 & $5^{\circ} 10^{\prime}$ & $74^{\circ} 39^{\prime}$ & & & & T. Erwin et al. & Etiqueta & $\begin{array}{l}\text { MHN-UNMSM/Smith- } \\
\text { sonian Inst. }\end{array}$ & Gonyleptoidea & Cosmetidae & A. Ayala-Apaza, 2021 \\
\hline 219 & MUSM-ENT 0514507 & 1 & Taito rorschachi Kury \& Barros, 2014* & Loreto & Rio Samiria & Cocha Shinguito & 90 & $5^{\circ} 10^{\prime}$ & $74^{\circ} 39^{\prime}$ & 30 & v & 1990 & D. Silva & Etiqueta & $\begin{array}{l}\text { MHN-UNMSM/Smith- } \\
\text { sonian Inst. }\end{array}$ & Gonyleptoidea & Cosmetidae & A. Ayala-Apaza, 2021 \\
\hline 220 & MUSM-ENT 0514509 & 1 & Taito rorschachi Kury \& Barros, 2014* & Requena & Jenaro Herrera & $\begin{array}{l}\text { Maniti, en palmeras } \\
\text { Astrocarium sp. }\end{array}$ & & $04^{\circ} 55^{\prime}$ & $73^{\circ} 45^{\prime}$ & 23 & $x$ & 1988 & I. Llosa & Google Earth & $\begin{array}{l}\text { ORSTOM/IIAP, ORS- } \\
\text { TOM/MHN-UNMSM }\end{array}$ & Gonyleptoidea & Cosmetidae & A. Ayala-Apaza, 2021 \\
\hline 221 & MUSM-ENT 0512199 & 1 & Taito sp.n. & Loreto & Rio Samiria & Cocha Shinguito & 90 & $5^{\circ} 10^{\prime}$ & $74^{\circ} 39^{\prime}$ & & v & 1990 & T. Erwin & Etiqueta & $\begin{array}{l}\text { MHN-UNMSM/Smith- } \\
\text { sonian Inst. }\end{array}$ & Gonyleptoidea & Cosmetidae & $\begin{array}{l}\text { A. Ayala-Apaza y M. Medrano, } \\
2021\end{array}$ \\
\hline 222 & MUSM-ENT 0512365 & 1 & Taito sp.n. & Loreto & Rio Samiria & Cocha Shinguito & 90 & $5^{\circ} 10^{\prime}$ & $74^{\circ} 39^{\prime}$ & & v & 1990 & D. Silva & Etiqueta & $\begin{array}{l}\text { MHN-UNMSM/Smith- } \\
\text { sonian Inst. }\end{array}$ & Gonyleptoidea & Cosmetidae & A. Ayala-Apaza, 2021 \\
\hline 223 & MUSM-ENT 0512562 & 3 & Taito sp.n. & Loreto & Rio Samiria & Cocha Shinguito & 90 & $5^{\circ} 10^{\prime}$ & $74^{\circ} 39^{\prime}$ & 17 & v & 1990 & & Etiqueta & $\begin{array}{l}\text { MHN-UNMSM/Smith- } \\
\text { sonian Inst. }\end{array}$ & Gonyleptoidea & Cosmetidae & A. Ayala-Apaza, 2021 \\
\hline 224 & MUSM-ENT 0514089 & 5 & Taito sp.n. & Loreto & Rio Samiria & Cocha Shinguito & 90 & $5^{\circ} 10^{\prime}$ & $74^{\circ} 39^{\prime}$ & 20 & $v$ & 1990 & D. Silva & Etiqueta & $\begin{array}{l}\text { MHN-UNMSM/Smith- } \\
\text { sonian Inst. }\end{array}$ & Gonyleptoidea & Cosmetidae & A. Ayala-Apaza, 2021 \\
\hline 225 & MUSM-ENT 0514093 & 2 & Taito sp.n. & Loreto & Rio Samiria & Cocha Shinguito & 90 & $5^{\circ} 10^{\prime}$ & $74^{\circ} 39^{\prime}$ & 23 & v & 1990 & T. Erwin & Etiqueta & $\begin{array}{l}\text { MHN-UNMSM/Smith- } \\
\text { sonian Inst. }\end{array}$ & Gonyleptoidea & Cosmetidae & M. Hara y A. Ayala-Apaza, 2021 \\
\hline 226 & MUSM-ENT 0514499 & 1 & Taito sp.n. & Loreto & Rio Samiria & Cocha Shinguito & 90 & $5^{\circ} 10^{\prime}$ & $74^{\circ} 39^{\prime}$ & & vi & 1990 & T. Erwin & Etiqueta & $\begin{array}{l}\text { MHN-UNMSM/Smith- } \\
\text { sonian Inst. }\end{array}$ & Gonyleptoidea & Cosmetidae & M. Hara y A. Ayala-Apaza, 2021 \\
\hline 227 & MUSM-ENT 0501237 & 1 & Thaumatocranaus sp.n. & Maynas & Río Napo & Rio Napo, terraza baja & & ${ }^{\circ} 51^{\prime} 43.2^{\prime \prime}$ & $75^{\circ} 24^{\prime} 07.2^{\prime \prime}$ & $16-18$ & ii & 2008 & W. Paredes & Etiqueta & Consultoría EIA & Gonyleptoidea & Gonyleptidae & M. Hara y A. Ayala-Apaza, 2021 \\
\hline 228 & MUSM-ENT 0512181 & 3 & Thaumatocranaus sp.n. & Maynas & Río Curaray & & 216 & $1^{\circ} 35^{\prime} 16.1^{\prime \prime}$ & $75^{\circ} 24^{\prime} 19.7^{\prime \prime}$ & 29 & ii & 2008 & W. Paredes & Etiqueta & Consultoría EIA & Gonyleptoidea & Gonyleptidae & A. Ayala-Apaza, 2021 \\
\hline 229 & MUSM-ENT 0512313 & 2 & Thaumatocranaus sp.n. & Maynas & Río Napo & & 190 & $0^{\circ} 58^{\prime} 48.80^{\prime \prime}$ & $75^{\circ} 13^{\prime 2} 27.69 "$ & 11 & $\mathrm{i}$ & 2009 & R. Yawarcani & Etiqueta & Consultoría EIA & Gonyleptoidea & Gonyleptidae & A. Ayala-Apaza, 2021 \\
\hline 230 & MUSM-ENT 0501260 & 2 & Ventripila marginata Roewer, $1917^{*}$ & Maynas & Río Napo & & 210 & $1^{\circ} 52^{\prime} 13.13$ & $74^{\circ} 47^{\prime} 25.59 "$ & 11 & $\mathrm{i}$ & 2009 & W. Yawarcani & Etiqueta & Consultoría EIA & Gonyleptoidea & Cranaidae & A. Ayala-Apaza, 2021 \\
\hline 231 & MUSM-ENT 0511968 & 5 & Ventripila marginata Roewer, $1917^{*}$ & Maynas & Río Napo & & 210 & $1^{\circ} 45^{\prime} 23.44^{\prime \prime}$ & $74^{\circ} 43^{\prime} 22.93^{\prime \prime}$ & 22 & xii & 2008 & W. Yawarcani & Etiqueta & Consultoría EIA & Gonyleptoidea & Cranaidae & A. Ayala-Apaza, 2021 \\
\hline 232 & MUSM-ENT 0511975 & 1 & Ventripila marginata Roewer, $1917^{*}$ & Loreto & Rio Samiria & Cocha Shinguito & 90 & $5^{\circ} 10^{\prime}$ & $74^{\circ} 39^{\prime}$ & 14 & v & 1990 & T. Erwin & Etiqueta & $\begin{array}{l}\text { MHN-UNMSM/Smith- } \\
\text { sonian Inst. }\end{array}$ & Gonyleptoidea & Cranaidae & A. Ayala-Apaza, 2021 \\
\hline 233 & MUSM-ENT 0511982 & 1 & Ventripila marginata Roewer, $1917^{*}$ & Maynas & Río Curaray & & 196 & $1^{\circ} 31^{\prime \prime 46.2 " ~}$ & $75^{\circ} 25^{\prime} 30.4^{\prime \prime}$ & 2 & iii & 2008 & W. Yawarcani & Etiqueta & Consultoría EIA & Gonyleptoidea & Cranaidae & $\begin{array}{l}\text { A. Ayala-Apaza y A. Pérez-Gonzá } \\
\text { lez, 2020 }\end{array}$ \\
\hline 234 & MUSM-ENT 0513079 & 1 & Ventripila marginata Roewer, $1917^{*}$ & Maynas & Rio Curaray & & 204 & $1956 ' 5.7 "$ & $75^{\circ} 22^{\prime} 20.8^{\prime \prime}$ & 10 & ii & 2008 & W. Paredes & Etiqueta & Consultoría EIA & Gonyleptoidea & Cranaidae & $\begin{array}{l}\text { A. Pérez-González y A. Ayala- } \\
\text { Apaza, } 2020\end{array}$ \\
\hline 235 & MUSM-ENT 0511803 & 1 & Zalmoxidae 1 & Loreto & Rio Samiria & Cocha Shinguito & 90 & $5^{\circ} 10^{\prime}$ & $74^{\circ} 39^{\prime}$ & 24 & $\mathrm{v}$ & 1990 & T. Erwin & Etiqueta & $\begin{array}{l}\text { MHN-UNMSM/Smith- } \\
\text { sonian Inst. }\end{array}$ & Zalmoxoidea & Zalmoxidae & $\begin{array}{l}\text { A. Ayala-Apaza y A. Pérez-Gonzá } \\
\text { lez, } 2020\end{array}$ \\
\hline 236 & MUSM-ENT 0511805 & 3 & Zalmoxidae 1 & Loreto & Rio Samiria & Cocha Shinguito & 90 & $5^{\circ} 10^{\prime}$ & $74^{\circ} 39^{\prime}$ & 15 & v & 1990 & D. Silva & Etiqueta & $\begin{array}{l}\text { MHN-UNMSM/Smith- } \\
\text { sonian Inst. }\end{array}$ & Zalmoxoidea & Zalmoxidae & $\begin{array}{l}\text { A. Pérez-González y A. Ayala- } \\
\text { Apaza, 2020 }\end{array}$ \\
\hline 237 & MUSM-ENT 0511990 & 3 & Zalmoxidae 1 & Loreto & Rio Samiria & Cocha Shinguito & 90 & $5^{\circ} 10^{\prime}$ & $74^{\circ} 39^{\prime}$ & 16 & v & 1990 & T. Erwin & Etiqueta & $\begin{array}{l}\text { MHN-UNMSM/Smith- } \\
\text { sonian Inst. }\end{array}$ & Zalmoxoidea & Zalmoxidae & $\begin{array}{l}\text { A. Ayala-Apaza y A. Pérez-Gonzá } \\
\text { lez, } 2020\end{array}$ \\
\hline 238 & MUSM-ENT 0511995 & 1 & Zalmoxidae 1 & Loreto & Río Samiria & Cocha Shinguito & 90 & $5^{\circ} 10^{\prime}$ & $74^{4} 39^{\prime}$ & 22 & v & 1990 & T. Erwin & Etiqueta & NLRP/Carabidae & Zalmoxoidea & Zalmoxidae & $\begin{array}{l}\text { A. Pérez-González y A. Ayala- } \\
\text { Apaza, } 2020\end{array}$ \\
\hline
\end{tabular}


LOS LANIATORES (ARACHNIDA: OpILONES: GRASSATORES) DEL DEPARTAMENTO DE LoREto

\begin{tabular}{|c|c|c|c|c|c|c|c|c|c|c|c|c|c|c|c|c|c|c|}
\hline \# & Código de ingreso & No. Ind. & Especie/Morfoespecie & Provincia & Localidad & Lugar de colecta & Altituc & datitud & Longitud & Dia & Mes & Año & colectores & $\begin{array}{l}\text { Fuente para } \\
\text { georeferen- } \\
\text { ciación }\end{array}$ & Proyecto de colecta & Superfamilia & Familia & Identificación \\
\hline 239 & MUSM-ENT 0512490 & 53 & Zalmoxidae 1 & Loreto & Río Samiria & Cocha Shinguito & 90 & $5^{\circ} 10^{\prime}$ & $74^{\circ} 39^{\prime}$ & 16 & v & 1990 & T. Erwin & Etiqueta & $\begin{array}{l}\text { MHN-UNMSM/Smith- } \\
\text { sonian Inst. }\end{array}$ & Zalmoxoidea & Zalmoxidae & $\begin{array}{l}\text { A. Ayala-Apaza y A. Pérez-Gonzá } \\
\text { lez, } 2020\end{array}$ \\
\hline 240 & MUSM-ENT 0512491 & 15 & Zalmoxidae 1 & Loreto & Río Samiria & Cocha Shinguito & 90 & $5^{\circ} 10^{\prime}$ & $74^{\circ} 39^{\prime}$ & 16 & v & 1990 & T. Erwin & Etiqueta & $\begin{array}{l}\text { MHN-UNMSM/Smith- } \\
\text { sonian Inst. }\end{array}$ & Zalmoxoidea & Zalmoxidae & $\begin{array}{l}\text { A. Pérez-González y A. Ayala- } \\
\text { Apaza, } 2020\end{array}$ \\
\hline 241 & MUSM-ENT 0512523 & 1 & Zalmoxidae 1 & Loreto & Río Samiria & Cocha Shinguito & 90 & $5^{\circ} 10^{\prime}$ & $74^{\circ} 39^{\prime}$ & 24 & $\mathrm{v}$ & 1990 & T. Erwin & Etiqueta & $\begin{array}{l}\text { MHN-UNMSM/Smith- } \\
\text { sonian Inst. }\end{array}$ & Zalmoxoidea & Zalmoxidae & $\begin{array}{l}\text { A. Ayala-Apaza y A. Pérez-Gonzá } \\
\text { lez, } 2020\end{array}$ \\
\hline 242 & MUSM-ENT 0512602 & 26 & Zalmoxidae 1 & Loreto & Río Samiria & Cocha Shinguito & 90 & $5^{\circ} 10^{\prime}$ & $74^{\circ} 39^{\prime}$ & 24 & vi & 1990 & T. Erwin & Etiqueta & $\begin{array}{l}\text { MHN-UNMSM/Smith- } \\
\text { sonian Inst. }\end{array}$ & Zalmoxoidea & Zalmoxidae & $\begin{array}{l}\text { A. Pérez-Gonzzález y A. Ayala- } \\
\text { Apaza, } 2020\end{array}$ \\
\hline 243 & MUSM-ENT 0512778 & 9 & Zalmoxidae 1 & Loreto & Río Samiria & Cocha Shinguito & 90 & $5^{\circ} 10^{\prime}$ & $74^{\circ} 39^{\prime}$ & 13 & $\mathrm{v}$ & 1990 & T. Erwin & Etiqueta & $\begin{array}{l}\text { MHN-UNMSM/Smith- } \\
\text { sonian Inst. }\end{array}$ & Zalmoxoidea & Zalmoxidae & $\begin{array}{l}\text { A. Ayala-Apaza y A. Pérez-Gonzá } \\
\text { lez, 2020 }\end{array}$ \\
\hline 244 & MUSM-ENT 0512833 & 2 & Zalmoxidae 1 & Loreto & Río Samiria & Cocha Shinguito & 90 & $5^{\circ} 10^{\prime}$ & $74^{\circ} 39^{\prime}$ & & v-vi & 1990 & T. Erwin & Etiqueta & $\begin{array}{l}\text { MHN-UNMSM/Smith- } \\
\text { sonian Inst. }\end{array}$ & Zalmoxoidea & Zalmoxidae & $\begin{array}{l}\text { A. Pérez-González y A. Ayala- } \\
\text { Appza, } 2020\end{array}$ \\
\hline 245 & MUSM-ENT 0514168 & 1 & Zalmoxidae 1 & Loreto & Río Samiria & Cocha Shinguito & 90 & $5^{\circ} 10^{\prime}$ & $74^{\circ} 39^{\prime}$ & 23 & v & 1990 & T. Erwin & Etiqueta & $\begin{array}{l}\text { MHN-UNMSM/Smith- } \\
\text { sonian Inst. }\end{array}$ & Zalmoxoidea & Zalmoxidae & $\begin{array}{l}\text { A. Ayala-Apaza y A. Pérez-Gonzá } \\
\text { lez, } 2020\end{array}$ \\
\hline 246 & MUSM-ENT 0514169 & 2 & Zalmoxidae 1 & Loreto & Río Samiria & Cocha Shinguito & 90 & $5^{\circ} 10^{\prime}$ & $74^{\circ} 39^{\prime}$ & 23 & v & 1990 & T. Erwin & Etiqueta & $\begin{array}{l}\text { MHN-UNMSM/Smith- } \\
\text { sonian IIst. }\end{array}$ & Zalmoxoidea & Zalmoxidae & $\begin{array}{l}\text { A. Pérez-González y A. Ayala- } \\
\text { Apaza, } 2020\end{array}$ \\
\hline 247 & MUSM-ENT 0514175 & 1 & Zalmoxidae 1 & Loreto & Río Samiria & Cocha Shinguito & 90 & $5^{\circ} 10^{\prime}$ & $74^{\circ} 39^{\prime}$ & 23 & v & 1990 & T. Erwin & Etiqueta & NLRP/Carabidae & Zalmoxoidea & Zalmoxidae & $\begin{array}{l}\text { A. Ayala-Apaza y A. Pérez-Gonzá } \\
\text { lez, } 2020\end{array}$ \\
\hline 248 & MUSM-ENT 0514177 & 1 & Zalmoxidae 1 & Loreto & Río Samiria & Cocha Shinguito & 90 & $5^{\circ} 10^{\prime}$ & $74^{\circ} 39^{\prime}$ & 30 & v & 1990 & T. Erwin & Etiqueta & $\begin{array}{l}\text { MHN-UNMSM/Smith- } \\
\text { sonian Inst. }\end{array}$ & Zalmoxoidea & Zalmoxidae & $\begin{array}{l}\text { A. Pérez-González y A. Ayala- } \\
\text { Apaza, } 2020\end{array}$ \\
\hline 249 & MUSM-ENT 0514178 & 6 & Zalmoxidae 1 & Loreto & Río Samiria & Cocha Shinguito & 90 & $5^{\circ} 10^{\prime}$ & $74^{\circ} 39^{\prime}$ & 30 & v & 1990 & T. Erwin & Etiqueta & $\begin{array}{l}\text { MHN-UNMSM/Smith- } \\
\text { sonian Inst. }\end{array}$ & Zalmoxoidea & Zalmoxidae & $\begin{array}{l}\text { A. Ayala-Apaza y A. Pérez-Gonzá } \\
\text { lez, } 2020\end{array}$ \\
\hline 250 & MUSM-ENT 0514180 & 1 & Zalmoxidae 1 & Loreto & Río Samiria & Cocha Shinguito & 90 & $5^{\circ} 10^{\prime}$ & $74^{\circ} 39^{\prime}$ & & $\mathrm{v}$ & 1990 & D. Silva & Etiqueta & $\begin{array}{l}\text { MHN-UNMSM/Smith- } \\
\text { sonian Inst. }\end{array}$ & Zalmoxoidea & Zalmoxidae & $\begin{array}{l}\text { A. Pérez-González y A. Ayala- } \\
\text { Apaza, } 2020\end{array}$ \\
\hline 251 & MUSM-ENT 0514511 & 1 & Zalmoxidae 1 & Loreto & Río Samiria & Cocha Shinguito & 90 & $5^{\circ} 10^{\prime}$ & $74^{\circ} 39^{\prime}$ & 16 & v & 1990 & T. Erwin & Etiqueta & $\begin{array}{l}\text { MHN-UNMSM/Smith- } \\
\text { sonian Inst. }\end{array}$ & Zalmoxoidea & Zalmoxidae & $\begin{array}{l}\text { A. Ayala-Apaza y A. Pérez-Gonzá } \\
\text { lez, } 2020\end{array}$ \\
\hline 252 & MUSM-ENT 0511801 & 2 & Zalmoxidae 2 & Loreto & Río Samiria & Cocha Shinguito & 90 & $5^{\circ} 10^{\prime}$ & $74^{\circ} 39^{\prime}$ & 23 & v & 1990 & T. Erwin & Etiqueta & $\begin{array}{l}\text { MHN-UNMSM/Smith- } \\
\text { sonian Inst. }\end{array}$ & Zalmoxoidea & Zalmoxidae & $\begin{array}{l}\text { A. Pérez-González y A. Ayala- } \\
\text { Apaza, } 2020\end{array}$ \\
\hline 253 & MUSM-ENT 0511800 & 1 & Zalmoxidae 3 & Maynas & Río Curaray & & & $1^{\circ} 42^{\prime \prime} 49.7^{\prime \prime}$ & $75^{\circ} 29^{\prime} 09.9^{\prime \prime}$ & 25 & ii & 2008 & w. Yawarcani & Etiqueta & Consultoría EIA & Zalmoxoidea & Zalmoxidae & $\begin{array}{l}\text { A. Ayala-Apaza y A. Pérez-Gonzá } \\
\text { lez, } 2020\end{array}$ \\
\hline 254 & MUSM-ENT 0511807 & 1 & Zalmoxidae 4 & Loreto & Río Samiria & Cocha Shinguito & 90 & $5^{\circ} 10^{\prime}$ & $74^{\circ} 39^{\prime}$ & 17 & v & 1990 & T. Erwin & Etiqueta & $\begin{array}{l}\text { MHN-UNMSM/Smith- } \\
\text { sonian Inst. }\end{array}$ & Zalmoxoidea & Zalmoxidae & $\begin{array}{l}\text { A. Pérez-González y A. Ayala- } \\
\text { Apaza, } 2020\end{array}$ \\
\hline 255 & MUSM-ENT 0511939 & 4 & Zalmoxidae 4 & Loreto & Río Samiria & Cocha Shinguito & 90 & $5^{\circ} 10^{\prime}$ & $74^{\circ} 39^{\prime}$ & & v & 1990 & T. Erwin & Etiqueta & $\begin{array}{l}\text { MHN-UNMSM/Smith- } \\
\text { sonian Inst. }\end{array}$ & Zalmoxoidea & Zalmoxidae & $\begin{array}{l}\text { A. Ayala-Apaza y A. Pérez-Gonzá } \\
\text { lez, } 2020 \text {. }\end{array}$ \\
\hline 256 & MUSM-ENT 0511989 & 3 & Zalmoxidae 4 & Loreto & Río Samiria & Cocha Shinguito & 90 & $5^{\circ} 10^{\prime}$ & $74^{\circ} 39^{\prime}$ & 23 & v & 1990 & T. Erwin & Etiqueta & NLRP/Carabidae & Zalmoxoidea & Zalmoxidae & $\begin{array}{l}\text { A. Pérez-González y A. Ayala- } \\
\text { Apaza, } 2020\end{array}$ \\
\hline 257 & MUSM-ENT 0512244 & 2 & Zalmoxidae 4 & Loreto & Río Samiria & Cocha Shinguito & 90 & $5^{\circ} 10^{\prime}$ & $74^{\circ} 39^{\prime}$ & 27 & v & 1990 & D. silva & Etiqueta & $\begin{array}{l}\text { MHN-UNMSM/Smith- } \\
\text { sonian Inst. }\end{array}$ & Zalmoxoidea & Zalmoxidae & $\begin{array}{l}\text { A. Ayala-Apaza y A. Pérez-Gonzá } \\
\text { lez, } 2020\end{array}$ \\
\hline 258 & MUSM-ENT 0512265 & 3 & Zalmoxidae 4 & Loreto & Río Samiria & Cocha Shinguito & 90 & $5^{\circ} 10^{\prime}$ & $74^{\circ} 39^{\prime}$ & & $v-v i$ & 1990 & & Etiqueta & $\begin{array}{l}\text { MHN-UNMSM/Smith- } \\
\text { sonian Inst. }\end{array}$ & Zalmoxoidea & Zalmoxidae & $\begin{array}{l}\text { A. Pérez-González y A. Ayala- } \\
\text { Apaza, } 2020\end{array}$ \\
\hline 259 & MUSM-ENT 0512834 & 3 & Zalmoxidae 4 & Loreto & Río Samiria & Cocha Shinguito & 90 & $5^{\circ} 10^{\prime}$ & $74^{\circ} 39^{\prime}$ & 27 & v & 1990 & T. Erwin & Etiqueta & $\begin{array}{l}\text { MHN-UNMSM/Smith- } \\
\text { sonian Inst. }\end{array}$ & Zalmoxoidea & Zalmoxidae & $\begin{array}{l}\text { A. Ayala-Apaza y A. Pérez-Gonzá } \\
\text { lez, } 2020\end{array}$ \\
\hline 260 & MUSM-ENT 0514173 & 2 & Zalmoxidae 4 & Loreto & Río Samiria & Cocha Shinguito & 90 & $5^{\circ} 10^{\prime}$ & $74^{\circ} 39^{\prime}$ & 16 & v & 1990 & T. Erwin & Etiqueta & $\begin{array}{l}\text { MHN-UNMSM/Smith- } \\
\text { sonian Inst. }\end{array}$ & Zalmoxoidea & Zalmoxidae & $\begin{array}{l}\text { A. Ayala-Apaza y A. Pérez-Gonzá } \\
\text { lez, } 2020\end{array}$ \\
\hline 261 & MUSM-ENT 0514179 & 2 & Zalmoxidae 4 & Loreto & Río Samiria & Cocha Shinguito & 90 & $5^{\circ} 10^{\prime}$ & $74^{\circ} 39^{\prime}$ & 30 & v & 1990 & T. Erwin & Etiqueta & $\begin{array}{l}\text { MHN-UNMSM/Smith- } \\
\text { sonian Inst. }\end{array}$ & Zalmoxoidea & Zalmoxidae & A. Ayala-Apaza, 2021 \\
\hline 262 & MUSM-ENT 0514181 & 1 & Zalmoxidae 4 & Loreto & Río Samiria & Cocha Shinguito & 90 & $5^{\circ} 10^{\prime}$ & $74^{\circ} 39^{\prime}$ & 20 & $\mathrm{v}$ & 1990 & & Etiqueta & $\begin{array}{l}\text { MHN-UNMSM/Smith- } \\
\text { sonian Inst. }\end{array}$ & Zalmoxoidea & Zalmoxidae & $\begin{array}{l}\text { O. Villarreal-Manzanilla y A. } \\
\text { Ayala, } 2021\end{array}$ \\
\hline
\end{tabular}



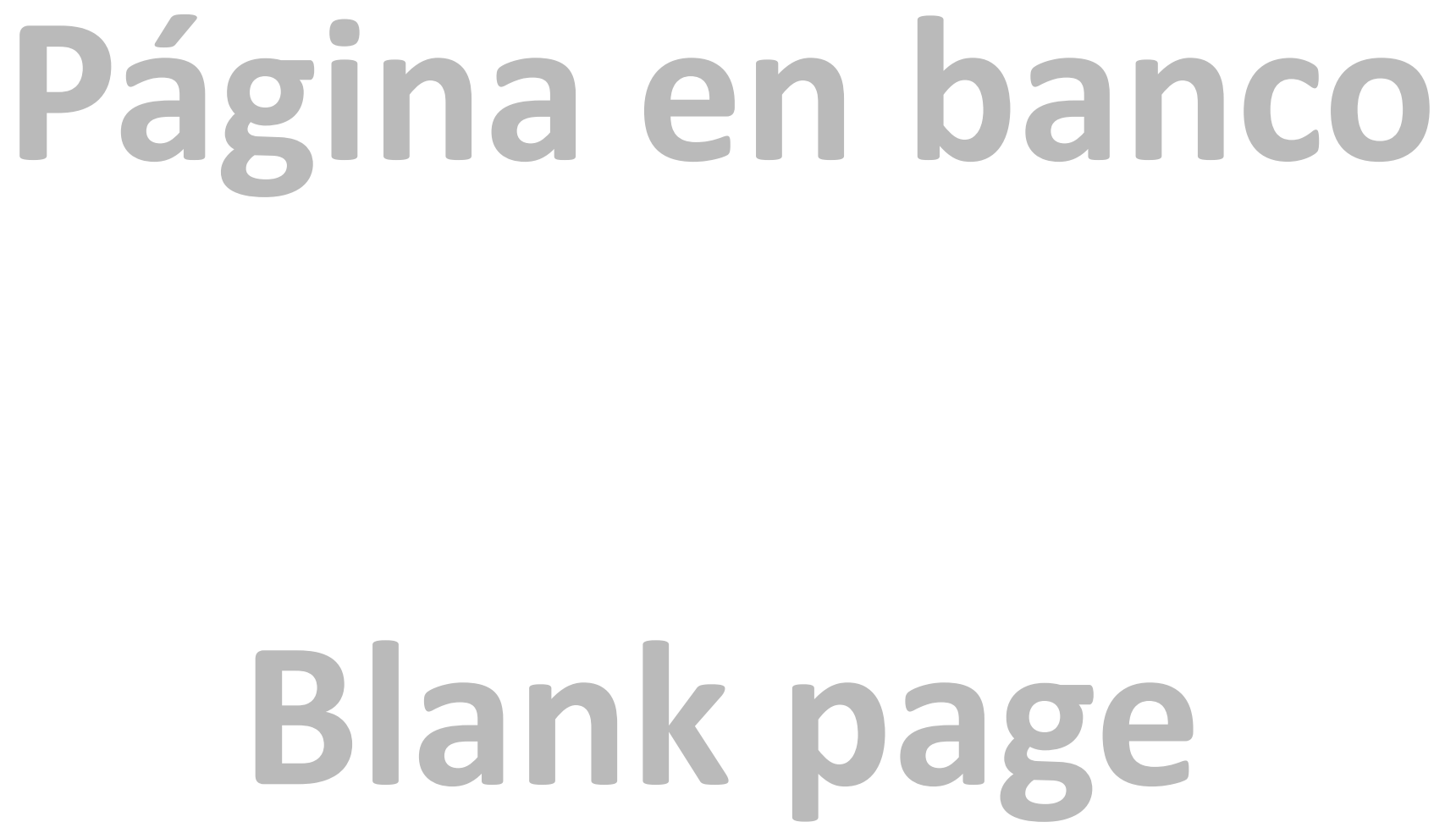